\title{
Quantitative EEG and machine learning methods for the detection of epileptic seizures and cerebral asymmetry
}

Citation for published version (APA):

Bogaarts, J. G. (2017). Quantitative EEG and machine learning methods for the detection of epileptic seizures and cerebral asymmetry. [Doctoral Thesis, Maastricht University]. Maastricht University. https://doi.org/10.26481/dis.20170223jgb

Document status and date:

Published: 01/01/2017

DOI:

10.26481/dis.20170223jgb

Document Version:

Publisher's PDF, also known as Version of record

Please check the document version of this publication:

- A submitted manuscript is the version of the article upon submission and before peer-review. There can be important differences between the submitted version and the official published version of record.

People interested in the research are advised to contact the author for the final version of the publication, or visit the DOI to the publisher's website.

- The final author version and the galley proof are versions of the publication after peer review.

- The final published version features the final layout of the paper including the volume, issue and page numbers.

Link to publication

\footnotetext{
General rights rights.

- You may freely distribute the URL identifying the publication in the public portal. please follow below link for the End User Agreement:

www.umlib.nl/taverne-license

Take down policy

If you believe that this document breaches copyright please contact us at:

repository@maastrichtuniversity.nl

providing details and we will investigate your claim.
}

Copyright and moral rights for the publications made accessible in the public portal are retained by the authors and/or other copyright owners and it is a condition of accessing publications that users recognise and abide by the legal requirements associated with these

- Users may download and print one copy of any publication from the public portal for the purpose of private study or research.

- You may not further distribute the material or use it for any profit-making activity or commercial gain

If the publication is distributed under the terms of Article 25fa of the Dutch Copyright Act, indicated by the "Taverne" license above, 
Quantitative EEG and machine learning methods for the detection of epileptic seizures and cerebral asymmetry 
ISBN: 978-94-6299-540-6

Copyright: (C 2017, Guy Bogaarts

Printed by: Ridderprint

Cover illustration: Marc Dekkers 
Quantitative EEG and machine learning methods for the detection of epileptic seizures and cerebral asymmetry

\section{PROEFSCHRIFT}

ter verkrijging van de graad van doctor

aan de Universiteit Maastricht,

op gezag van de Rector Magnificus,

Prof. dr. R.M. Letschert

volgens het besluit van het College van Decanen,

in het openbaar te verdedigen

op donderdag 23 februari 2017 om 10.00 uur

door

Jan Guy Bogaarts

Geboren op 2 april 1986 te Waalwijk 
Promotor:

Prof.dr. W.H. Mess

Copromotores:

Dr.ir. J.P.H. Reulen

Dr.ir. E.D. Gommer

Beoordelingscommissie

Prof.dr. H.W.M. Steinbusch (voorzitter)

Prof.dr. ir. S.J.A. van Huffel (Katholieke Universiteit Leuven, België)

Prof.dr. ir. M.J.A.M. van Putten (Universiteit Twente)

Dr. W.N.K.A. van Mook

Dr. M.C.G. Vlooswijk 


\section{Contents}

Chapter 1

Chapter 2

Chapter 3

Chapter 4

Chapter 5

Chapter 6

Chapter 7

Chapter 8

References

Summary

Samenvatting
Introduction

EEG feature pre-processing for neonatal epileptic seizure detection

SVM based, age-independent, automated epileptic seizure detection combining dynamic feature normalization with EEG novelty detection

From non-patient towards patient specific 87 epileptic seizure detection in comatose patients

An improved qEEG index for asymmetry 105 detection during the Wada test

General Discussion

Valorization 
Dankwoord

$\begin{array}{ll}\text { Publications } & 163\end{array}$

$\begin{array}{ll}\text { Curriculum Vitae } & 164\end{array}$ 
Chapter 1

Introduction 


\section{Chapter 1}

Brain damage can cause many types of brain dysfunction ranging from impairment of specific functions, to complete loss of consciousness or to the occurrence of epileptic seizures. Brain dysfunction can be caused by metabolic abnormalities such as low blood oxygen levels (hypoxia) or low levels of blood sugar (hypoglycemia), insufficient blood supply to the brain, head injuries, brain tumors or abscesses, and epileptic disorders. On an intensive care unit (ICU) all vital parameters are monitored, such as ElectroCardioGram (ECG), heart rate, blood pressure, respiration, and blood oxygen saturation. Although brain dysfunction is often the primary reason for admittance to the ICU, brain function is only incidentally measured despite that an appropriate modality for doing so is available. Scalp Electroencephalography (EEG) is one of the most commonly used non-invasive ways of measuring cortical brain activity. EEG registrations, lasting typically 20 to 30 minutes, are the standard procedure of assessing brain function. While these EEG registrations provide intermittent information on the functioning of the brain, continuous EEG (cEEG) measurements allow for real-time monitoring. Albeit that despite the EEG is recorded continuous, evaluation is often retrospective. The main reason for this is that the manual analysis of long term continuous EEG recordings is labor intensive, time consuming, requires specialized skills and is therefore also expensive. Automated EEG analysis is therefore much warranted. However, to become an established procedure on the ICU, automated analysis should be reliable enough to (partly) take over the manual analysis. In case of the detection of clinically relevant events this means a high sensitivity while simultaneously keeping the number of false detections at a minimum.

Among the most important phenomena to be seen in the EEG of patients with brain dysfunction are electrographic seizures. Seizures are classified as either 'convulsive' or 'non-convulsive'. In the first case the cortical epileptic activity is accompanied by clinical signs such as limb clonus or more subtle signs such as eye twitching, in the second case it is not. Such an electrographic or non-convulsive seizure (NCS) can only 
be depicted by means of an EEG. Although a distinction between clinical and subclinical seizures can be made on clinical grounds, no neurobiological basis for this distinction exists (1). Based on continuous EEG registrations of comatose patients, it is estimated that the incidence of seizures in patients with coma can be up to $48 \%$ (2). Of these comatose patient, $30 \%$ had non-convulsive seizures which means that the actual incidence of seizures is much higher than suggested by clinical suspicion alone (2).

As mentioned earlier, automated detection of seizures in the EEG could be a valuable tool to assist the human expert. On the one hand it allows for long-term continuous surveillance of brain function of ICU patients. On the other hand it could also be used in a more general way in every scenario where a human expert has to evaluate large amounts of EEG data. In such cases an algorithm could detect and summarize the most important findings and highlight those EEG segments requiring further inspection by a human expert. In the first case an algorithm is considered an alternative for a human expert while in the latter case it is a tool to assist a human expert. Considering the fact that the human expert is the gold standard and $100 \%$ accuracy is likely not achievable, considering an algorithm as a tool instead of an alternative to a human expert will facilitate the evaluation and acceptance of automated EEG analysis algorithms.

The remainder of this chapter is used to give an overview of general EEG properties, quantitative EEG (qEEG) measures and continuous ICU monitoring. Finally the outline of the thesis is described. 
Chapter 1

\section{General EEG properties}

The first human EEG recording dates back from 1924 and the principles of EEG recording have not changed ever since (3). The EEG refers to the signal that is obtained by registering the potential differences between electrodes placed on the scalp. Scalp EEG measures voltage fluctuations in the order of 10 to $100 \mu \mathrm{V}$ caused by ion currents generated by thousands of similarly orientated dendrites of pyramidal neurons (4). The temporal resolution of the EEG is very good (nowadays in de milliseconds range) although the spatial resolution is bad compared to other non-invasive methods (in the $\mathrm{cm}$ range), which is due to volume conduction of the cerebrospinal fluid, bone and other tissues causing an electrical spread of activity over neighboring electrodes (5). Nevertheless, the EEG plays an important role in diagnostics and monitoring because of its high time resolution, low costs and general availability of recording equipment $(1,6-8)$. Furthermore, apart from MEG, there are no other measurement modalities that measure neuronal activity as direct as EEG.

The $10-20$ system

The electrical cortical activity is usually recorded at equally distributed standardized locations (international 10-20 system) on the scalp (9). The electrode locations are defined by dividing the head into $10 \%$ and $20 \%$ angular intervals using the inion and nasion as landmarks (figure 1). The first letter of the electrode refers to the brain region it is placed on, and numbers refer to the left (odd) and right (even) hemisphere.

\section{Montages}

Measuring an EEG signal always involves a differential signal between two electrodes. In practice EEG signals are recorded using differential 
amplifiers where the voltage difference between two electrodes is amplified. To visualize EEG data, different montages can be constructed by creating different sets of constructed subtractions between different unipolar signals. In the following chapters the common average reference montage is used for subsequent analyses.
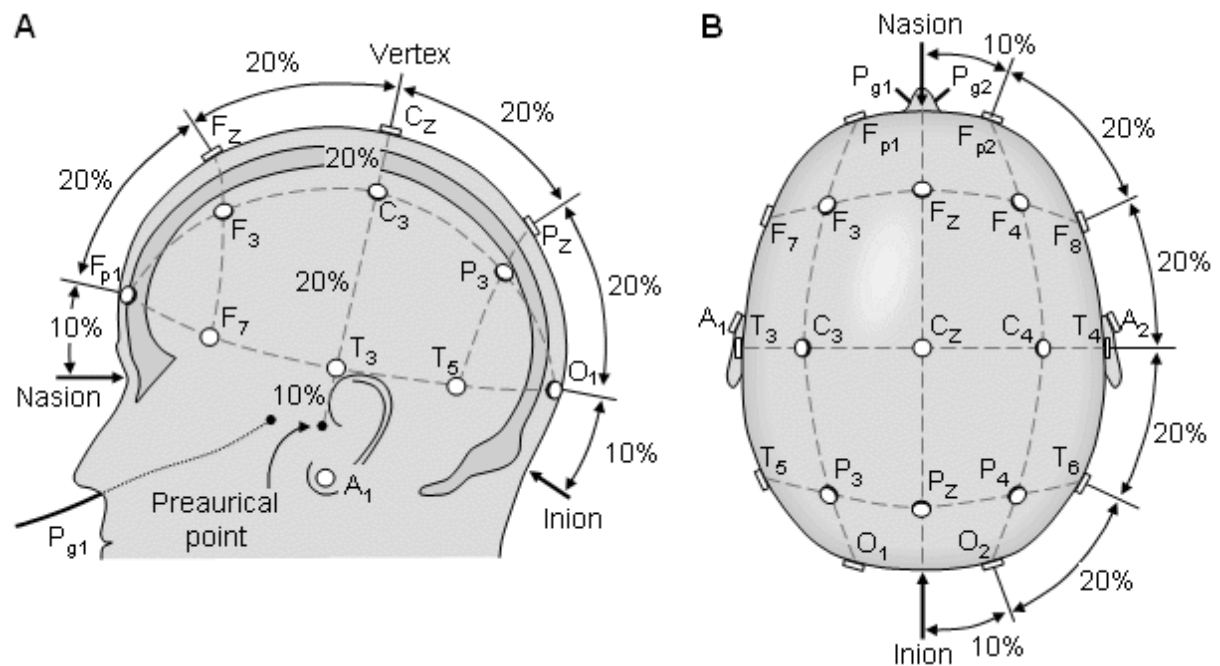

Figure 1 The international 10-20 system seen from (A) left and (B) above the head. $A=$ Ear lobe,$C=$ central, $P g=$ nasopharyngeal, $P=$ parietal, $F=$ frontal, $F p=$ frontal polar, $\mathrm{O}=$ occipital. From (10).

\section{Types of EEG activity}

General EEG properties can mainly be divided into background activity and transients $(4,5)$. Both these categories are evaluated for abnormalities, deviations from the norm, or deviations from what was expected for a specific patient. Changes in the hemispheric symmetry of the background activity and the occurrence of transients in the form of epileptic activity are especially relevant for neuro-monitoring purposes on the ICU. Both asymmetry and epileptic activity may be signs of underlying pathology that might require intervention. In the next section a short outline of general EEG properties is given. 


\section{Chapter 1}

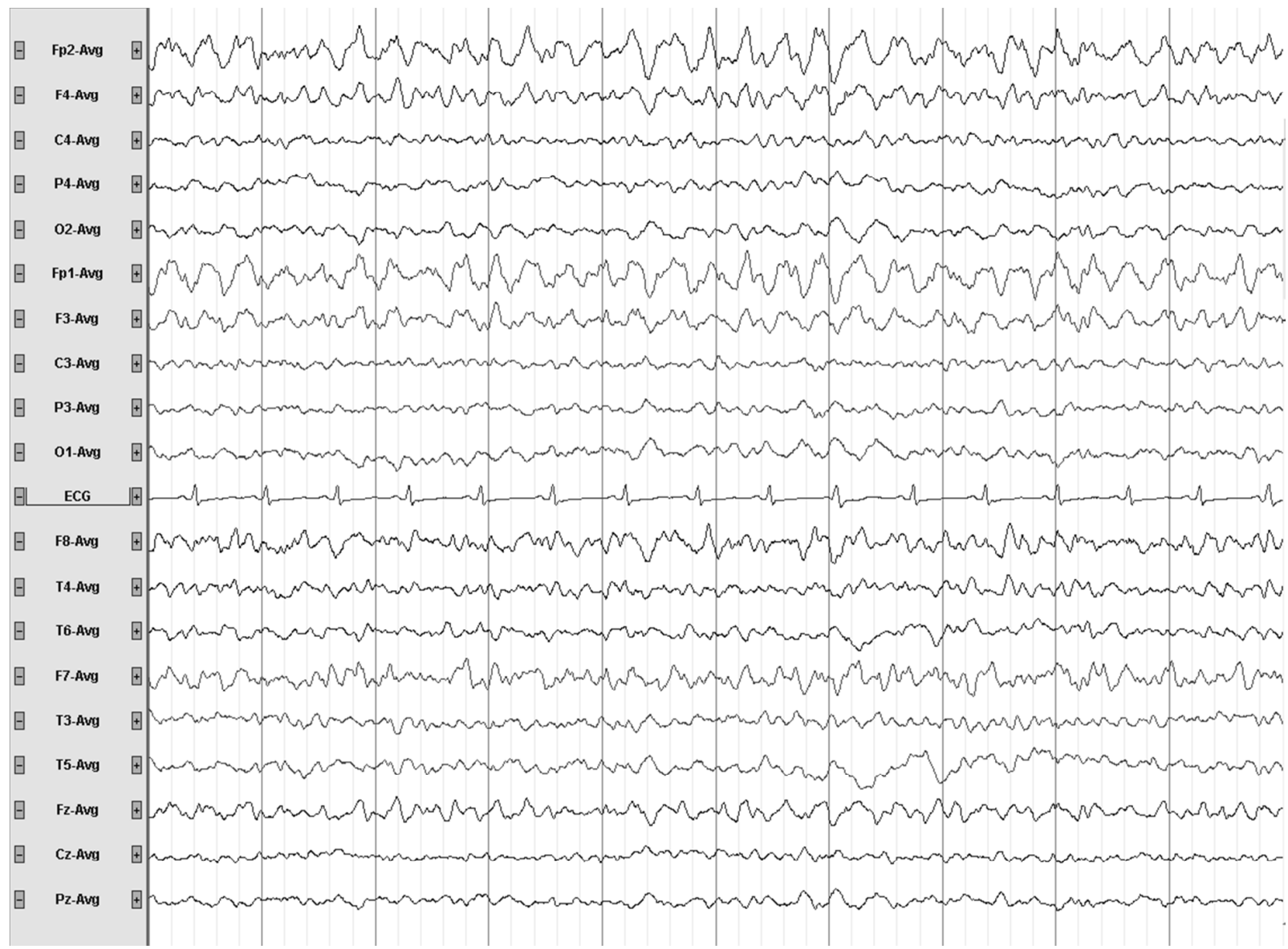

Figure 2 19-Channel scalp EEG recording in reference montage from a comatose patient. Channel FP1 and FP2 show bilateral frontal delta activity which develops into a non-convulsive seizure.

\section{Background EEG}

The background activity describes the general patterns that are observed in the EEG. These patterns are generally described as rhythms from a certain frequency range. In increasing order of frequency the following frequency bands are used: delta $(<4 \mathrm{~Hz})$, theta $(4-8 \mathrm{~Hz})$, alpha $(8-12 \mathrm{~Hz})$, beta $(12-30 \mathrm{~Hz})$ and gamma $(>30 \mathrm{~Hz})(4)$. Each frequency band is associated with well-known properties and deviations or abnormalities are mostly related to pathologies or brain damage. Normal background properties are also related to age and circadian rhythm. For example slow activity in the delta and theta range is normal 
in children but considered abnormal in adults. Apart from differences in normal background patterns related to age, the EEG also shows a posterior-anterior gradient in both frequency and amplitude. Posterior rhythms are dominated by lower frequency and higher amplitudes, while oscillations with higher frequencies and lower amplitudes are characteristic for anterior regions. A lack of faster activity or the absence of the posterior anterior gradient in adults can be observed in comatose patients or in patients with neurodegenerative disease (5, 11). Another marker for a healthy brain is a symmetric distribution of frequency and amplitude between left and right hemispheres. Asymmetries in frequency or amplitude are usually related to pathological conditions such as ischemia, trauma or space occupying lesions.

\section{Transients}

Transients refer to short and abrupt changes of the EEG that can be caused by both normal and abnormal processes in the brain. Examples of normal transients are vertex waves, K-complexes and sleep spindles as seen in normal sleep. Also uncommon but normal variants exist such as the mu rhythms (12). The most common forms of abnormal transients are inter-ictal epileptiform discharges (IEDs). These IEDs usually take the form of spikes, sharp waves, spike trains and spikewave discharges (4). IEDs may occur periodically and are often lateralized (LPDs) or generalized (GPDs). IEDs are associated with epileptic seizures and poor outcome (13). In comatose patients often the distinctness of periodic IEDs waxes and wanes and could develop into electrographic seizures. However, it is often quite difficult to distinguish GPDs from seizure activity which raises the question if the effect of both GPDs and electrographic seizures is the same for the patient (14-16) . 
Chapter 1

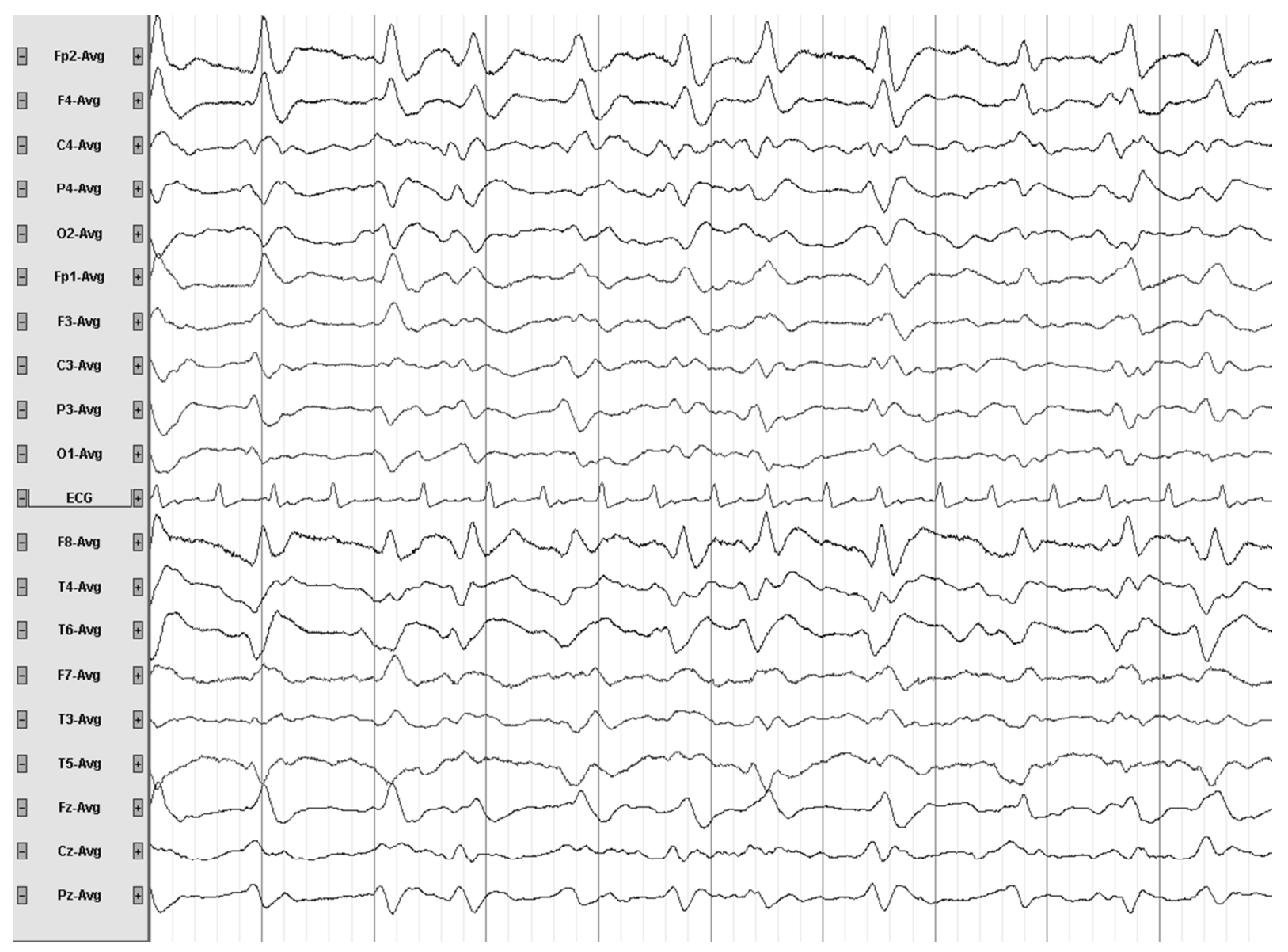

Figure 3 Example of periodic lateralized discharges (LPDs) originating from the right frontal region. The presence of LPDs is associated with the recurrence of seizures. In this patient these LPDs eventually developed into seizures.

Visual inspection of the EEG

Reviewing EEG recordings is a complex and time consuming task which can only be performed by experienced clinicians (4). The EEG is reviewed as multiple time series tiled horizontally, as illustrated in figure 2 and 3 . Using a variety of montages, a clinical neurophysiologist looks for abnormalities in background patterns and transient phenomena. Traditionally the EEG is reviewed visually in the time domain. Conventionally, the frequency content is band-pass filtered between 0.5 and $50 \mathrm{~Hz}$ although physiological and pathological EEG 
activity ranges at least from $0.01 \mathrm{~Hz}$ to several hundred $\mathrm{Hz}$ (17). Usually, EEG is viewed in windows of 10-30 seconds showing all channels simultaneously, while one scrolls through the recording. An EEG is usually described by a range of essential signal characteristics: frequency, amplitude, wave morphology, sustained activity, manner of occurrence (random, serial, continuous), localization, reactivity and interhemispheric differences (5). Technological advancements in the acquisition, storage and remote review of EEG facilitated the realization of continuous EEG monitoring (cEEG). Additionally quantitative EEG (qEEG) allows for a compressed view of hours of EEG and is helpful in various clinical applications including predicting or detecting epileptic seizures, classifying sleep stages, measuring depth of anesthesia, detection and monitoring of brain injury, and detecting abnormal brain states (18-22). For routine EEG registrations (duration approximately 20-30 minutes) and EEG registrations during a Wada (23) test or carotid endarterectomy (24), it is feasible and necessary to review the EEG in real-time. In case of cEEG however, the amount of data recorded is so overwhelming that visual inspection of all signals is just not feasible. So, the application of an automated analysis reproducing the visually made classifications as good as possible is mandatory if cEEG is to be implemented in daily clinical practice. Hence, quantitative EEG measures are developed to quantify the aforementioned EEG characteristics. These measures analyze different aspects of the signal in the time domain, such as number of zero crossings as well as the frequency domain, such as power in specific frequency bands, or e.g. entropy measures.

\section{ICU monitoring with cEEG}

Over the past two decades it has become clear that compared to routine EEG, cEEG significantly increases the sensitivity for detecting NCS and non-convulsive status epilepticus (NCSE) in critically ill patients 
Chapter 1

(6). The incidence of NCS or NCSE in this patient population ranges from 7 to $37 \%$ on all patients and up to $48 \%$ in patients with coma or prior convulsive status epilepticus (CSE) $(2,25,26)$. NCS or NCSE often lack obvious clinical signs which makes it difficult to detect at the bedside without EEG monitoring. The difficulties of detecting NCS or NCSE result in a delay or even lack of diagnosis $(27,28)$. With routine EEG only approximately $40-50 \%$ of patients with NCS or NCSE are detected (29, 30).

\section{Quantitative EEG features}

After digitization, an EEG signal can be represented as time series of raw data samples (31). Visual analysis of the raw EEG requires a considerable amount of neurophysiological knowledge and experience. As mentioned before, visual inspection during long-term monitoring is not always feasible and furthermore is subjective and does not easily allow for statistical analysis $(32,33)$. Therefore alternative methods have been introduced that enable the reader to inspect hours of EEG in a compressed form. These methods quantify information contained in an EEG signal (31) applying a variety of signal analysis approaches such as the Fourier transform, wavelet transform, chaos, entropy, time domain, empirical model decomposition, and speech recognition (3436). Some examples of qEEG features are: EEG power and normalized power per frequency band, signal variance, peak frequency of spectrum, spectral edge frequency, wavelet energy, curve length, zero crossings, kurtosis, non-linear energy, Shannon entropy and fisher information. Each of these qEEG features is calculated for each EEG channel separately. For displaying purposes they are often averaged per left and right hemisphere (figure 4). In addition to supplying the viewer with a compressed view of information contained in the EEG, qEEG features can also be used to classify EEG signals using mathematical functions. Each feature is designed to describe specific properties of the EEG signal. 


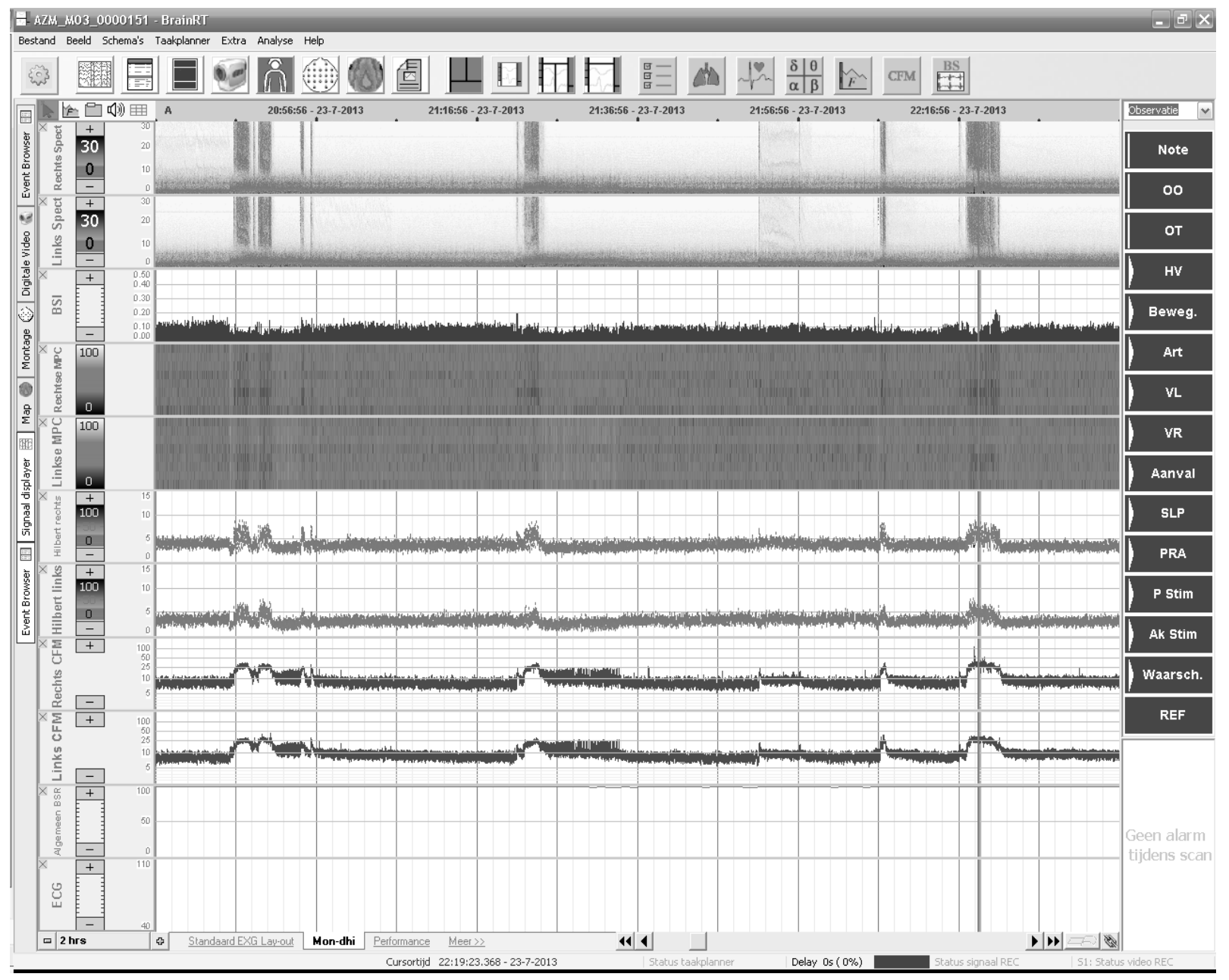

Figure 4 Example of 4 hours of EEG compressed into 5 different qEEG features. From top to bottom: spectral power from 0 to $30 \mathrm{~Hz}$ averaged over the right and left hemisphere; Brain symmetry index (BSI); Main phase coherence (MPC) for the right and left hemisphere; Hilbert transform per hemisphere; Cerebral function monitor (CFM) signal per hemisphere.

Feature based classification

In case feature values between two classes, for example seizure and non-seizure epochs, do not show any overlap, a single feature allows for a perfect classification. Unfortunately, in practice this is not often the case. This is because apart from being sensitive for the detection of seizures or asymmetry, qEEG features may also show similar values for artifacts or other rhythmic activity. As a result, epochs could be wrongly 


\section{Chapter 1}

classified. Furthermore, inter patient variability makes it even more difficult to correctly classify EEG epochs based on a single qEEG feature. Instead of using a single qEEG feature to classify EEG epochs, combining two or more qEEG features might exploit patterns in the data that allow for better discrimination then each feature separately. Exploiting patterns not readily visible in a dataset, by combining several features, lie at the heard of data driven classification techniques such as linear discriminant analysis (LDA), artificial neural networks, and support vector machines (SVM). When each epoch is described by $\mathrm{N}$ features, this can be seen as a vector representing a point in an $\mathrm{N}$-dimensional feature space where each feature is assigned to an axis and together span the multi-dimensional feature space. Where classification in one dimension translates to thresholding a single value, the threshold in multiple dimensions becomes a hyperplane. In case of a non-linearly separable classification task, a nonlinear mapping to a higher dimensional space (the number dimensions is limited to the number of data points) can be performed in which the data is linearly separable. This equates to a non-linear decision boundary in the original feature space.

Classification in statistics is the problem of identifying to which set of categories (class) a new observation belongs based on knowledge derived from a training set of observations of which the class is known. Classification is an example of the more general problem of pattern recognition which is the assignment of an output value to a given input value. The data is usually analyzed in the form of quantitative properties, the aforementioned features. An algorithm that implements classification is called a classifier. In the next section, two such classification methods, i.e. LDA and SVM that are used for classification in the following chapters of this thesis are described. Both methods are instances of supervised learning which means that they are trained using pre-classified (labeled) multi-dimensional training data. This labeling is usually performed by a human expert and serves as the gold standard. Training implies finding an optimal separation of two classes 
in this multidimensional feature space. In the following sections a feature vector is denoted as $\vec{x}$ and its class label y.

LDA is a method used in statistics, machine learning and patterns recognition to find a linear combination $\overrightarrow{\boldsymbol{w}}$ of features in $\overrightarrow{\boldsymbol{x}}$ which gives the best separation of classes (37). This linear combination can then be used as a linear classifier or as a dimensionality reduction for later classification. LDA is related to analysis of variance but has continuous independent variables and categorical dependent variables. LDA assumes that the conditional probability density functions $p(\vec{x} \mid y=0)$ and $\mathrm{p}(\overrightarrow{\boldsymbol{x}} \mid \mathrm{y}=1)$ are both normally distributed with different means but equal covariance. Because of this equal covariance assumption the direction in which separation is optimal is given by

$\overrightarrow{\boldsymbol{w}}=S_{W}^{-1}\left(m_{1}-m_{2}\right)$

$\overrightarrow{\boldsymbol{w}}$ is the direction of optimal separation, $\mathrm{m}_{1}$ and $\mathrm{m}_{2}$ are a vectors with the mean of each feature for class 1 and $2 . S_{w}$ is the within-class scatter matrix which is proportional to the covariance matrix of the whole dataset. Because of the equal covariance assumption, the criterion of an input $\overrightarrow{\boldsymbol{x}}$ belonging to either class is a function of the linear combination of the known features. In geometrical terms, the multidimensional feature vector $\overrightarrow{\boldsymbol{x}}$ is projected onto a line in the direction of a vector $\overrightarrow{\boldsymbol{w}}$ which produces a one dimensional signal $\mathbf{y}$. Classification of a test sample $\overrightarrow{\boldsymbol{x}}_{\text {test }}$ is done by projecting it in the direction of $\overrightarrow{\boldsymbol{w}}$, and subsequent thresholding produces the binary decision. 
Chapter 1

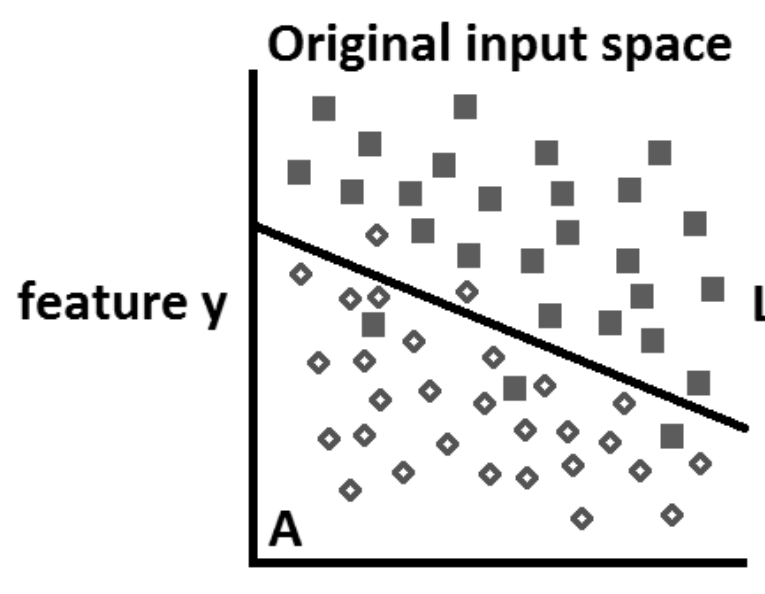

feature $x$

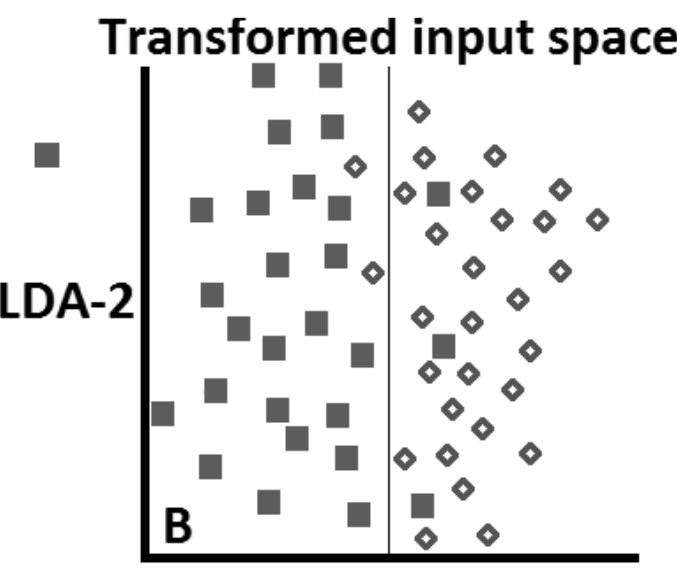

LDA-1

Figure 5 Example of an LDA classifier separating two classes (red and blue) in a 2dimensional space. The solid line indicates the decision boundary (A). The linear transformation $\overrightarrow{\boldsymbol{w}}$ found by LDA equates to a rotation such that the decision boundary becomes a vertical line (B).

Just like LDA, a Support Vector Machine (SVM) is a supervised learning and classification method that analyzes and recognizes patterns (38). Given a set of labeled training data points, SVM finds a boundary that separates the two classes while simultaneously maximizing the margin. The margin is defined as the distance from the boundary to the nearest training data point. SVM training is thus defined as an optimization problem where the margin is to be maximized. The motivation behind maximizing the margin is that in general a wider margin means that two classes can be separated with higher certainty and hence an increased generalization ability of the classifier. Furthermore the SVM only uses those training data points that are close to the decision boundary for its computations. These data points are called support vectors (SV) and explicitly define the decision boundary. In many real world problems two classes are not linearly separable in the original finite dimensional (number of features) space. A kernel function can be used to nonlinearly transform the original feature space into a much higher dimensional space in which the two classes are linearly separable (39). This is always possible in the extreme case where the number of 
Introduction

dimensions is equal to the number of training samples. Many different kernel functions can be used for this transformation, here the most widely used Gaussian radial basis (RBF) kernel is chosen:

$K\left(x_{1}, x_{2}\right)=e^{-\frac{\left\|x_{1}-x_{2}\right\|}{2 \sigma^{2}}}$

The Gaussian Radial basis function (RBF) kernel acts as a similarity measure of two data points yielding a value of 1 when the two data points are equal and a value $<1$ when they are not. The further the two points are apart in features space, the lower their similarity score. The rate at which $K\left(x_{1}, x_{2}\right)$ decreases as $x_{1}$ and $x_{2}$ move apart is determined by the kernel width parameter $\sigma$. In practice $\sigma$ determines the flexibility of the decision boundary, low values of $\sigma$ yield a highly flexible (over-fitted) boundary while for high values of $\sigma$ the equivalent of a linear SVM classifier is produced. In many real world applications the data does not only contain information relevant for the classification task but also noise and outlier values. The concept of softmargin allows for some misclassified data points if no hyperplane can be found that separates the two classes(38). The soft margin method will choose a hyperplane that separates the two classes as good as possible while still maximizing the margin between the correctly classified data points. Consequently a trade-off has to be made between the number of good classified data points and the width of the margin. This is done by giving a penalty $\xi$ for each misclassified example which is added to the objective function. The magnitude of this penalty is determined by the so called box-constraint $C$. When $C$ increases less training errors are allowed. Both $\sigma$ and $C$ are not analytically derivable and therefore need to be chosen iteratively using a grid-search. Training of the actual SVM classifier involves the convex optimization problem:

Minimize $\left(\frac{1}{2}\|w\|^{2}+C \sum_{i=1}^{L} \xi_{i}\right)$ 
Chapter 1

Subject to:

$$
y_{i}\left(w x_{i}+b\right) \geq 1-\xi_{i}, \forall_{i}
$$

where $\frac{1}{2}\|w\|^{2}$ is the width of the margin, and $C \sum_{i=1}^{L} \xi_{i}$ the penalty due to misclassified samples, and $y_{i}$ represent the class label which is -1 for the negative class and +1 for the positive class. The optimization problem is solved using Lagrangian multipliers $\alpha_{j}$. Only the training vectors that lie on or inside the margin have non-zero multipliers $\alpha_{j}$ and become SV.

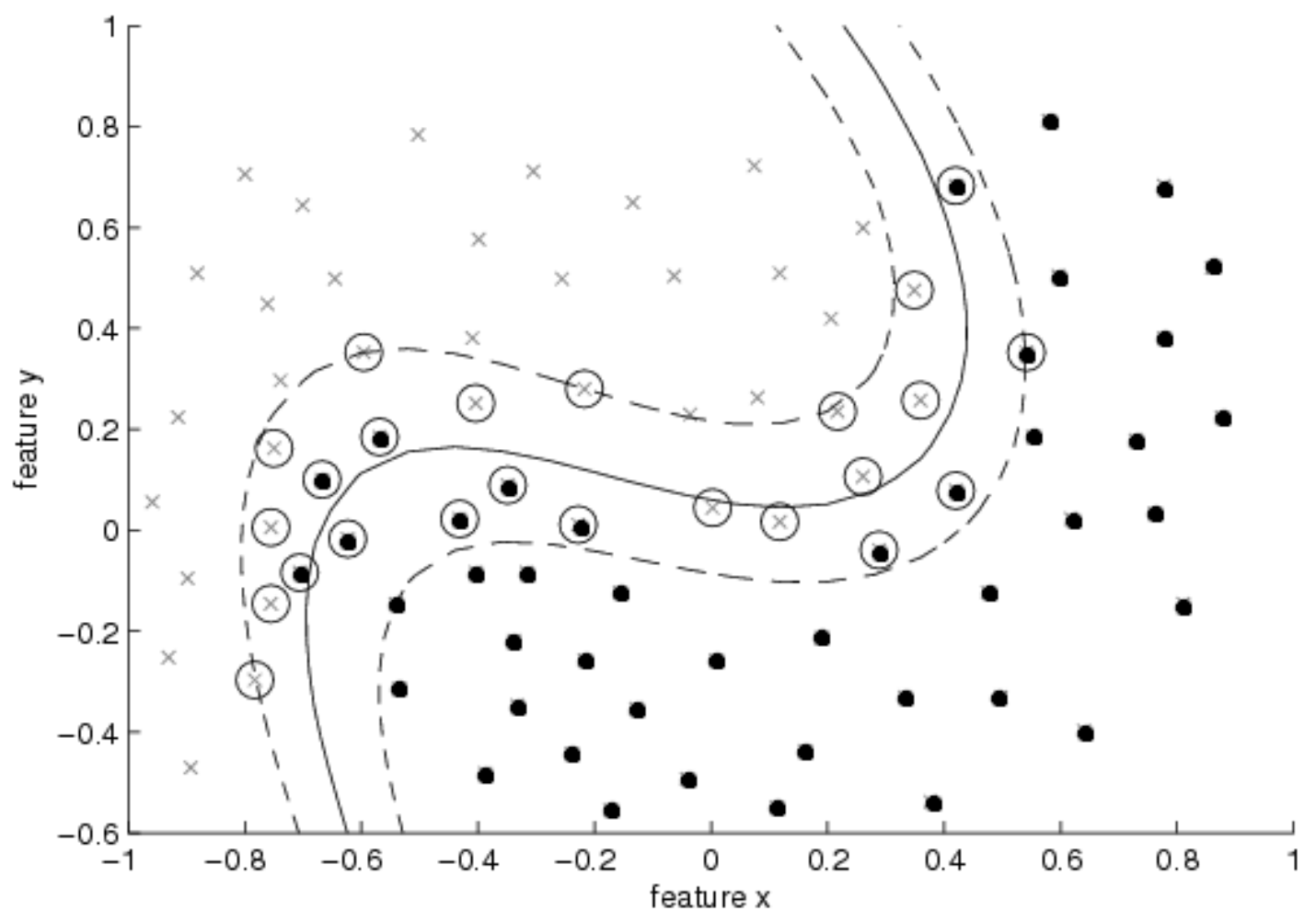

Figure 6 Example of an SVM classifier separating two classes, red and blue, in a 2dimensional space. The solid line indicates the decision boundary, defined by the Support Vectors. Each Support Vector, indicated with circles, lie on, or within the margin which is indicated by the dashed lines. 
The SVM classifier consists explicitly of SVs with each their own weigh $\mathrm{t} \alpha_{j}$. As a result, classification of new data points is performed by assigning the class-label according to the sign of:

$f\left(x_{\text {test }}\right)=\sum_{j=1}^{M} \alpha_{j} y_{i} K\left(x_{j}, x_{\text {test }}\right)+b$

$f(x)$ implies weighted sum of the kernel evaluations of the test sample with each of the M SVs. This can be seen as a kind of voting, each SV votes on its own class while the weight of this vote depends on 1: the similarity between the test sample and SV and 2: the weight $\alpha_{j}$ determined during training. In geometrical terms $f(x)$ represents the distance from a test sample to the decision boundary. The further a test point is from this boundary, the more certain the classifier is. In many problems it is convenient not only to have a classification decision but a measure of its certainty as well. It is therefore convenient to transform $f(x)$ into a probability $P(y=1 \mid x)$. This can be done by fitting a logistic regression model over the classifier's scores (40). It produces probability estimates by the following logistic transformation of the classifier output $f(x)$ :

$P(y=1 \mid x)=\frac{1}{1+e^{A f(x)+B}}$

Both $A$ and $B$ are determined by using a maximum-likelihood estimation on a portion of the training data set not used to train the SVM classifier. If $\mathrm{B} \neq 0$ the probability estimate implies a correction compared to the original decision function $\operatorname{sign}(f(x))$. In short, the two classification methods, LDA and SVM, described in the previous sections can both be used for the classification of EEG epochs. The main reason to choose either for SVM or LDA depends on the complexity of the classification problem. Therefore, in this thesis LDA will be used for the relatively simple task of asymmetry detection whereas for the more complex task of seizure detection, SVM will be used. 
Chapter 1

Performance evaluation

Classifier performance is evaluated in terms of sensitivity and specificity. Sensitivity is defined as the number of positive samples correctly classified divided by the total number of positive samples. Consequently, specificity is defined as the percentage of correctly classified negative samples. In classification tasks using SVM or LDA the classifier score is a continuous variable which after thresholding produces the classification decision. By choosing a threshold a trade-off between sensitivity and specificity can be made. Furthermore the performance over the whole range of possible thresholds can be used to compare different algorithms. Receiver operator characteristics (ROC) curves can be constructed by plotting the sensitivity-specificity pairs for every possible detection threshold (41). These curves show the potential of a classifier which can be quantified by calculating the area under the curve $(A \cup C)$. The ROC AUC represents the chance that a random positive sample will be assigned a higher classifier score then a random negative example. A value of 1 means perfect classification while 0.5 represents random classification.

\section{Aims}

Main objective of this thesis is to improve existing detection methods of epileptic seizures and asymmetric cerebral activity, which are both clinically relevant in the field of epilepsy. Nowadays intermittent visually guided analysis is currently best practice in ICU neuromonitoring, being rather time consuming and relying on the availability of a highly trained expert. Therefore, implementation of long-term ICU monitoring in daily clinical practice necessitates automated detection algorithms. A major aim of this thesis is to develop an automated seizure detection method that can be successfully used in an ICU setting. As a state of the art method in seizure detection, the SVM classifier based seizure detection method introduced by Temko et al. (42) serves as the basis for our work. We introduce several new extensions resulting in further 
optimized seizure detection performance. In case of asymmetric cerebral activity detection, we use LDA and the Brain symmetry index (BSI) introduced by van Putten et al. (43) to further optimize asymmetry detection during the Wada test.

\section{Outline of this thesis}

In the next section the content of, and relation between, the subsequent chapters is described. In the first 2 chapters more fundamental and methodological issues are addressed while in the chapters 3 and 4 a more pragmatic approach is taken to further optimize seizure detection in a realistic ICU setting. Finally, the last chapter is about asymmetry detection during the Wada test. In all chapters about seizure detection, classification is based on SVM while asymmetry detection is based on LDA.

Chapter 2 describes a new method to reduce inter-patient variability by correcting for differences in so called EEG feature baseline values. Principle is that analysis of the actual ongoing EEG is always referenced to a so called baseline EEG. In this way, we aim for creating an age independent seizure detection method. Seizure detection performance, with and without this feature baseline correction (FBC) method, is evaluated on a set of neonatal EEG recordings. This patient population was chosen because EEG differences are relatively large because of the rapid changes in neonatal EEG characteristics due to brain maturation.

In chapter 3, the FBC methodology developed in chapter 2 is used to investigate the influence of training dataset composition on age independent seizure detection performance. In particular, the major question was whether a classifier based on both neonatal and adult EEG was optimal for age independent seizure detection. To this end, classifiers based on either neonatal or adult EEG, as well as both neonatal and adult EEG, are evaluated on both patient groups. 


\section{Chapter 1}

Next, the optimal classifier based on the findings of chapter 2 and 3 was evaluated on an independent set of long term EEG registrations from comatose adult patients. The main reason for bad classification performance turned out to be caused by dynamic changes in baseline EEG characteristics. These baseline EEG characteristics were assumed to be constant when the FBC methodology in chapter 1 was developed. However this was a valid assumption for the short routine EEG registrations used in chapter 2 and 3, it was not in case of long term registrations lasting multiple days. Therefore, in chapter 4, an automated feature baseline update method is proposed that takes into account changing dynamic EEG properties which occur in long term EEG registrations. Seizure detection performance with and without this newly introduced automated baseline update algorithm is evaluated in a set of long term ICU EEG registrations of comatose patients.

So far, seizure detection was approached as a patient-independent classification task. This means that a classifier based on EEG data from a given set of patients is used to detect seizures in the EEG of an independent patient. In case of long term EEG monitoring this may be unnecessarily difficult, especially because as the monitoring goes on, more and more information about the EEG characteristics of the particular patient becomes available. Chapter 5 describes a new method to make seizure detection during EEG monitoring patient specific by using EEG segments that are classified as seizure by the algorithm.

Finally in chapter 6 another EEG classification task is investigated. Improvements in automated EEG asymmetry detection during the Wada test are presented by using both spatial and temporal EEG changes. Furthermore, the time course of asymmetry of different frequency bands during hemispheric deactivation is evaluated. 
Chapter 2

\section{EEG feature pre-processing for neonatal epileptic seizure detection}

Bogaarts JG, Gommer ED, Hilkman DMW, van Kranen-Mastenbroek VHJM, Reulen JPH. EEG Feature Pre-processing for Neonatal Epileptic Seizure Detection. Ann Biomed Eng. 2014:1-9. 
Chapter 2

\section{Abstract}

Objective: Aim of our project is to further optimize neonatal seizure detection using Support Vector Machine (SVM). Methods: First, a Kalman filter (KF) was used to filter both feature and classifier output time series in order to increase temporal precision. Second, EEG baseline feature correction (FBC) was introduced to reduce inter patient variability in feature distributions. The performance of the detection methods is evaluated on 54 multichannel routine EEG recordings from 39 both term and pre-term newborns. The area under the receiver operating characteristics curve (AUC) as well as sensitivity and specificity are used to evaluate the performance of the classification method. Results: SVM without KF and FBC achieves an AUC of 0.77 (sensitivity 0.68 , specificity 0.71 ). The highest AUC of 0.90 (sensitivity 0.80 , specificity 0.83 ) is achieved on baseline corrected features with a Kalman smoother used for training data pre-processing and a KF used to filter the classifier output. Conclusions: Both FBC and KF significantly improve neonatal epileptic seizure detection. Significance: This paper introduces significant improvements for the state of the art SVM based neonatal epileptic seizure detection. 
Feature baseline correction

\section{Introduction}

On Neonatal Intensive Care Units (NICU) many vital parameters are recorded. However monitoring brain function by electroencephalography (EEG) is rare, mainly because signal interpretation requires expert visual inspection which is very labour intensive. In 1-6\% of newborns on the NICU (sub clinical) seizures occur and even more frequent in premature and low-birth-weight children (44). Failure of seizure detection and subsequent lack of treatment can result in brain damage. Automatic EEG analysis could facilitate the application of NICU brain monitoring. Over the past years numerous methods for detection of neonatal seizures have been developed (4549). Recently, a new neonatal seizure detection method based on Support Vector Machines (SVM) was introduced (42) which outperformed existing methods. Although the results obtained with the SVM method allow for its application in neonatal intensive care units, there is still room for improvement. This in particular with respect to the correct classification of single epochs contrary to detection of seizure events(50).

An SVM classifier is trained in a supervised way to separate data into two classes, in this case seizure or non-seizure. An example of an EEG epoch without and with seizure activity is shown in figure 7 and 8 respectively. For the development and evaluation of the SVM classifier the EEG was annotated for seizure occurrence through visual inspection by an experienced electroencephalographer. Then for each (10s) EEG epoch 103 different quantitative EEG features (hereafter simply called features) are calculated per channel. In this way each epoch is represented by a feature vector for each channel which defines a point in a 103 dimensional feature space. Each epoch is labelled either seizure or non-seizure as annotated by our human EEG expert. Training of an SVM classifier implies construction of a plane in this 103 dimensional space that best separates seizure from non-seizure points. This surface is defined by a subset of the original feature vectors called the support 


\section{Chapter 2}

vectors (SV). A new unclassified EEG epoch can now be classified as seizure or non-seizure by its location with respect to this surface.

Characteristics of neonatal EEG are influenced by brain maturation (51) and may differ among EEG channels (52) so that not only properties of seizure EEG may be very different among subjects but also of "baseline" non-seizure EEG parts. Consequently the ranges of the so called baseline non-seizure features as well as their corresponding detection thresholds may vary between subjects in our data set. This may hamper optimal training of the SVM classifier. In an optimal situation the detection threshold for a feature should be the same for each patient and EEG Channel. Since this is not true, our hypothesis is that correcting features for baseline differences may improve SVM classifier performance. One major aim of this study is to explore a possible relation between detection thresholds and non-seizure feature values and to use this relation in a feature baseline correction (FBC) procedure to reduce inter patient variation. It is hypothesised that this FBC procedure results in better classification performance.

Another aim is to increase the classification performance by using a sophisticated filter algorithm. The features used for seizure detection as well as the classification output can be seen as an indirect but also "noisy" measurement of an underlying system state, in this case the presence or absence of seizure activity. A Kalman filter (KF) is an algorithm that uses a series of noisy measurements to produce estimates of the underlying system state being more precise than when based on single measurements alone. The application of Kalman filtering has recently proved to reduce the amount of false detections in a study on seizure prediction (53). A KF is especially useful for online tasks such as seizure detection as it does not introduce a computational delay in contrary to for example a central moving average filter. However the possible merits of KF in neonatal seizure detection have not yet been evaluated with regard to SVM classification. For this 
reason we also investigated KF in several stages of the SVM training and classification process. Major aims of our study are on the one hand to evaluate the effect of FBC and on the other of KF on SVM based neonatal seizure detection using routine EEG recordings. The overall goal of our work is to incorporate these findings into development of an optimal EEG seizure monitoring system to be constructed in the near future.

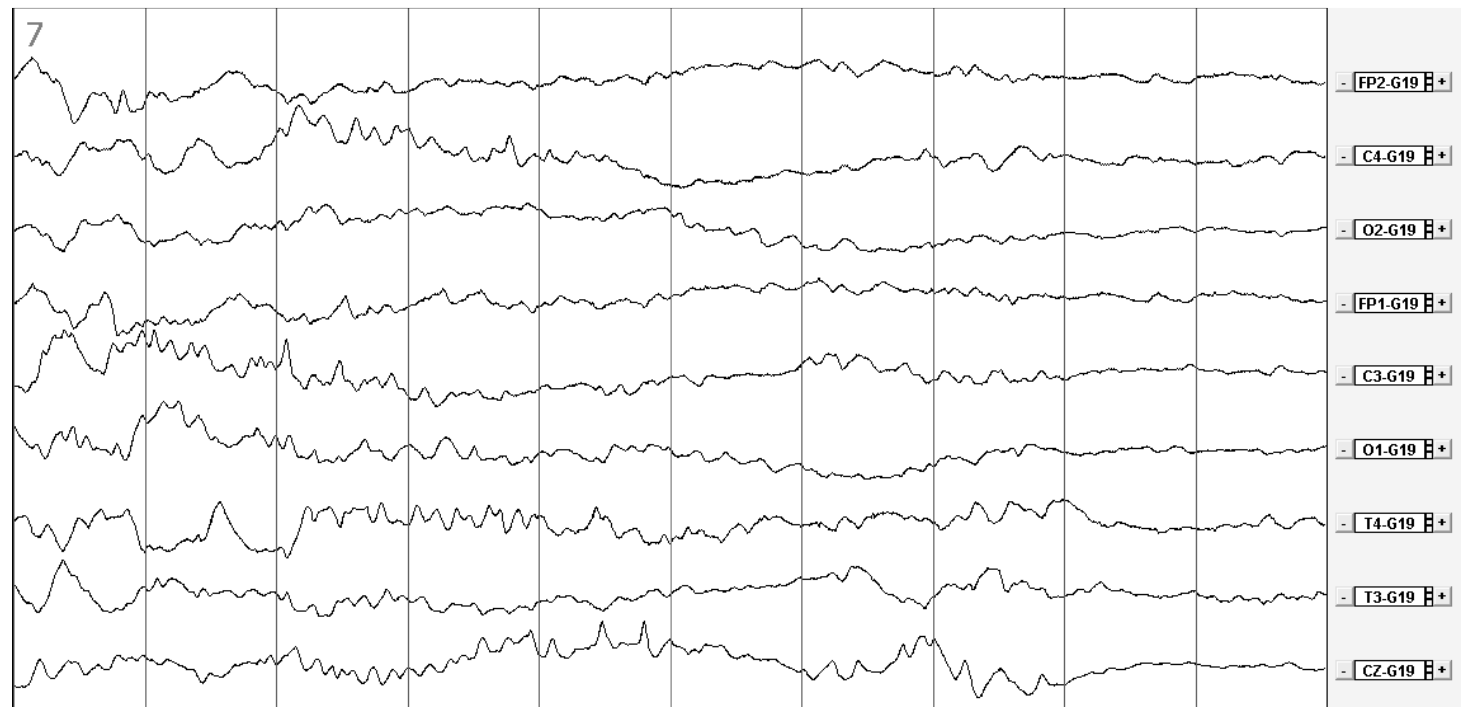

Figure 7 Example of an epoch without seizure activity.

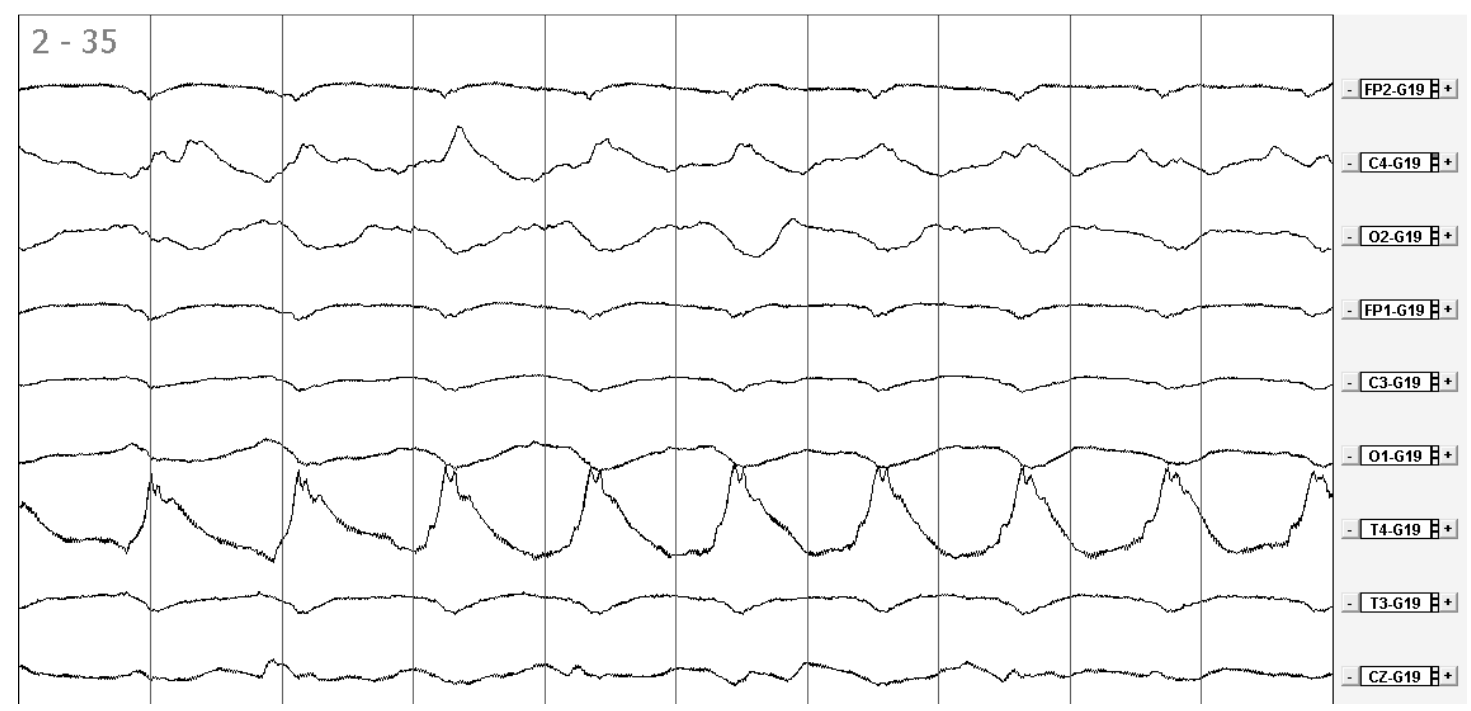

Figure 8 Example of a 10 second EEG epoch with seizure activity in all channels (generalized seizure). 
Chapter 2

\section{Materials and Methods}

\section{EEG dataset}

The dataset used in this study consist of 54 routine EEG registrations from 39 different neonates with a postnatal age between 0 and 6 months (mean gestational age: $40 \pm 5$ weeks). The EEGs were recorded between 2000 and 2012 in the Maastricht University hospital MUMC+, the Netherlands. The only inclusion criteria used are postnatal age between 0 and 6 months and the presence of seizures as indicated by a specific code in our EEG file database. Recordings were made according to the international restricted10-20 system for neonates (54) using a BrainLab EEG recording system (®OSG, BVBA, Belgium) at a sampling frequency of $250 \mathrm{~Hz}$ using a common average montage. The restricted 10-20 system of electrode placement uses nine active scalp electrodes: $\mathrm{Fp}_{1-2}, \mathrm{C}_{2}, \mathrm{C}_{3-4}, \mathrm{~T}_{3-4}$, and $\mathrm{O}_{1-2}$ electrodes. Each patient $\mathrm{EEG}$ registration contained at least one seizure. The average registration length is 26 minutes (range 10 - 95 minutes) and the dataset contains 340 annotated seizures with durations ranging from 10 seconds to 14 minutes. The minimal length of a seizure to be annotated was 10 seconds following the recommendations of the International Federation of Clinical Neurophysiology (54). Per channel annotations are provided by an experienced EEG technician.

\section{EEG pre-processing and feature extraction}

EEG recordings are band pass filtered between 0.5 and $32 \mathrm{~Hz}$ and are partitioned into 10 s epochs with 5 seconds (50 \%) overlap between epochs. The complete dataset consists of 18658 epochs of which 5402 are seizure, 11950 are non-seizure and 1306 epochs contain both seizure and non-seizure EEG. For each epoch 103 features are 
calculated that are generally used for seizure detection (34-36). These features stem from different signal description domains such as time, frequency and information theory. Each EEG epoch is then characterized by a 103 dimensional feature vector (FV) which is used for SVM classifier training and subsequent classification. Epochs containing both seizure and non-seizure EEG, i.e. those epochs at the start and end of a seizure are not used for training and testing.

\section{Support Vector Machine principles}

A SVM is a discriminative model which uses a subset of the training data to construct a surface that separates seizure from non-seizure feature vectors $(39,55)$. Using a radial basis function ( $r b f)$ kernel, the data is transformed from the original 103 dimensional feature space into a higher dimensional space. In this way a complex non-linear classification problem may be converted into a simpler linear problem. Only the feature vectors that are close to the decision surface are used by the SVM. As illustrated in figure 10, classification consists of training and testing. To obtain an unbiased indication of future performance of a classifier it is essential that the data used for testing has not been used in any way during training. Therefore training and testing is performed using a so called "leave one out" (LOO) cross validation scheme. This means that a classifier is trained on all but one patient's data and this classifier is then used to classify the epochs of the left out patient. This is repeated for each of the 39 patients in the dataset.

To create the training set, from each registration a maximum of 2 minutes (24 epochs) of seizure EEG is selected for each channel and usually contains epochs from several seizures. This is done to make sure that all measurements are represented equally in the training set. Seizure epochs are selected per measurement based on the average distance to the non-seizure epochs using the kernel function also used for SVM training. In this way those seizure epochs that are most clearly seizure are selected. For the non-seizure class 1000 epochs per channel 


\section{Chapter 2}

are chosen at random from the complete dataset also taking into account the difference in number of non-seizure epochs per measurement. The features in the training data are normalized by subtracting the mean and dividing by the standard deviation to make the various features commensurable. Normalization of the test data is performed with the means and standard deviations of the training data. During training a separating surface in the feature space is constructed that maximizes the margin between seizure and non-seizure epochs. A kernel parameter $(\sigma)$ and a generalization parameter $(C)$ are determined which maximizes the classification performance in five-fold cross validation on the training data. A SVM classifier is then trained with these two parameters on all training data. Subsequently, the performance of this model is evaluated on all data of the left out patient. Final performance metrics, as explained in the last paragraph of the methods section, are calculated for the complete dataset as if only one classifier was used to classify the data of all 39 patients.

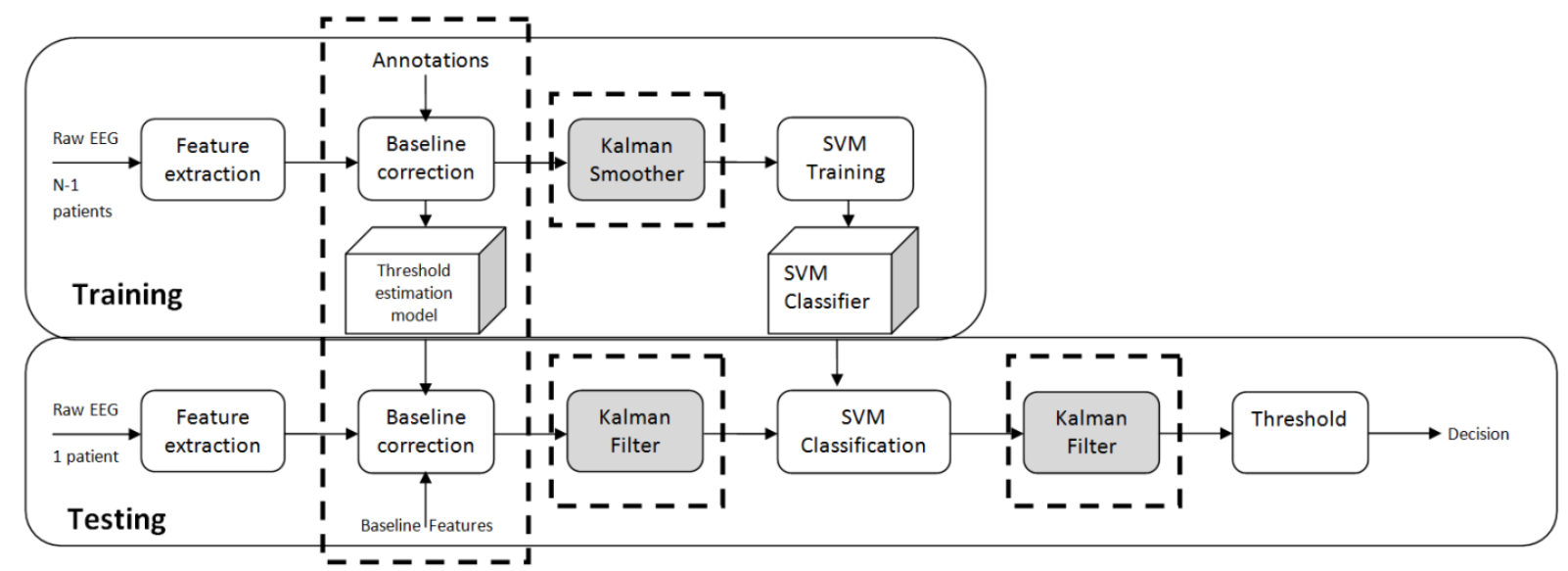

Figure 9 Various stages of SVM training and testing. Training is done on all but one $(\mathrm{N}-1)$ patient's data. Testing data are from the left out patient. Training and testing scheme is repeated so that every patient has been a test subject. KF can be applied at three stages (shaded boxes). In case of FBC it is always applied in both the train and test phase. 


\section{Feature Baseline correction procedure FBC}

In figure 10, a problem is illustrated when the values of a specific feature of two patients cover a different range. In this example of feature 'Log Activity' a threshold of around 2 will optimally separate the seizure (S) from the non-seizure (NS) epochs of patient 5. However, application of this criterion would be far from optimal for patient 10 . To solve this, we would like to estimate optimal settings based on baseline NS EEG features.

The FBC procedure for a single feature can be described as follows: for each recording per channel containing seizure activity, the average NS feature value (ANSFV) and an optimal threshold (Topt) is determined that best separates the $S$ from NS epochs. So in case of a single feature, $N$ recordings with $M$ involved channels result in $N^{*} M$ Topt values which may scatter over a broad range. From these epochs the average of the non-seizure feature values (ANSFV) are computed. This results for $\mathrm{N}$ recordings into N ANSFV values. Figure 11 shows for 54 recordings a scatter plot of Topt versus ANSFV for the feature "log total power" illustrating a linear regression line. Each point represents Topt-ANSFV pair from a registration in the training set. In case of a new untested EEG recording from a 3 minute segment of EEG at the start of the recording, epochs without artefacts, epileptic or epileptiform activity are selected to serve as a baseline. From these segments the ANSFV are calculated. This ANSFV is then used to estimate Topt using the linear relation between Topt and ANSFV derived from the registrations in the training data. In this way variation in Topt due to different baseline EEG properties is corrected for. Outliers as well as Topt-ANSFV pairs from a channel with an AUC value below 0.7 are excluded before the linear relation is determined. 
Chapter 2

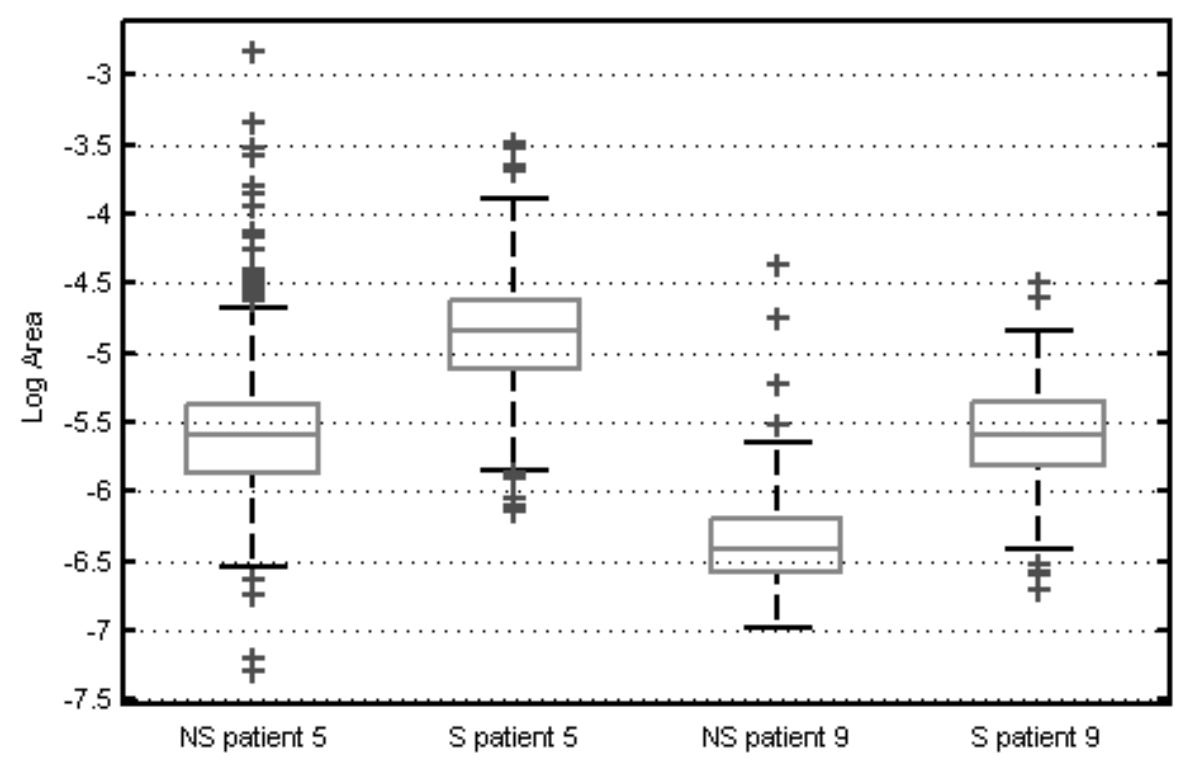

Figure 10 Seizure (S) and non-seizure (NS) distribution of feature "Log activity" for patient 5 and patient 10 (Channel $C_{Z}$ ). The crosses represent outlier feature values. IT can readily be seen that the distributions from the two patients cover different ranges.

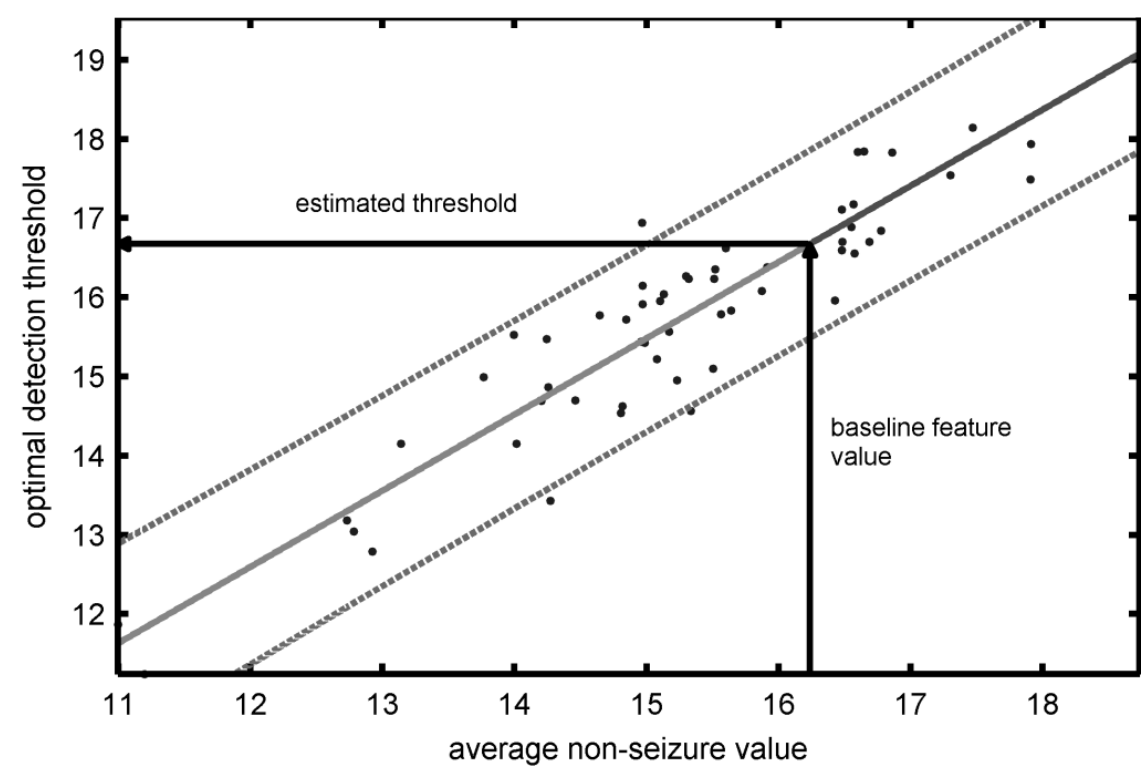

Figure 11 Optimal detection threshold, Topt, for feature Log total power plotted versus its average non-seizure baseline feature values for 56 training EEG recordings. The regression line is used to estimate Topt (16.8) for the testing data as indicated by the arrows in case of an ANSFV of 16.2. 
The described FBC method is performed for every feature, resulting in 103 feature specific scatter plots of Topt vs. ANSFV. In case there is no significant correlation between Topt an ANSFV the slope and offset of the linear relation are both set to 0 which effectively means that $F B C$ is not performed. In this way in case of a new EEG registration, based on a baseline EEG segment, 103 Topt values for each of the features are calculated and are thereupon used in the classification process. The same method is also applied to obtain estimates for Topt for channels in the training dataset that do not contain seizures.

\section{Kalman Filter}

Recently a KF in combination with SVM was evaluated in seizure prediction (53) resulting in significantly decreased false positive rate in seizure prediction. Several variations of the KF exist which differ in principle in the kind of measurements used for the estimation of the system state at a certain moment in time. The normal KF uses only past measurements which makes it an online process without time delay. In case of offline filtering a fixed interval Kalman smoother (KS) uses all available measurements (56). As illustrated in figure 9, for the training phase fixed-interval Kalman smoothing can be applied to the feature

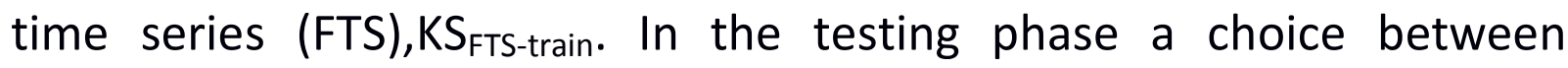
Kalman filtering of the feature time series $\left(\mathrm{KF}_{\mathrm{FTS} \text {-test }}\right)$ and the SVM classifier output $\left(\mathrm{KF}_{\mathrm{SVM}}\right)$ can be made. Preliminary results showed that $\mathrm{KF}_{\mathrm{SVM}}$ and $\mathrm{KF}_{\mathrm{FTS} \text {-test }}$ yielded no significant differences in performance. However application of $\mathrm{KF}_{\mathrm{FTS} \text {-test }}$ involves 103 filter operations per EEG epoch while $\mathrm{KF}_{\mathrm{SVM}}$ only involves one filter operation per epoch which is preferable considering the computational time restrictions during testing. KS is also applied to the SVM classifier output to illustrate how a trade-off can be made between (statistical) reliability of the filter estimate and delay introduced by the filter. A detailed description of the principles of KF can be found in appendix $A$. 
Chapter 2

SVM training and classification procedure

Figure 9 shows the different stages of the training and classification procedure. The feature vectors extracted from the training EEGs are pre-processed before they are used to construct a SVM classifier. Pre-

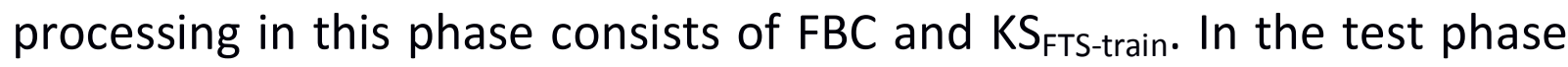
$\mathrm{KF}_{\text {SVM }}$ is performed as a post-processing step. By including or omitting one or more of the operations schematized by the dashed boxes in figure 9, different train-test scenarios can be set up and evaluated. Table 1 shows the 6 scenarios used in this study. All calculations are performed in MATLAB (57). The SVM classifier is used to classify each EEG channel separately. The classifier output from all channels are combined using the OR operator. This means that only detection in one channel is needed to trigger the detection alarm. This is equivalent to applying the MAX operator to the 9 classifier outputs.

\begin{tabular}{cccc}
$\begin{array}{c}\text { Train- } \\
\text { test } \\
\text { scenario }\end{array}$ & $\begin{array}{c}\text { Filtering of } \\
\text { classifier } \\
\text { output } \\
1\end{array}$ & $\begin{array}{c}\text { None } \\
\text { Filtering of } \\
\text { train-FTS }\end{array}$ & FBC \\
2 & KF & None & No \\
3 & None & None & No \\
4 & KF & None & Yes \\
5 & KF & KS & Yes \\
6 & KS & KS & Yes \\
\hline
\end{tabular}

Table 1 The 6 different train and test scenarios evaluated in this paper. In the first 4 scenarios no filtering is applied to the FTS during training. In scenario 5 and 6 the FTS in the training phase are filtered using a KS. FBC is applied in scenario 3-6. 
Feature baseline correction

Metrics and statistical tests

Considering the fact that our dataset consists of relative short EEG registrations with often a very low amount of seizure epochs, performance metrics are calculated for the dataset as a whole. A reliable seizure detection algorithm should accurately classify both seizure and non-seizure epochs. A Receiver Operating Characteristics (ROC) curve shows how the sensitivity of a classifier varies as a function of its specificity. The area under the ROC curve (AUC) reflects the performance over all possible operating points and has a value of 1 for perfect classification and 0.5 for random classification. In this study, AUC of the SVM classifier output is used to compare the different classification configurations in this study and to compare its outcome with existing methods described in the literature. To compare the AUC of two different methods tested on the same data the approach of Hanley et. al (58) is used to calculate the z-statistic of the AUC values using the standard errors of the AUC values. P-values less than 0.05 are considered significant. Additional to the AUC values also the sensitivity and specificity at the operating point where there sum is maximal are provided. These metrics might give a more intuitive view of classification performance especially in a clinical setting.

\section{Results}

\section{SVM Classification results}

Figure 12 shows the ROC curves of the different train-test scenarios and the corresponding AUC values are depicted in figure 14. All reported differences in AUC values differ statistically significantly from each other $(p<0.01)$. The original classification system (scenario 1 ) achieves an AUC of 0.77 (sensitivity 0.68 , specificity 0.71 ). Application of KF to the SVM output (scenario 2) increases AUC to 0.80 (sensitivity 0.79 , specificity 0.75). FBC (scenario 3 ) resulted in an increase of AUC to 0.86 (sensitivity 


\section{Chapter 2}

0.68 , specificity 0.71 ). Using both $\mathrm{FBC}$ and $\mathrm{KF}_{\mathrm{SVM}}$ (scenario 4) further increases the AUC to 0.89 (sensitivity 0.76 , specificity 0.84 ).

\section{Training data smoothing}

As can be seen in figure 12 and 13, application of the KS to the FTS in the training phase (scenario 5) further increases the AUC to 0.90 (sensitivity 0.80 , specificity 0.83 ). Also the number of SV of the classifiers trained on filtered FTS is significantly lower compared to the unfiltered versions. The number of training vectors retained as SV is reduced from $57 \%$ to $41 \%$ without FBC and from $43 \%$ to $29 \%$ with FBC.

\section{Offline detection}

An upper bound for the AUC of 0.91 (sensitivity 0.80 , specificity 0.86 ) on achievable performance by filtering in the test phase is obtained by applying a KS instead of a KF to the SVM classifier output (scenario 6).

\section{Relation between ANSFV and Topt.}

To substantiate FBC the relations between average non-seizure feature values, optimal detection thresholds and 3 minute baseline feature values are evaluated. FBC is based on the observation that a relation exists between ANSFV and Topt as described in the methods section. The strength of this relation is given by the linear correlation coefficient which proved to be significant $(p<0.05)$ for all but two features (peak frequency and $15^{\text {th }}$ cepstral coefficient). Exclusion of these 2 features from the feature-set did not significantly change classification performance (results not shown). 
Feature baseline correction

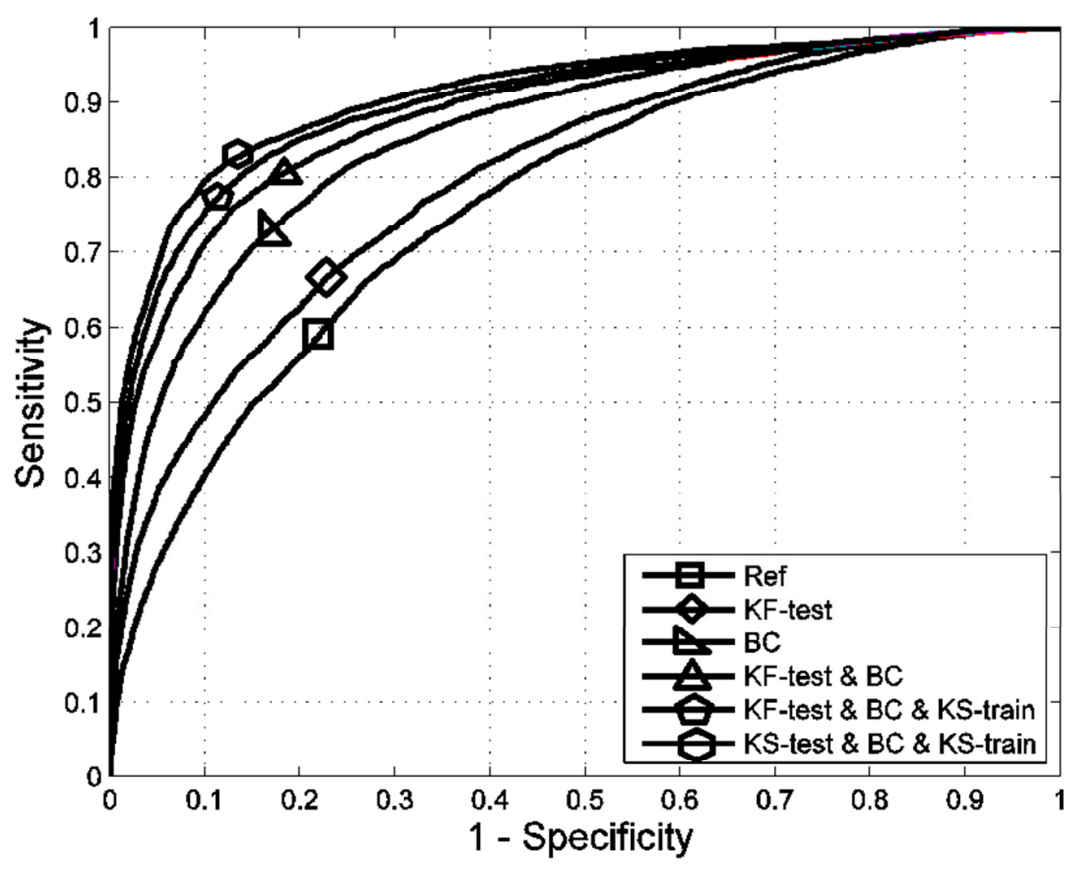

Figure 12 ROC curves of the various train-test scenarios. The ROC curve of each consecutive scenario shifts towards the upper left corner, hence achieving better performance.

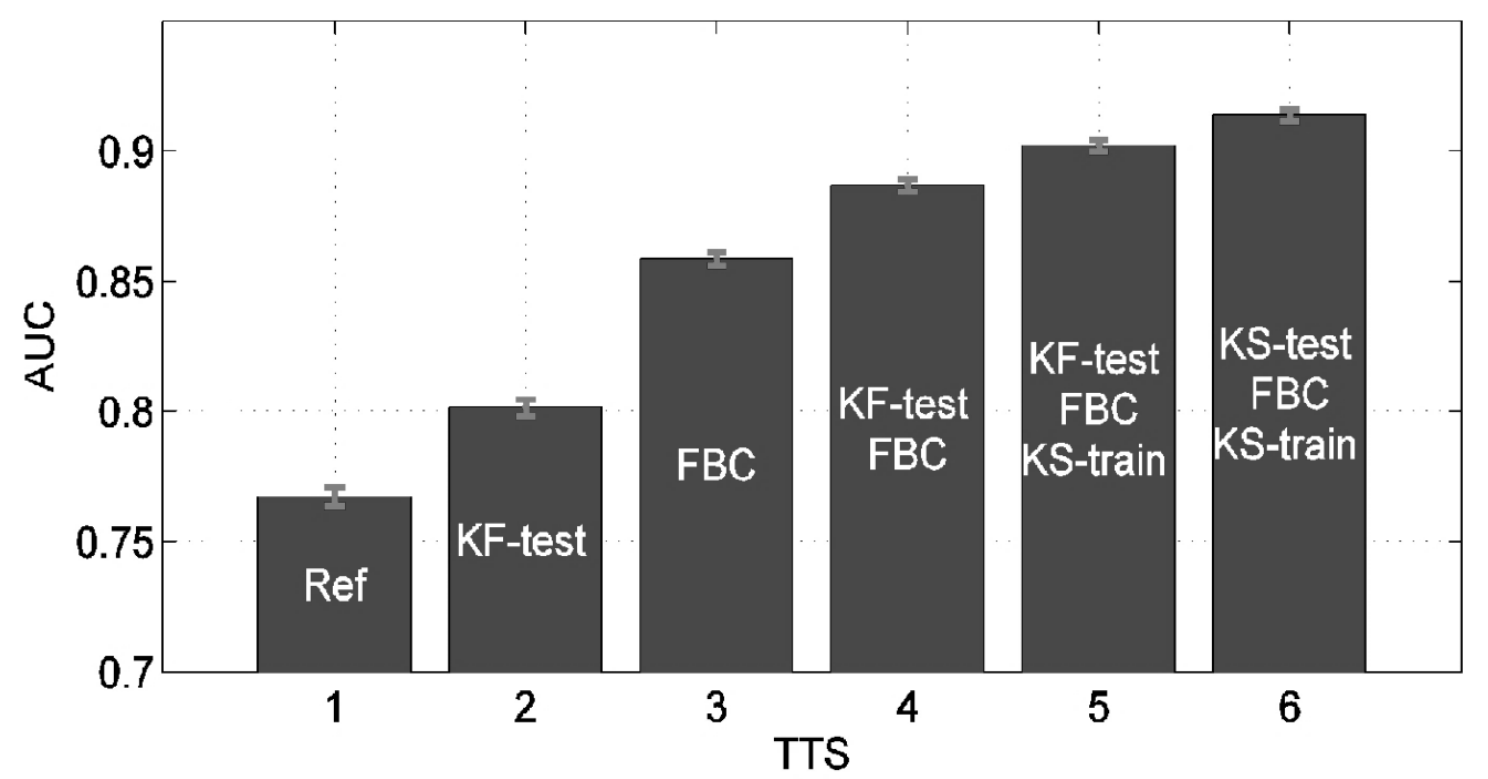

Figure $13 \mathrm{AUC}$ values for the various train-test scenarios. The best performance is achieved with FBC, filtering of the classifier output, and smoothing of the FTS in the training phase. 
Chapter 2

\section{Discussion}

The final aim of our work is the development of an optimal intensive care cerebral monitoring system. This study addresses the optimization of EEG epoch classification by introducing FBC and KF. To evaluate the effect of $F B C$ and KF on seizure detection performance we used only epoch based metrics because we believe this best reflects the actual performance of a classification method. Event related metrics to express performance could be useful in the last stage of development were the best way of clinical implementation is sought. Correction of other physiological parameters like ECG, EMG, respiration as well as an EEG artefact detection algorithm should also be part of such a system but are beyond the scope of this study. It must be emphasized that inclusion of the aforementioned aspects certainly will improve SVM performance.

Results can be evaluated per patient, averaged over patients, or for the complete dataset at once. Because of the very low amount of seizure epochs in some registrations, we chose for the second option. However, averaging per patient results yielded the same outcome (results not shown) and therefore in our opinion supports the conclusions.

\section{Feature baseline correction}

In this work a FBC method is developed motivated by the observation that typical seizure and non-seizure EEG feature values may cover different ranges for individual patients. To eliminate these inter patient differences an optimal detection threshold per patient per feature is determined. These thresholds are then subtracted from the feature values. For each patient the features values are now theoretically distributed around 0 . Unlike for the training dataset, optimal detection thresholds for a test measurement cannot be determined in advance. 
They can be estimated by first computing an average non-seizure feature value of a manually selected non-seizure baseline part of the EEG. Then, based on the linear relation between Topt and the ANSFV, its optimal threshold Topt is extrapolated. This procedure requires initially a selection of a non-seizure reference EEG segment, in this study of a length of 3 minutes. We did not study whether selection of shorter or longer reference segments would improve the result. As a result the classification performance SVM based detection is significantly improved. Moreover a reduction of SVM classifier training time by about $\sim 40 \%$ and a reduction of the classifier complexity as reflected in the number of SVs are both in favour of the FBC method.

\section{Kalman filtering}

KS was applied to the FTS in the training phase and KF to the classifier output (KF) in the test phase (figure 9). Smoothing in the training phase resulted in SVM classifiers with a smaller number of SVs compared to classifiers based on unfiltered features. In the test phase applying a KF to either the feature time series or the classifier output time series improved the classifier equally well. Although KF improved average performance it should be approached with some caution. With an epoch length of 10 seconds and 50\% overlap between epochs only epileptic seizures with a length of at least 15 seconds are guaranteed to fully overlap with at least one epoch. Seizures of shorter duration may risk of not being fully covered by an epoch. In case a seizure is only (partly) captured by one epoch the KF would consider this an abnormality and thus filter it out. A logical solution would be to decrease the length of the epochs or to increase the overlap between consecutive epochs. However, decreasing the length of the epoch will affect features derived from the spectral domain and probably other features as well. For these reasons, the effect of decreasing epoch length with different overlap will be part of our future research. 
Chapter 2

\section{Limitations of this study}

Furthermore the length of the EEG recordings used does not reflect a situation in which patients may be monitored for up to several days. Our dataset does not contain recordings with e.g. complete wake-sleep cycles which are typically 1 hour in duration (59). Each stage of this cycle is characterized by specific EEG patterns which could affect baseline feature values. It should therefore be investigated further whether a fixed baseline is suitable for long term monitoring or that it should be updated continually or periodically in order to adapt to changes in the baseline EEG. Also the heuristically chosen 3 minute length of the baseline segment could affect the performance of FBC especially when the baseline gets updated. Currently no distinction is made between burst and suppression patterns that might occur in the baseline. In case of a burst suppression pattern it might be better to divide the EEG into burst and suppression segments and use only either of them for the ANSFV estimation. This could also apply to the classification stage were segments of suppression or burst activity only could be classified separately.

The dataset in this study contains no EEG recordings without seizures; it is therefore not clear how the baseline correction performs on this category of recordings. In future work the performance of the FBC method will be evaluated on a large group of patients without epileptic seizures but possibly other abnormalities like burst suppression, trace alternant etc. Since there is usually an abundance of registrations without epileptic seizures these could also be incorporated in the training process in order to increase the specificity of the SVM classifier.

\section{Conclusion \& Future work}

Our work focused on developing an algorithm for correct classification of individual EEG epochs. It illustrates the usefulness of a KF and 
introduces a feature baseline correction method using standard EEG registrations. The absolute performance values reported here serve to demonstrate the benefit of $\mathrm{FBC}$ and $\mathrm{KF}$ and should not be interpreted as the final performance of a complete detection system on long-term EEG registrations. Incorporation of an artefact detection algorithm and other measurement modalities like electrooculography, electromyography, electrocardiography and respiration will certainly contribute to improved performance and for that reason will be explored in further research. In our current approach a distinction is made between EEG epochs containing either epileptic activity or none. EEG signals free from epileptic activity may include other activity types like burst suppression and/or different sleep stages. Future work will also focus on the classification of other EEG patterns and phenomena in order to develop a complete brain function monitoring platform. In our work we used all available epochs for training which is likely to be sub optimal. Ways to automatically select optimal training datasets will be subject of future research. Further research will also be devoted to identify which seizures are difficult to detect and whether it is better to distinguish different seizure types like for example spike-trains or spike-wave complexes and develop specific classifiers that target these different seizures.

\section{Appendix}

It is assumed that epilepsy is observed through the continuous and noisy features $z_{k}$ calculated from the EEG. By modelling the behaviour of $z_{k}$ by means of a state space model the KF can be applied to obtain a filtered version $\hat{f}_{k}$ of the noiseless feature value $f_{k}$. The behaviour of $z_{k}$ can be modelled as the result of a noisy measurement process:

$z_{k}=f_{k}+v_{k}$ with $v_{k}$ the zero-mean measurement noise with standard deviation $\sigma_{v}$. Furthermore it is assumed that $f_{k}$ undergoes smooth transitions between seizure and non-seizure states. This behaviour can be enforced by a white noise acceleration model (WNA) 


\section{Chapter 2}

with a nearly-constant rate of change(60). We now introduce the state vector $s_{k}=\left[f_{k}, \dot{f}_{k}\right]^{\prime}$ with $\dot{f}_{k}$ the rate of change of $f_{k}$. The signal $z_{k}$ can now be described by the following state-space model:

$$
\begin{gathered}
s_{k+1}=\left[\begin{array}{ll}
1 & T \\
0 & 1
\end{array}\right] s_{k}+w_{k} \\
z_{k}=\left[\begin{array}{ll}
1 & 0
\end{array}\right] s_{k}+v_{k}
\end{gathered}
$$

Equation 7

$\mathrm{T}$ represents the sample interval and $w_{k}$ the zero-mean process disturbance with covariance

$$
Q=\left[\begin{array}{cc}
\sigma_{w}^{2} \frac{T^{3}}{3} & \sigma_{w}^{2} \frac{T^{2}}{2} \\
\sigma_{w}^{2} \frac{T^{2}}{2} & \sigma_{w}^{2} T
\end{array}\right]
$$

Equation 8

$\sigma_{w}$ is the assumed standard deviation of the random fluctuations of $\dot{f}_{k}$. The KF can now be applied to equation 7 in order to recursively calculate a filtered estimate $\hat{f}_{k}$ of $f_{k}$. Instead of the calculated feature $z_{k}$ the filtered estimate of the noiseless feature $\hat{f}_{k}$ will be used in the training and classification procedure. The gain of the KF only depends on the ratio $\sigma_{K F} \triangleq \sigma_{w} / \sigma_{v}, \sigma_{v}$ is set to 1 and $\sigma_{w}$ is varied from $10^{-6}$ to $10^{-2}$ (in 9 equally log-spaced steps) in order to tune the filter's performance. Different optimal filter gains $\left(\sigma_{w}\right)$ were found for the KFtest $\left(1 * 10^{-3}\right)$ and the KS-train $\left(1 * 10^{-3}\right)$ in the train phase.

The amount of filtering that is applied by the KF depends on the gain $\sigma_{w}$ which is the only parameter that has to be set by the user. The value of $\sigma_{w}$ will be varied to tune filter performance. 
Chapter 3

Optimal training data set composition for SVM based, age-independent, automated epileptic seizure detection

Bogaarts JG, Gommer ED, Hilkman DM, van Kranen-Mastenbroek VH, Reulen JP. Optimal training dataset composition for SVM-based, ageindependent, automated epileptic seizure detection. Medical \& biological engineering \& computing. 2016;54(8):1285-93. 
Chapter 3

\section{Abstract}

Automated seizure detection is a valuable asset to health professionals, which makes adequate treatment possible in order to minimize brain damage. Most research focuses on two separate aspects of automated seizure detection: EEG feature computation and classification methods. Little research has been published regarding optimal training dataset composition for patient-independent seizure detection. This paper evaluates the performance of classifiers trained on different data sets in order to determine the optimal data set for use in classifier training for automated, age-independent, seizure detection. Three datasets are used to train a support vector machine (SVM) classifier: 1: EEG from neonatal patients, 2: EEG from adult patients and 3: EEG from both neonates and adults. To correct for baseline EEG feature differences among patients feature normalisation is essential. Usually dedicated detection systems are developed for either neonatal or adult patients. Normalisation might allow for the development of a single seizure detection system for patients irrespective of their age. Two classifier versions are trained on all three datasets: one with feature normalization and one without. This gives us six different classifiers to evaluate using both the neonatal and adults test set. As a performance measure the area under the receiver operating characteristics curve (AUC) is used. Evaluation of seizure detection performance for these 6 classifiers demonstrates that optimal performance is achieved with feature normalisation and a classifier trained on neonatal EEG. Final seizure detection performance in terms of AUC values is 0.914 for the neonatal, and 0.936 for the adults test set. A classifier trained on both neonatal and adult EEG yields no further performance gain. Our results underline the under-appreciated importance of training dataset composition with respect to accurate age-independent seizure detection. 
Optimal training dataset composition

\section{Introduction}

In Intensive Care Units (ICU) many vital parameters are recorded. However monitoring brain function by electroencephalography (EEG) is rare, mainly because signal interpretation requires expert visual inspection which is very labour intensive. One to six percent of newborns in the neonatal ICU experience (sub clinical) seizures. The figures for premature and low-birth-weight children are even higher [1]. It is estimated that the incidence of non-convulsive seizures (NCS) in adult patients with coma can be up to $48 \%$ which is much higher than suggested by clinical suspicion alone [2-6]. Automated seizure detection is a valuable asset to health professionals, which makes early treatment possible in order to minimize brain damage. In past years, numerous neonatal seizure detection methods have been developed [7-12]. Although reported seizure detection performance is often good enough for clinical application, there is still room for improvement. Furthermore performance is often much worse when a classification method is actually applied in a real clinical setting [13]. Automated EEG based seizure detection research has mainly focused on two separate aspects: EEG feature computation and classification methods [14]. As far as the authors are aware, little research has been published regarding optimal training dataset composition for epileptic seizure detection. As seizure detection performance depends on what a classifier has learned during training, the importance of an optimal training dataset is evident. To compose an optimal training dataset for epileptic seizure detection two distinct EEG datasets were used: neonatal and adult EEG registrations. Neonatal seizure detection is considered much more difficult compared to adult seizure detection. This is because adult seizures are generally characterized by less complex waveforms, mainly due to the completed maturation of the adult brain [15]. Until now most neonatal seizure detection procedures are based on adapted adult seizure detection systems [7]. Regarding the marked EEG differences between neonates and adults, the development of a specific neonatal classifier is 


\section{Chapter 3}

warranted [16]. It has been shown that a Support Vector Machine (SVM) classifier trained on neonatal EEG data can successfully be used for the detection of epileptic seizures in adults [15]. Moreover, our recently introduced feature baseline correction (FBC) technique compensates for differences in feature values between neonatal patients [17]. This FBC technique might also reduce differences in EEG features values between adults and neonates. If so, neonatal and adult EEG datasets can be combined to train an SVM classifier for accurate seizure detection in both adults and neonates. The objective of this paper is to evaluate the neonatal and adult seizure detection performance of three different SVM classifiers trained on: one trained on neonatal EEG, one trained on adult EEG and one trained on both neonatal and adult EEG. This evaluation is done with and without FBC to investigate the relevance of $\mathrm{FBC}$ in age independent seizure detection. Our overall goal is to gain insight into optimal training set composition for age independent seizure detection. We hypothesize that FBC enables optimal age-independent seizure detection using an SVM classifier trained on the combination of neonatal and adult EEG. In addition to comparing classification performance, specific properties of the classifiers themselves will be investigated to gain more insight into the compatibility of neonatal and adult data sets. An SVM classifier consists of support vectors (SVs), i.e. those training vectors considered most relevant for classification. By analysing the SV composition of the classifiers trained on both adult and neonatal EEG, further insight can be gained into the relative importance of each patient subset for classifier training. Because of the more complex seizure waveforms seen in neonates, the neonatal dataset will likely contribute more SVs to the SVM classifier than the adult dataset. 
Optimal training dataset composition

\section{Materials and methods}

\section{Dataset}

The dataset used in this study consists of 2 different subsets. The first dataset titled Neo consists of 54 routine EEG registrations from 39 different neonates with a mean post-conceptional age of 39 weeks (range: 28-59 weeks). The second dataset titled Adults consist of 41 routine registrations from 39 adult patients (mean age: 53 years, range: 22-84 years). The only inclusion criterion used for both datasets was the presence of at least one epileptic seizure per recording. The EEGs were recorded between 2000 and 2014 in the Maastricht University hospital, MUMC+, in the Netherlands. Further information about the patients used in this study is available in the supplementary information. The recordings for the Neo dataset were made according to the international 10-20 electrode configuration system for neonates ( 9 electrodes) [18]. For the Adults dataset the recordings were made using the full 10-20 electrode set (19 electrodes).

\section{Feature extraction}

EEG recordings were recorded at a sample frequency of $250 \mathrm{~Hz}$, band pass filtered between 0.5 and $32 \mathrm{~Hz}$ and subsequently down sampled to $25 \mathrm{~Hz}$. The EEG is then partitioned into 10s epochs with 5 seconds (50\%) overlap between epochs. Table 2 lists the 103 quantitative EEG features computed per epoch for each uni-polar EEG channel as described in the literature for neonatal seizure detection [19, 20]. These features are not only used for neonatal seizure detection but in many different subject groups [21]. They stem from different signal description domains such as time, frequency and information theory. Each EEG epoch is now described by a feature vector per channel. The Neo dataset is composed of 21855 seizure and 141277 non seizure 


\section{Chapter 3}

feature vectors (FVs). The Adults dataset is composed of 13043 seizure and 265470 non seizure FVs.

\begin{tabular}{c}
\hline EEG features \\
Total power (0-12Hz) \\
Peak frequency of spectrum \\
Spectral edge frequency (SEF80\%, SEF90\%, SEF95\%) \\
Power in 2Hz wide sub-bands (0-2 Hz, 1-3 Hz, ...10-12 \\
Hz) \\
Normalized power in same sub-bands \\
Wavelet energy (Db4 wavelet coefficient \\
corresponding to 1-2 Hz) \\
Curve length \\
Number of maxima and minima \\
Root mean square amplitude \\
Hjorth parameters (activity, mobility and complexity) \\
Zero Crossing Rate (ZCR), ZCR of the $\Delta$ and the $\Delta \Delta$ \\
Variance of $\Delta$ and $\Delta \Delta$ \\
Autoregressive modeling error (AR model order 1-9) \\
Skewness \& Kurtosis \\
Nonlinear energy \\
Shannon entropy, - Spectral entropy, \\
Singular Value Decomposition entropy \\
Fisher information \\
Linear filterbank: 15 sub-band energies (0-2 Hz, 1-3 Hz, \\
...14-16 Hz) \\
15 cepstral coefficients \\
15 second order frequency filtered bank energies \\
\hline Peak-peak voltage
\end{tabular}

Table 2 EEG features extracted for each epoch

Feature baseline correction (FBC)

To correct for differences in EEG features, normalization is essential in patient-independent algorithms [17,22]. EEG characteristics change with brain maturation and may differ among EEG channels [23,24]. 
Furthermore, not only seizure EEG properties may differ among subjects but also their "baseline" non-seizure EEG and may therefore hamper optimal SVM classifier training. Optimally, every single feature detection threshold should be the same for each patient and EEG channel. Since this is not true, FBC attempts to achieve this equality by estimating each feature's optimal detection threshold. FBC can be seen as a form of normalization and is performed for every feature per EEG channel for each patient separately. It involves the calculation (in training phase) or estimation (in test phase) of a feature specific (optimal) detection threshold which is then subtracted from the calculated feature value. In the training phase in case of a channel containing seizure epochs the feature specific threshold is calculated using the seizure annotations. For nearly every feature, a linear relation exists between the optimal threshold (Topt) and the average nonseizure feature values (ANSFV). This relation is quantified from the training dataset by fitting a linear regression model through the ANSFVTopt points. Using this relation Topt can be estimated when some nonseizure epochs are available to calculate ANSFV.. A small number of visually selected (seizure and artefact free) EEG epochs from the first 3 minutes of the registration are used to calculate the ANSFV values. The number of epochs used for this baseline depends on the availability of suitable artefact and epileptiform free EEG but are usually but not necessarily taken from the first 3 minutes of the registration.

\section{Training datasets and test procedure}

This study evaluates 3 different training data sets: 1: neonatal EEG (Neo), 2: adult EEG (Adult) and 3: Neo and Adult combined (Combi). Neo consists of 4500 seizure FVs and 9000 non-seizure FVs randomly selected from 39 neonatal patients. The number of selected seizure and non-seizure FVs per patient is weighted such that each patient contributes equally to the training dataset. This is accomplished by the random selection of a maximum of 24 seizure FVs per patient per 


\section{Chapter 3}

channel. This results in 2 to 4 minutes "seizure" EEG depending on the number of overlapping epochs. Adult is composed similarly resulting in 8235 seizure and 9000 non seizure FVs. The Combi training data set is the combination of $\mathrm{NeO}$ and Adult resulting in 12735 seizure and 18000 non-seizure FVs. Since random sampling of training data may have an effect on classifier performance, 10 Monte Carlo simulations of the complete training and testing procedure are performed. In this way performance measure robustness and its variance is evaluated. Each Monte Carlo simulation runs with a new random selected set of training FVs. Final classifier performance metrics are reported as the average of all 10 Monte Carlo simulations. Training and classification is performed using "leave one patient out" cross-validation (LOO) meaning that in each LOO run an SVM classifier is trained on all but one patient's data. This classifier is then used to classify the left out patient's data. In this way no information from the test patient is taken into account by the training algorithm, resulting in non-biased results. It is evident that when a classifier trained on the Neo data set is evaluated using the Adult data set and vice versa, LOO is not applicable. Hence, only a single classifier is trained on one data set and its performance evaluated using the other data set.

\section{SVM classifier training}

The classification algorithm described in this paper has been developed at the Maastricht University hospital MUMC+ [17]. It is an improved version of the algorithm introduced to the field of neonatal seizure detection by Temko et al [12], which is based on SVM. The improvements consist of patient specific EEG feature baseline correction (FBC) and classifier output post-processing using a Kalman filter. A more detailed description can be found in our recent paper [17]. An SVM is a discriminative model which uses a subset of the training data to construct a surface that separates seizure from nonseizure feature vectors $[25,26]$. Using a Gaussian kernel, the data is 
transformed from the original $\mathrm{N}$ (number of epochs) by $\mathrm{M}$ (number of features, $N>M$ ) dimensional feature space into a higher $\mathrm{N}$ by $\mathrm{N}$ dimensional space where a complex classification problem can be solved with linear discrimination functions. In this higher dimensional space each epoch is characterized by N 'new features'. Owing to the nature of the Gaussian kernel, each new feature equates to a similarity score between two epochs. Only the feature vectors that are close to the decision surface are used by the SVM. In the training dataset each feature is normalized by subtracting the mean and dividing by the standard deviation to ensure that each feature has contributes equally to the model. The test data is normalized with the normalizing parameters from the training set. Optimal SVM classifier parameters $C$ (box constraint) and $\sigma$ (Gaussian spread parameter) are identified using a grid-search for each train-test scenario. Without FBC, optimal combinations of $C$ and $\sigma$ were scattered over the grid. However, for FBC classification, the grid-searches did result in a more restricted range of optimal $C$ and $\sigma$ values. Therefore, these optimal $C$ and $\sigma$ values found for FBC classification were also used for noFBC classification (Table 2). Differences in performance were relatively small and statistically not significant for a range of different $C(10-40)$ and $\sigma(5-8)$ values.

\section{Classifier output post-processing}

Post processing is applied to the classifier outputs and consists of several steps. Each EEG epoch is represented by a feature vector for each channel. The SVM classifier calculates an output for each feature vector. This output represents the signed distance from the decision surface. For each epoch the outputs are then sorted in ascending order. The sorted output time series are subsequently filtered with a Kalman filter to remove random noise. The filtered and sorted output is finally compared to a threshold to make the final classification decision. 
Chapter 3

\section{Performance evaluation}

Seizure detection performance is evaluated in two ways: epoch based metrics and event based metrics. In case of epoch based metrics each epoch is treated as an individual observation which is classified as either seizure or non-seizure. In the testing stage the SVM classifier is applied separately to each EEG channel. A multi-channel score is obtained by simply selecting the highest SVM classifier output of all channels. This multi-channel score is then compared to a detection threshold to obtain a binary decision: 0 - non-seizure; 1 - seizure. This is equivalent to requiring seizure detection in at least one EEG channel. To obtain receiver operating characteristics (ROC) curves, sensitivity is plotted versus specificity at all possible detection thresholds [27]. The area under this curve (AUC) is used to quantify the classification performance of a system and has a value of 0.5 for random classification and 1 for perfect classification. ROC curves of the 10 Monte Carlo simulations are combined using vertical averaging which means that 10 sensitivity values are averaged at fixed specificity values [27]. Another important factor among patients to take into account, are differences in number and duration of seizures. Because AUC values are calculated for the complete dataset, it is important that final performance values are not skewed due to patients with more/or longer lasting seizures. This in particular because longer lasting seizures are usually more easy to detect [28]. To prevent the results to be skewed towards patients with more and longer seizures, the dataset was balanced prior to the calculation of the AUC values. Let Nmax-S and Nmax-NS denote the number of epochs of the patient with the largest number of seizure and non-seizure epochs, respectively. A balanced dataset was achieved by randomly taking multiple copies, Nmax-S and Nmax-NS, of seizure and non-seizure epochs respectively per patient. As a result, each patient was weighted equally in the AUC is calculation. Relative classification performance differences between FBC and noFBC are calculated as a percentage of the maximally achievable gain 
defined as $100 *(F B C-n o F B C) /(1-n o F B C)$. For example an increase in AUC from 0.8 to 0.9 corresponds to $50 \%$ relative increase.

Classification performance can also be quantified by so-called eventbased metrics i.e. 'false detection per hour' (Fd\h) and 'seizure detection rate' (SDR). These metrics are clinically more relevant because the final job of an automated seizure detection algorithm is to alarm only when a seizure is detected. Whether only part of a seizure is detected is therefore of secondary importance. A false detection is defined as a single epoch or continuous segment of epochs classified as seizure without overlap with an actual annotated seizure. A seizure is detected when at least one of its epochs is classified as such.

\section{Statistical analysis}

To apply adequate statistical testing, performance metric distributions generated by the Monte Carlo simulations are first checked for normality using the Shapiro-Wilk test as well as visual inspection [29]. In case of normal distributions a two sample t-test is used, otherwise a Wilcoxon rank sum test is used to compare classifier performance differences. P-values below 0.05 are considered statistically significant.

\section{Results}

Seizure detection performance of three SVM classifiers is evaluated with and without FBC on two test datasets: neonatal EEG and adult EEG. The average AUC values with and without $F B C$ are shown in Table 2. Several observations can be made when the different classifier and test data combinations are evaluated. First, FBC results in a relative performance increase between $52 \%$ and $59 \%$. Because of this distinct with and without $\mathrm{FBC}$ difference between classifier performance, only classification results with $\mathrm{FBC}$ will be presented next.

The average ROC curves (sensitivity versus 1-specificity) for the three classifiers are shown in Fig 14 . The ROC curves of the neonatal test set 


\section{Chapter 3}

(Fig.14B) were skewed towards higher specificity. This indicates that for neonatal seizure detection, classification of seizure epochs is more difficult compared to non-seizure epochs. This was not the case for adult seizure detection where the ROC curves were symmetric around the diagonal line $(01,10)$.
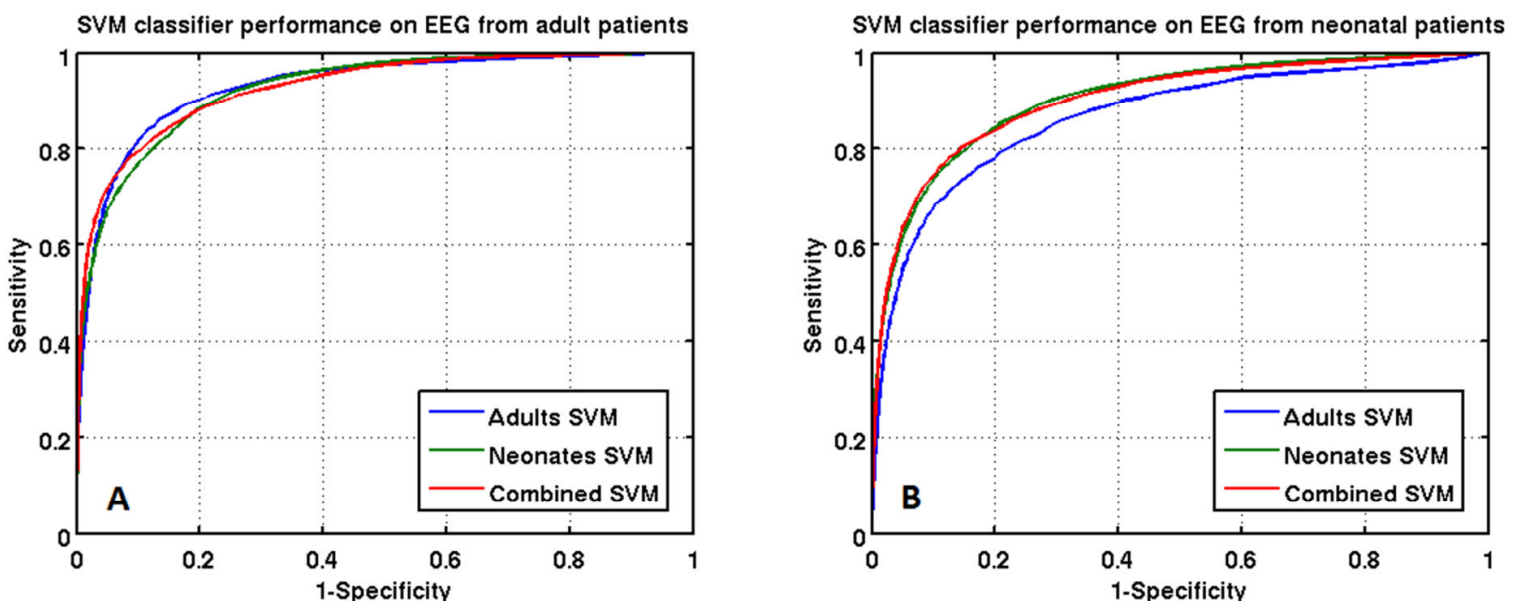

Figure 14 ROC curves for adult seizure detection $(A)$ and neonatal seizure detection (B) In addition to the epoch based metrics, the event based metric FD $\backslash \mathrm{h}$ is provided. The $F D \backslash h$ distributions corresponding to a sensitivity of $80 \%$ are shown in Fig 2.
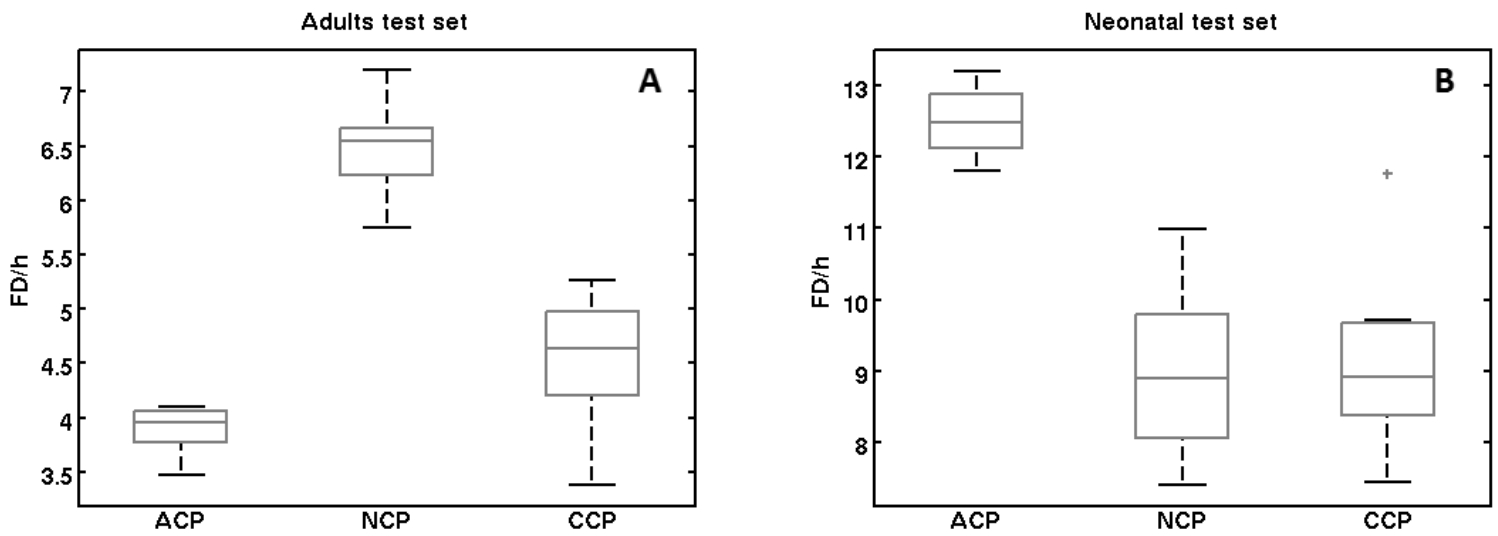

Figure $15 \mathrm{FD} \backslash \mathrm{h}$ distributions belonging to a sensitivity of $80 \%$ for adult seizure detection (A) and neonatal seizure detection (B). 
The results show that neonatal seizure detection is optimal using either the neonatal SVM classifier or the combined classifier. This follows from the significantly higher AUC values (Table 3) and significantly lower number of FDh (Fig. 15B) are found compared to the adult classifier. For adult seizure detection the results were less unambiguous as shown in Fig. 14 and Table 2. Based on the AUC values, all 3 classifiers performed equally well. However, in Fig. 14A it can be seen that the 3 ROC curves intersect. If high specificity is desired, the combined classifier performed best. If high sensitivity is desired, the adults classifier performed best. Furthermore, in the $60-90 \%$ sensitivity range, NCP was lower compared to ACP and CCP. This lower NCP was also reflected in a higher $\mathrm{Fd} \backslash \mathrm{h}$ rate, as shown in Fig. 16A. In addition to classification performance, the SV composition of the combined classifier is evaluated. SVs are those training feature vectors considered to be most relevant for classification. It is assumed that the larger a subset's percentage of training vectors (neonatal or adult) that become SV, the more diverse information is contained in this subset.

Without FBC both the neonatal (49\%) and the adults (51\%) dataset account for approximately half the number of the SVs. With FBC the amount of neonatal SVs increases to 55\%. When the SVs are evaluated per class (seizure and non-seizure) as well, the high neonatal seizure and lower adults seizure class importance become apparent. Of the neonatal dataset $64 \%$ of the seizure FVs becomes SV, versus only $38 \%$ for the adults FVs. 
Chapter 3

\begin{tabular}{|c|c|c|c|c|}
\hline $\begin{array}{l}\text { A: Adults test } \\
\text { set }\end{array}$ & no FBC & FBC & $\begin{array}{c}\text { performance } \\
\text { increase }\end{array}$ & $\mathrm{C}, \sigma$ \\
\hline ACP & $0.85 \pm 0.005$ & $0.93 \pm 0.002$ & $52 \%$ & 40,7 \\
\hline NCP & $0.84 \pm 0.014$ & $0.92 \pm 0.005$ & $52 \%$ & 40,5 \\
\hline CCP & $0.83 \pm 0.04$ & $0.93 \pm 0.009$ & $57 \%$ & 50,6 \\
\hline $\begin{array}{l}\text { B: Neonatal test } \\
\text { set }\end{array}$ & no FBC & FBC & $\begin{array}{l}\text { performance } \\
\text { increase }\end{array}$ & $C, \sigma$ \\
\hline ACP & $0.70 \pm 0.004 * \wedge$ & $0.86 \pm 0.002 * \wedge$ & $54 \%$ & 20,8 \\
\hline NCP & $0.76 \pm 0.042 *$ & $0.90 \pm 0.009 *$ & $59 \%$ & 20,8 \\
\hline CCP & $0.78 \pm 0.028^{\wedge}$ & $0.90 \pm 0.009^{\wedge}$ & $53 \%$ & 50,6 \\
\hline
\end{tabular}

Table 3 Classifier performance evaluated on the Adults data set $(A)$ and the Neo data set (B), with and without FBC. Performance is expressed as average ROC AUC value and its standard deviation. The relative increase in performance due to $\mathrm{FBC}$ is expressed as percentage of the maximal achievable performance gain. SVM parameters $\mathrm{C}$ and $\sigma$ were identified using a grid-search for each train-test scenario. Bold values indicate which classifier results in optimal seizure detection performance. Superscript symbols indicate statistically significant differences $(p<0.05)$ between classifiers (within a column in each table) and do not apply to differences between no FBC and FBC.

* adult classifier performance (ACP) versus neonatal classifier performance (NCP);

^ ACP versus combined classifier performance (CCP);

"ACP versus $C C P$ 
Optimal training dataset composition

\section{Discussion}

\section{Adult versus neonatal seizure detection}

As reviewed by Ramgopal [14] scientific research mainly focuses on different EEG classification methods and features used to detect epileptic seizures. Recent research has focused on feature normalization techniques for age independent seizure detection [17, 22]. However, little attention has been given to methods for optimal training dataset selection with respect to age independent seizure detection [15].

This study shows that an age-independent SVM seizure detection system can successfully be used for seizure detection in both adult and neonatal patients. Despite a significant performance increase when applying FBC, neonatal seizure detection was still sub-optimal using a classifier trained on adult EEG data. The same holds for adult seizure detection using a classifier trained on neonatal EEG data. However, differences in adult seizure detection performance, between the 3 classifiers, were much smaller compared to differences in neonatal seizure detection performance. Although AUC values for NCP (0.92), ACP (0.93) and CCP (0.93) did not differ significantly for the adult test set, FD \h was significantly lower for ACP compared to NCP and CCP. However, the metric FD $\backslash$ h should be approached with caution because it does not take into account the duration of each false detection [28]. Overall, in terms of both AUC values and FD h, neonatal seizure detection performance was lower compared to adult seizure detection performance. Furthermore, we observed that when an SVM classifier was trained on both EEG data from adult and neonatal patients, more neonatal feature vectors become support vector as compared to adult feature vectors. These findings are in support of neonatal seizure detection being a more difficult task compared to adult seizure detection. This underlines the importance of including EEG data in the training data from patients with age matched to the age of the test patients, especially when used for neonatal seizure detection. 


\section{Chapter 3}

As a primary performance measure, the epoch based area under the ROC curve was used. Monte Carlo simulation of the random training data selection provides insight into the variation of the AUC performance measures. In light of the relatively small gains in improving seizure detection, AUC variations caused by random training data set selection can be quite significant. By using Monte Carlo simulations, variations are averaged out resulting in more robust performance measures. Without Monte Carlo simulations one might risk drawing conclusions based on random chance. We therefore strongly recommend to quantify and to minimize variance in performance metrics when developing and evaluating seizure detection methods. Another relevant aspect is the fact that the number and/or duration of seizures among patients may differ considerable. For this reason, we corrected our results to be not skewed towards patients with more and longer duration seizures. Based on our study, we strongly recommend that with regard to seizure detection performance evaluation such a correction procedure must be applied.

\section{Implications and limitations}

Our work shows the importance of training dataset selection for age independent seizure detection in EEG. In a data driven classification approach such as SVM an EEG dataset is both used to develop and test a classification method. The role the training dataset plays in the eventual classifier performance is often neglected. As a result it is very difficult to compare different classification methods trained on different datasets even when evaluated on the same test set. Since the availability of seizure annotated EEG registrations is often a limiting factor in the development of seizure detection algorithms it is important to know which EEG data is most valuable for classifier training. Our results show that FBC enables accurate age independent seizure detection and underline the importance of neonatal seizure EEG for optimal classifier training. In this study we have analysed a large 
dataset of 78 patients (adult and neonatal) with a total of 592 seizures. A limitation lays in the relatively short ( 20 minute) EEG registrations each containing minimally one seizure. This might not reflect a real intensive care setting where a patient is monitored for several days.

Based on the results of this study, our future research will address several matters to further improve (longterm) seizure detection. The SVM-FBC classifier trained for neonatal seizure detection will be tested on a large dataset $(N=53)$ consisting of multiple day EEG registrations from adult intensive care patients as part of a neuromonitoring study. Slow changes in the EEG due to medication, level of awareness and clinical status might influence detection performance. Indeed, feature differences between patients are of similar magnitude to those within a patient over time [22]. It therefore goes without saying that using FBC with a fixed baseline is not optimal for long-term monitoring. To correct for EEG baseline variations our future research will be focussed on the development of an automated baseline-update algorithm. Moreover, it is possible to incorporate more or less online patient specific information into the detection algorithm during long-term monitoring. When a seizure is detected it can be added to the original training set and used to train a new, more patient-specific, classifier. Such a patientspecific classifier can be trained to detect patient-specific seizures but could also be trained to detect patient-specific false detections caused by for example periodic discharges [30]. Eventually, it might be possible to replace the non-patient-specific classifier by a patient-specific one as more information about a patient becomes available during a monitoring session. Moving from patient independent towards patientspecific seizure detection during a monitoring session will also be part of our further research. 
Chapter 3

\begin{tabular}{|c|c|c|c|c|c|}
\hline Patient \# & Age (years) & $\begin{array}{l}\text { Number of } \\
\text { seizures }\end{array}$ & $\begin{array}{l}\text { Minimal } \\
\text { seizure } \\
\text { duration } \\
\text { (mm:ss) }\end{array}$ & $\begin{array}{l}\text { Maximal } \\
\text { seizure } \\
\text { duration } \\
\text { (mm:ss) }\end{array}$ & $\begin{array}{l}\text { Registration } \\
\text { length } \\
\text { (hh:mm:ss) }\end{array}$ \\
\hline 1 & 68 & 12 & $00: 40$ & $02: 55$ & $00: 21: 25$ \\
\hline 2 & 26 & 3 & $02: 00$ & $03: 20$ & 00:20:05 \\
\hline 3 & 32 & 4 & $00: 15$ & 02:05 & $00: 19: 25$ \\
\hline 4 & 60 & 17 & $00: 55$ & $02: 50$ & $00: 34: 25$ \\
\hline 5 & 56 & 7 & $00: 25$ & $00: 55$ & $00: 47: 35$ \\
\hline 6 & 47 & 1 & $02: 10$ & $02: 10$ & $00: 23: 55$ \\
\hline 7 & 83 & 4 & 01:10 & $02: 10$ & $00: 43: 25$ \\
\hline 8 & 75 & 13 & 01:05 & 03:00 & $00: 41: 25$ \\
\hline 9 & 22 & 2 & $04: 20$ & 08:15 & $00: 26: 55$ \\
\hline 10 & 57 & 4 & $00: 45$ & $01: 15$ & $00: 16: 25$ \\
\hline 11 & 65 & 3 & $00: 50$ & $01: 30$ & $00: 26: 35$ \\
\hline 12 & 65 & 5 & 01:00 & 03:10 & $00: 38: 55$ \\
\hline 13 & 41 & 24 & $00: 45$ & $10: 15$ & $02: 15: 15$ \\
\hline 14 & 46 & 1 & $00: 45$ & $00: 45$ & $00: 19: 15$ \\
\hline 15 & 75 & 19 & $00: 15$ & 01:00 & $00: 29: 15$ \\
\hline 16 & 40 & 12 & $00: 45$ & 03:30 & $00: 27: 15$ \\
\hline 17 & 49 & 19 & $00: 40$ & 03:05 & 01:33:35 \\
\hline 18 & 50 & 2 & $02: 10$ & $02: 30$ & $00: 20: 15$ \\
\hline 19 & 39 & 2 & $00: 45$ & $02: 00$ & $00: 23: 15$ \\
\hline 20 & 66 & 2 & $02: 50$ & 03:05 & $00: 24: 45$ \\
\hline 21 & 29 & 7 & $00: 10$ & $01: 30$ & $00: 46: 05$ \\
\hline 22 & 65 & 3 & 01:35 & $02: 15$ & $00: 32: 35$ \\
\hline 23 & 43 & 19 & $00: 10$ & $00: 40$ & $00: 19: 15$ \\
\hline 24 & 37 & 2 & $00: 15$ & 01:45 & $00: 26: 45$ \\
\hline 25 & 70 & 2 & $02: 35$ & $02: 45$ & $00: 43: 15$ \\
\hline 26 & 84 & 16 & $00: 20$ & $01: 00$ & $00: 38: 25$ \\
\hline 27 & 71 & 4 & $00: 30$ & $02: 20$ & $00: 21: 15$ \\
\hline 28 & 88 & 7 & $00: 35$ & 02:05 & $00: 26: 25$ \\
\hline 29 & 79 & 1 & $01: 30$ & $01: 30$ & $00: 21: 05$ \\
\hline 30 & 29 & 3 & $00: 10$ & $00: 10$ & $00: 25: 55$ \\
\hline 31 & 59 & 1 & 01:10 & 01:10 & $00: 31: 45$ \\
\hline 32 & 83 & 6 & $00: 15$ & $00: 25$ & $00: 17: 55$ \\
\hline 33 & 46 & 7 & $00: 20$ & $02: 00$ & $00: 26: 25$ \\
\hline 34 & 23 & 5 & $00: 25$ & $00: 35$ & $00: 29: 55$ \\
\hline 35 & 46 & 1 & $02: 00$ & $02: 00$ & $00: 42: 15$ \\
\hline 36 & 50 & 2 & $00: 10$ & $00: 10$ & $00: 20: 05$ \\
\hline 37 & 27 & 2 & $00: 11$ & $00: 12$ & $00: 20: 45$ \\
\hline 38 & 68 & 2 & $02: 45$ & 03:05 & $00: 27: 25$ \\
\hline 39 & 61 & 4 & 01:05 & 01:45 & $00: 19: 45$ \\
\hline
\end{tabular}

Table 4 Patient information of the Adult dataset

64 
Optimal training dataset composition

\begin{tabular}{|c|c|c|c|c|c|}
\hline $\begin{array}{c}\text { Patient } \\
\quad \#\end{array}$ & $\begin{array}{c}\text { Post } \\
\text { conceptional } \\
\text { Age (days) }\end{array}$ & $\begin{array}{c}\text { Number } \\
\text { of } \\
\text { seizures }\end{array}$ & $\begin{array}{l}\text { Minimal } \\
\text { seizure } \\
\text { duration } \\
\text { (seconds) }\end{array}$ & $\begin{array}{l}\text { Maximal } \\
\text { seizure } \\
\text { duration } \\
\text { (seconds) }\end{array}$ & $\begin{array}{l}\text { Registration } \\
\text { length }\end{array}$ \\
\hline 1 & 280 & 1 & $01: 14$ & $01: 14$ & $00: 19: 19$ \\
\hline 2 & 245 & 3 & 01:13 & 01:41 & $00: 20: 19$ \\
\hline 3 & 252 & 6 & $00: 12$ & 01:04 & $00: 45: 38$ \\
\hline 4 & 254 & 4 & 01:23 & $04: 29$ & 00:19:09 \\
\hline 5 & 286 & 6 & 01:34 & $03: 58$ & $00: 47: 38$ \\
\hline 6 & 245 & 1 & $02: 56$ & $02: 56$ & $00: 19: 49$ \\
\hline 7 & 281 & 9 & $00: 28$ & 01:35 & $00: 19: 39$ \\
\hline 8 & 280 & 7 & 01:12 & 03:11 & $00: 26: 59$ \\
\hline 9 & 282 & 11 & 01:12 & $01: 50$ & $00: 28: 39$ \\
\hline 10 & 238 & 5 & 01:46 & 04:19 & $00: 23: 49$ \\
\hline 11 & 280 & 7 & $00: 15$ & $00: 44$ & $00: 41: 08$ \\
\hline 12 & 281 & 6 & $00: 52$ & 04:00 & 00:32:09 \\
\hline 13 & 280 & 21 & $00: 33$ & $28: 43$ & 02:18:17 \\
\hline 14 & 225 & 12 & $00: 33$ & $11: 38$ & $01: 13: 28$ \\
\hline 15 & 280 & 1 & 01:03 & 01:03 & $00: 26: 09$ \\
\hline 16 & 258 & 5 & $00: 37$ & $02: 17$ & 00:19:09 \\
\hline 17 & 266 & 4 & $01: 50$ & $12: 09$ & $00: 32: 29$ \\
\hline 18 & 281 & 11 & $00: 13$ & $00: 31$ & $00: 27: 49$ \\
\hline 19 & 272 & 14 & 01:00 & $02: 34$ & $00: 41: 38$ \\
\hline 20 & 280 & 1 & $02: 50$ & $02: 50$ & 00:29:09 \\
\hline 21 & 283 & 4 & 01:04 & $06: 55$ & $00: 16: 59$ \\
\hline 22 & 283 & 76 & $00: 11$ & 03:05 & $03: 27: 14$ \\
\hline 23 & 356 & 2 & $05: 16$ & $15: 59$ & $00: 24: 59$ \\
\hline 24 & 267 & 12 & $00: 32$ & $01: 22$ & $00: 29: 29$ \\
\hline 25 & 284 & 64 & $00: 29$ & 03:10 & 02:11:38 \\
\hline 26 & 262 & 5 & $02: 13$ & 09:41 & 00:43:08 \\
\hline 27 & 280 & 15 & $00: 31$ & 03:42 & $00: 30: 39$ \\
\hline 28 & 282 & 3 & $01: 21$ & 01:34 & $00: 23: 19$ \\
\hline 29 & $?$ & 3 & 00:31 & $00: 54$ & 00:16:39 \\
\hline 30 & 281 & 1 & $06: 10$ & $06: 10$ & $00: 25: 09$ \\
\hline 31 & $?$ & 1 & $08: 33$ & $08: 33$ & 00:30:09 \\
\hline 32 & 266 & 1 & 01:50 & 01:50 & $00: 20: 49$ \\
\hline 33 & 197 & 2 & 01:40 & 05:08 & $00: 20: 39$ \\
\hline 34 & $?$ & 1 & 00:31 & $00: 31$ & $00: 21: 39$ \\
\hline 35 & 200 & 3 & 00:39 & $01: 22$ & 00:19:39 \\
\hline 36 & $?$ & 2 & $00: 32$ & $01: 13$ & $00: 22: 59$ \\
\hline 37 & 223 & 5 & $01: 13$ & $08: 46$ & 01:06:08 \\
\hline 38 & 259 & 2 & $00: 36$ & $01: 24$ & 00:19:59 \\
\hline 39 & 294 & 5 & $00: 54$ & 01:37 & $00: 50: 19$ \\
\hline
\end{tabular}

Table 5 Patient information of the Neo dataset 



\section{Chapter 4}

\section{Improved epileptic seizure detection combining dynamic feature normalization with EEG novelty detection}

Bogaarts JG, Hilkman DM, Gommer ED, van Kranen-Mastenbroek VH, Reulen JP. Improved epileptic seizure detection combining dynamic feature normalization with EEG novelty detection. Medical \& biological engineering \& computing. 2016. 
Chapter 4

\section{Abstract}

Continuous electroencephalographic monitoring of critically ill patients is an established procedure in intensive care units. Seizure detection algorithms, such as support vector machines (SVM), play a prominent role in this procedure. To correct for inter-human differences in EEG characteristics, as well as for intra-human EEG variability over time, dynamic EEG feature normalization is essential. Recently, the median decaying memory (MDM) approach was determined to be the best method of normalization. MDM uses a sliding baseline buffer of EEG epochs to calculate feature normalization constants. However, while this method does include non-seizure EEG epochs, it also includes EEG activity that can have a detrimental effect on the normalization and subsequent seizure detection performance. In this study, EEG data that is to be incorporated into the baseline buffer is automatically selected based on a novelty detection algorithm (Novelty-MDM). Performance of an SVM-based seizure detection framework is evaluated in 17 long-term ICU registrations using the area under the sensitivity-specificity ROC curve. This evaluation compares three different EEG normalization methods, namely a fixed baseline buffer (FB), the median decaying memory (MDM) approach, and our novelty median decaying memory (Novelty-MDM) method. It is demonstrated that MDM did not improve overall performance compared to FB $(p<0.27)$, partly because seizure like episodes were included in the baseline. More importantly, NoveltyMDM significantly outperforms both FB $(p=0.015)$ and MDM $(p=0.0065)$. 
Dynamic feature normalization

\section{Introduction}

Nowadays, continuous electroencephalographic monitoring (cEEG) of critically ill patients is an established procedure in intensive care units (ICU). Quantitative EEG (qEEG) allows many hours of EEG data to be compressed onto a graph, greatly reducing the amount of time necessary to detect seizures and transient EEG changes (6). Up to $48 \%$ of ICU patients experience non-convulsive seizures (NCS), much more than would be detected by clinical observation alone $(2,25,26,61,62)$. If those seizures are not detected, and treatment is thus not offered, those patients may suffer brain damage. Therefore, automated seizure detection methods based on qEEG would be an important addition to the $C E E G$ procedure. In past years, numerous seizure detection methods have been developed $(42,45-49,63)$. Research has mainly focused on two aspects of automatic seizure detection: EEG feature computation and methodological aspects of classification (63). Due to time-varying EEG dynamics and high variability in EEG characteristics between patients, reliable automated seizure detection is difficult (64). Because of this, feature normalization is essential for patient-independent epileptic seizure detection. Some research has evaluated the influence of non-stationary EEG background activity on seizure detection $(65,66)$. We recently introduced a feature baseline correction (FBC) procedure which reduces inter-patient variability by correcting for differences in background EEG characteristics (66). FBC is a feature-normalization method based on visual inspection wherein a seizure-free EEG segment is selected at the start of a monitoring session. However, during longterm CEEG, background EEG changes and differences between different patients can be of an equivalent magnitude. Therefore, feature normalization should be applied in a dynamic time-dependent manner (65). EEG baseline variation may be due to circadian rhythm, changes in the state of the patient's vigilance, a response to medication, or changes in EEG recording quality, e.g. changing electrode-tissue impedances $(64,67)$. For optimal FBC functioning, the non-seizure EEG 


\section{Chapter 4}

baseline segment needs to be updated to adapt to changes in the background EEG. Logesparan et al. (65) showed that a normalization procedure based on median decaying memory (MDM) might be a promising normalization method. It computes a feature normalization factor $N_{F}$, based on an on-going unsupervised update of the baseline EEG buffer. However, during long-term cEEG, artefacts, short-duration epileptiform events, and multiple successive seizures can be numerous. Because if this, unsupervised baseline updated might result in corrupt $\mathrm{N}_{\mathrm{F}}$ calculation and by that, hamper seizure detection performance. This raises the question of whether unsupervised MDM is robust enough when cEEG conditions are less than optimal. Our hypothesis is that a semi-supervised baseline update may significantly improve MDM performance. The improvement our MDM approach offers is based upon our method's ability to automatically reject EEG epochs from the baseline buffer. To accomplish this, we implement a novelty detection algorithm. Novelty detection classifies test data that differs in some respect from data available during training (68). A novelty detector trained on the current baseline segment is used to detect 'Novel' epochs. These epochs differ significantly from the current baseline epochs and thus most likely contain artefacts or EEG patterns not similar to actual baseline EEG activity. Consequently, only epochs classified as 'Non-Novel' are used to update the baseline buffer. With this updated baseline buffer, the feature normalization factor and novelty detector are updated. Our major aim is to evaluate the effect of MDM feature normalization, with and without 'Novelty Detection', on Support Vector Machine (SVM) based seizure detection performance. Seizure detection performance is evaluated in terms of the area under the receiver operator characteristics (ROC) curve. To complete our study, the standard fixed baseline method (66) is compared to MDM and Novelty-MDM. 
Dynamic feature normalization

\section{Materials and Methods}

\section{EEG test dataset}

The test dataset consists of 53 cEEG registrations recorded as part of an ongoing ICU monitoring study. At our hospital's general ICU, patients in a comatose state due to central neurological damage (GSC $<=8$ ) were prospectively enrolled in a non-blinded, non-randomized observational study between January, 2011 and March, 2014. This study was approved by our hospital's ethical committee. With informed consent, and permission from each patient's legal representatives, CEEG was performed. To be included in this study, patients had to have been admitted to the ICU, be 18 years of age or older, have central neurological damage and be in a coma as defined by a Glasgow coma score $(G C S)<=8)$, and EEG electrode placement had to be feasible. Patients were selected after consulting the neurologist or neurosurgeon responsible for their treatment. During their EEG registration, 17 out of the 53 patients experienced convulsive and/or non-convulsive seizures due to various etiologies (Table 6). The total number of seizures in the dataset was 1362 and varied per patient from 1 to 384 with a median number of 49 seizures. The minimal duration of a seizure to be annotated as such was 10 seconds, as is recommended by the International Federation of Clinical Neurophysiology (54). The total duration of EEG registration was 4018 hours (median duration: 66 hours, range: 5 - 210 hours). EEG registration was stopped as soon as GCS dropped below 8. EEG electrode configuration was done according to the international 10-20 electrode configuration system, with 19 active electrodes. Signals were processed in the common average derivation. Seizure detection was performed retrospectively while simulating online detection. 
Chapter 4

\begin{tabular}{|c|c|c|c|c|}
\hline Patient \# & Gender & Age & $\begin{array}{l}\text { Monitoring } \\
\text { duration (h) }\end{array}$ & Etiology \\
\hline ICM0006 & Female & 29 & 101.00 & $\begin{array}{l}\text { Not waking after surgery (aortic } \\
\text { surgery) }\end{array}$ \\
\hline ICM0007 & Male & 65 & 41.25 & aortic rupture \\
\hline ICM0013 & Female & 57 & 52.00 & Subarachnoid haemorrhage \\
\hline ICM0015 & Male & 80 & 97.50 & Trauma with subdural haematoma \\
\hline ICM0016 & Male & 69 & 142.00 & Trauma with subdural haematoma \\
\hline ICM0019 & Male & 75 & 76.25 & Postanoxic encephalopathy \\
\hline ICM0021 & Male & 69 & 122.75 & Status epilepticus \\
\hline ICM0022 & Female & 70 & 29.00 & Subarachnoid haemorrhage \\
\hline ICM0028 & Male & 43 & 35.75 & Status epilepticus \\
\hline ICM0030 & Male & 81 & 67.00 & Postanoxic encephalopathy \\
\hline ICM0031 & Male & 66 & 190.00 & Status epilepticus \\
\hline ICM0034 & Female & 69 & 94.75 & Postanoxic encephalopathy \\
\hline ICM0042 & Female & 38 & 345.50 & Status epilepticus \\
\hline ICM0047 & Male & 20 & 236.75 & Status epilepticus \\
\hline ICM0048 & Male & 60 & 40.50 & Trauma \\
\hline ICM0051 & Female & 66 & 168.50 & Postanoxic encephalopathy \\
\hline ICM0053 & Male & 67 & 128.50 & Postanoxic encephalopathy \\
\hline
\end{tabular}

Table 6 Patient characteristics

Feature extraction

EEG recordings were recorded with a sample frequency of $250 \mathrm{~Hz}$, band-pass filtered between 0.5 and $32 \mathrm{~Hz}$ and subsequently downsampled to $25 \mathrm{~Hz}$. Each of the 19 EEG channels was then partitioned into 10 s epochs with a 5 s (50 \%) overlap between epochs. From each epoch in each common-average referenced EEG channel, 103 quantitative features were extracted $(34,35)$. These features stem from different signal description domains such as time, frequency, and information theory and are listed in Table 2 in the previous chapter. Each EEG epoch is now described by a 103 number long feature vector per channel. 
Dynamic feature normalization

Baseline buffer selection

At the start of each monitoring session, an EEG expert selected 3 minutes of artefact-and-seizure-free EEG. This EEG segment served as a baseline buffer and was used to calculate feature baseline values $\left(F_{b s}\right)$. Due to various etiologies, this baseline EEG was allowed to contain nonseizure abnormalities such as periodic discharges, sharp spikes, or a burst-suppression pattern. To restore EEG signal quality, electrode maintenance was often necessary. Because EEG signals registered before electrode restoration can differ markedly from those registered after electrode restoration, a new 3-minute baseline was manually chosen every time electrode maintenance was performed.

\section{MDM based feature baseline update}

The median decaying memory approach (65) is used to update a feature baseline value $F_{b s l}$ for each feature in each channel separately. Updating consists of a weighted average of the median feature value $F$ of the epochs currently in the buffer and the previous value of $F_{b s l}$ :

$F_{b s l}(i)=(1-\lambda) m e d i a n(F(i-1) \cdots F(i-K))+\lambda F_{b s l}(i-1)$ (9)

Equation 1 has two free parameters: buffer size $K$ and $\lambda$. Additional memory beyond the median calculation of the $\mathrm{K}$ epochs is provided by $\lambda$. Optimal results (65) were obtained using a buffer size of $K=236$ and $\lambda=0.99$ corresponding to a memory of several minutes (the effect of a single value decays to $1 \%$ in about 15 minutes for $\lambda=0.99$ ). In our study, features were calculated for 10 second epochs and baseline update was performed every minute. This approach differs from the approach used by Logesparan et al. (65) who used an epoch duration and update interval of both 1 second. To save computational time, a 1 minute update interval was used since feature normalization has to be 


\section{Chapter 4}

computed online for each of the 103 features and for each of the 19 channels. Because of the aforementioned difference in update interval and epoch duration, different values for $\lambda$ and $\mathrm{K}$ had to be used. To match the same original decaying rate, a value of $\lambda=0.72$ had to be used instead of 0.99. Similarly for a buffer size within the optimal range (236 seconds and above), a buffer size of $K=50$ epochs (with 50\% overlap) was used, corresponding to the value 250 used by Logesparan.

Novelty detection using Principal Component Analysis

In contrast to MDM, where $\mathrm{F}_{\mathrm{bsl}}$ is calculated using the 50 most recent epochs (equation 1), Novelty-MDM applies a procedure to select epochs for calculating $F_{b s}$. Instead of the most recent 50 epochs, the 50 most recently selected epochs were used to calculate $F_{b s l}$. Epoch selection was performed by a Novelty detector based on Principal Component Analysis (PCA) (69). This PCA model was trained on the epochs in the actual baseline buffer to detect non-similar epochs.

\section{Principal Component Analysis}

PCA is a statistical procedure that uses an orthogonal transformation $T=X W$ to map a set of feature vectors $\mathbf{X}$ with correlated variables (EEG features) into a set of new uncorrelated variables called principal components (70). These principal components are then sorted so that the first component accounts for the greatest amount of variance, the second component for the second amount of variance, and so on. Dimensionality reduction is performed by retaining only the first $L$ components: $T_{L}=X W_{L}$. The original observations can now be reconstructed using only the first $L$ components: $X_{L}=T_{L} W_{L}^{T}$. The total squared reconstruction error $\mathrm{E}_{\text {rec }}=\left\|X-T_{L} W_{L}^{T}\right\|_{2}^{2}$ can be used as a novelty measure. The idea behind this novelty detector is that the reconstruction error will be small for observations that originate from the same distribution as the observations $X$ used to identify the 
principal components $\mathrm{W}$. Larger reconstruction errors are expected for observations from another distribution.

\section{Novelty-MDM implementation}

Novelty-MDM is performed per EEG channel separately, starting with the manually-selected initial buffer of non-seizure EEG epochs. This buffer is represented by a matrix where each row represents an epoch and each column one of the 103 calculated EEG features. Due to differences in magnitude between different features, each feature is first normalized by subtracting its mean and subsequently dividing it by its standard deviation. Subsequently, the normalized matrix is used to calculate the PCA model. The 'expected reconstruction error' is calculated using a leave-one-out (LOO) procedure. Excluding one epoch at a time, a PCA model is trained on the remaining feature vectors, after which the reconstruction error is calculated for the left-out epoch. This is then repeated for each epoch and the average reconstruction error of this LOO procedure is used to determine a threshold to classify new epochs as similar or novel. If the reconstruction error of a new epoch exceeds $\mathrm{Tr}$ times the expected reconstruction error, the epoch is classified as novel. $\mathrm{Tr}=2$ and $\mathrm{L}=5$ values were chosen heuristically. In case of $\mathrm{Tr}$, the reconstruction error usually ranged between 1 and 2 times the expected reconstruction error. For artifactual epochs, the reconstruction was much larger (in the range of 20-200 or even above). Regarding the number of principal components, inclusion of more than 5 did not relevantly change the calculated reconstruction errors. This is to be expected because the amount of variance each component accounts for decays exponentially. 
Chapter 4

Feature baseline correction

Feature baseline correction (FBC) is an EEG feature normalization method introduced by our group (66) which is performed by subtracting a normalization factor $\mathrm{N}$ from a raw feature value $\mathrm{F}$ :

$F_{N}=F-N$

with

$N=A * F_{b s l}+B$

where $A$ and $B$ are parameters estimated from the training data set. $A$ more detailed description of FBC can be found in our recent paper (66).

Seizure detection framework

The different seizure detection frameworks evaluated in this study are illustrated in figure 16. For each single EEG channel epoch, a set of 103 features is computed. These features are then normalized according to equation 10, after which the normalized feature vector is classified by an SVM classifier as either seizure or non-seizure.

Figure 16A illustrates the use of a fixed baseline segment to calculate $\mathrm{F}_{\mathrm{bs}}$. Figure 16B illustrates MDM, with the trivial exception that feature vectors successfully classified as seizure are excluded from the baseline. The Novelty-MDM approach is illustrated in Figure 16C. With NoveltyMDM, feature vectors classified as non-seizure are subsequently classified by the novelty detector as either "Novel" or "Non-Novel". Only feature vectors classified as "Non-Novel" are incorporated into the baseline buffer. Using the median decaying memory approach, a new set of normalization factors is calculated based on the updated baseline buffer. Based on this updated baseline buffer, a new novelty detector is calculated. The updated normalization factors and novelty detector are then applied to subsequent epochs. 


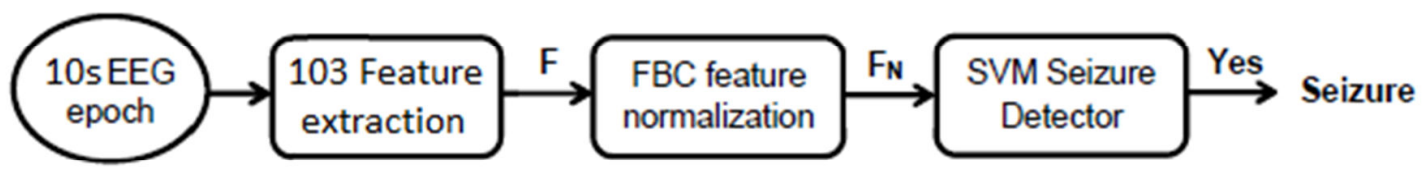

A: Fixed baseline

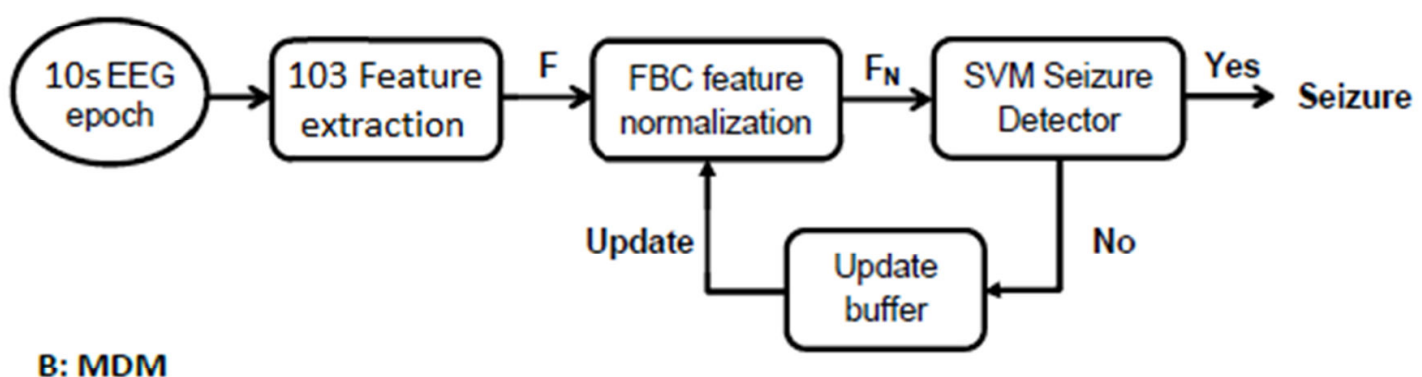

B: MDM

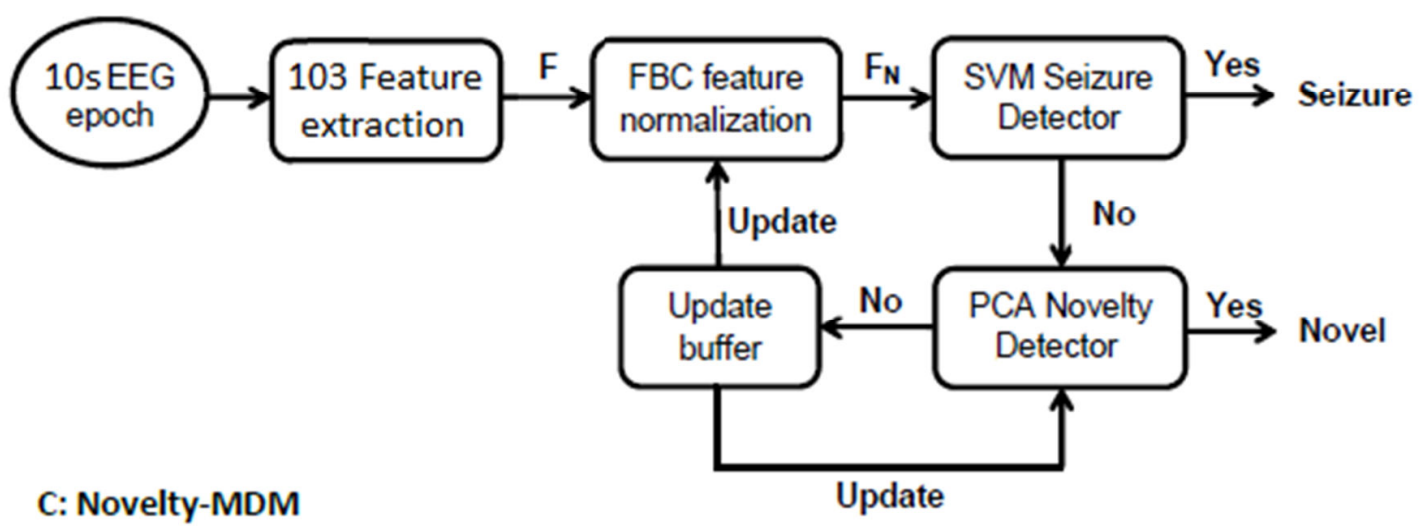

Figure 16 Schematic overview of SVM based seizure detection framework with three different normalization methods. A: normalization constants derived from a fixed and manually selected EEG baseline segment. B: Dynamic feature normalization using MDM. C: Dynamic feature normalization using Novelty-MDM. 
Chapter 4

\section{SVM seizure detection performance evaluation}

The SVM seizure detector was trained on a randomly chosen subset of epochs from a set of routine EEG registrations from 39 neonatal patients. This classifier was readily at our disposal and although it was trained on neonatal EEG, can also successfully be used for seizure detection in adults (71). Because the random selection of the training data set may introduce variance in performance metrics, Monte-Carlo simulations were performed by training 25 classifiers trained on 25 random training subsets. Seizure detection performance was subsequently evaluated for these 25 classifiers using the test EEG dataset of 17 ICU patients. The average and standard deviation of the performance per patient allows for statistical testing, to determine differences between the three seizure detection frameworks. From our ICU dataset, the 17 patients with seizures were used to evaluate seizure detection performance using the 3 different feature normalization methods: Fixed baseline (FB), MDM, and Novelty-MDM.

The seizure detection procedure was applied to each channel separately. If a seizure was detected in at least one channel, the complete epoch was classified as seizure.

To evaluate classifier performance, an ROC curve was obtained per EEG registration by plotting sensitivity versus specificity for all possible detection thresholds (41). The area under this curve (AUC) was then used as a measure of the performance. AUC range is between 0.5 for random and 1 for perfect classification. AUC values were calculated per patient and final performance measures were obtained by taking the average of the 25 AUC values. Group differences between the 3 seizure detection frameworks were tested for statistical significance using the non-parametric Wilcoxon signed-rank. Differences in performance per patient were tested for statistical significance using a paired t-test. Pvalues below 0.05 were considered statistically significant. 
Dynamic feature normalization

\section{Results}

Looking at each individual patient, seizure detection performance was highest for Novelty-MDM in 8/17 patients, highest for MDM in 4/17, and highest for FB in $3 / 17$ patients. For a single patient all three methods resulted in the same performance and for another single patient either FB or Novelty-MDM performed best. The Bland-Altman plots in figure 17 show performance differences between all three methods by plotting method to method performance difference versus their average performance. Specific AUC value distributions for each of the 3 normalization procedures are shown in figure 18. Average AUC values per patient and per normalization method can be found in the appendix.

\section{MDM versus $F B$}

To compare MDM versus FB feature normalization, Figure 17A shows MDM minus FB AUC values versus their mean $((\mathrm{MDM}+\mathrm{FB}) / 2)$ per patient. Ten patients showed improved performance, 5 patients decreased performance and for the remaining 2 patients, no statistically significant difference was found. In the complete group MDM performance was not significantly different from FB ( $p=0.27$, Wilcoxon signed rank test).

Novelty-MDM versus $F B$

Overall, Novelty-MDM was significantly better compared to FB $(p=0.015)$. Figure 17B shows that Novelty-MDM achieved higher performance for 11 patients, lower performance for 4 and equal performance for the remaining two patients. 

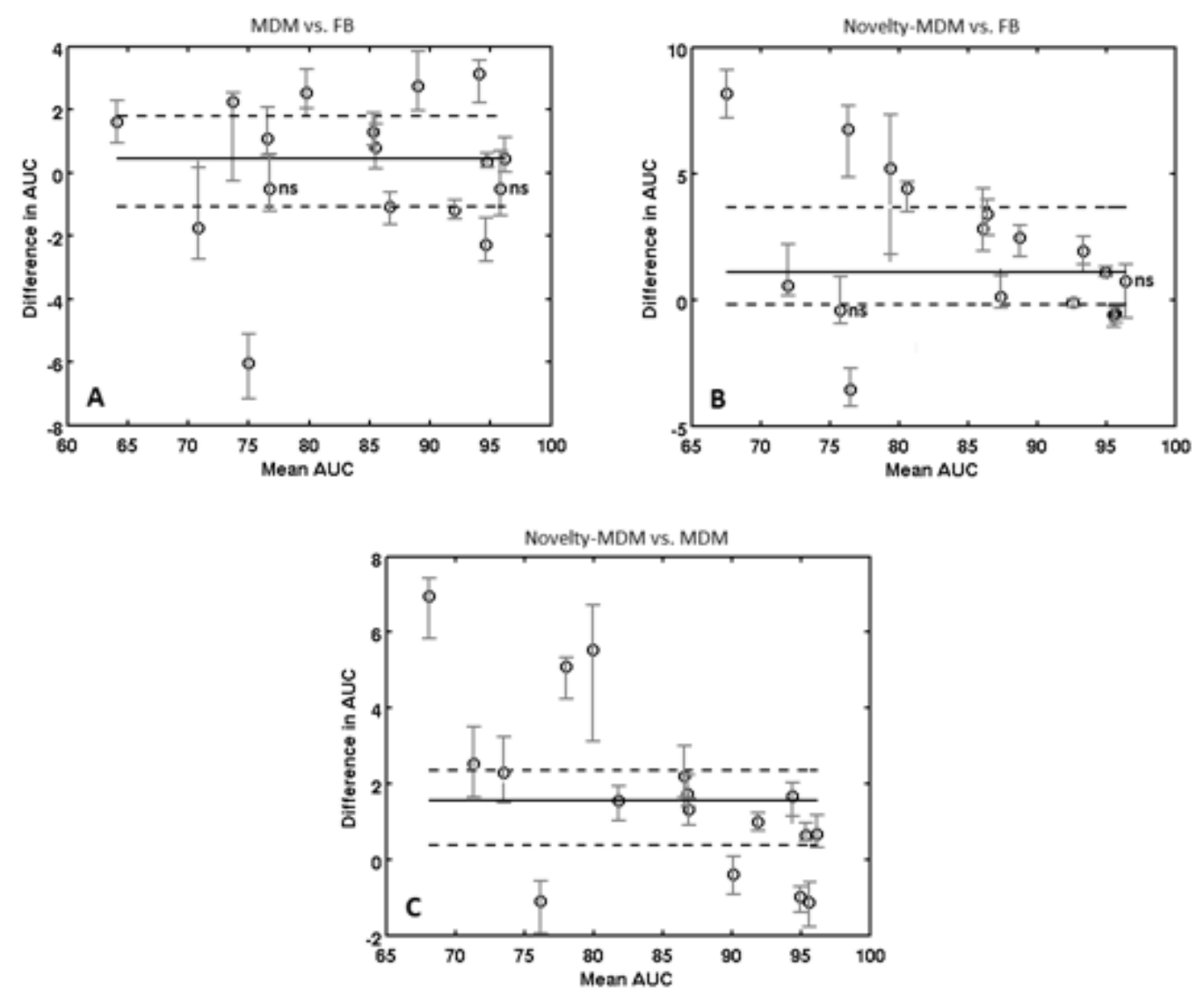

Figure 17 Mean-difference plots of the AUC values per patient. Mean AUC values of two methods are plotted against their difference. Each circle represents the median value of the 25 Monte-Carlo simulations, and each red bars indicate the corresponding 25 and 75 percentile values. Horizontal lines indicate the group median (-), 25 and 75 percentile values (--). Subplot A: group difference MDM vs. FB $(p=0.27)$. Subplot $B$ : group difference Novelty-MDM vs. FB $(p=0.015)$. Subplot $C$ : group difference Novelty-MDM vs. MDM $(p=0.0065)$. Non-statistically differences are indicated with ns. 
Dynamic feature normalization

Novelty-MDM versus MDM

The numbers presented so far showed that both MDM and NoveltyMDM outperform FB. Patient specific Novelty-MDM versus MDM differences in classification performance are shown in Figure 17C. Overall, Novelty-MDM performed better than MDM ( $p=0.0065)$. In 13 patients higher performance was found and lower for the remaining 4 patients. Detailed evaluation of the latter four cases revealed that this lowered performance was due to the presence of periodic epileptiform discharges (PED) and can be explained by the illustration in Figure 19. It shows that a correctly detected seizure was followed by an episode with PED. Novelty-MDM classified more epochs as seizure compared to MDM. This is because at the start of the PED episode, MDM included these epochs into the baseline buffer whereas Novelty-MDM did not. As a result, in case of MDM, the subsequent epochs containing PED were more similar to the baseline, and consequently, more likely to be classified as non-seizure. Novelty-MDM, on the other hand, excluded these epochs with PED because they were marked as 'novel' as can be seen in the bottom plot. As a result, the subsequent PED epochs were less similar to the baseline and consequently became more likely to be classified as seizure.

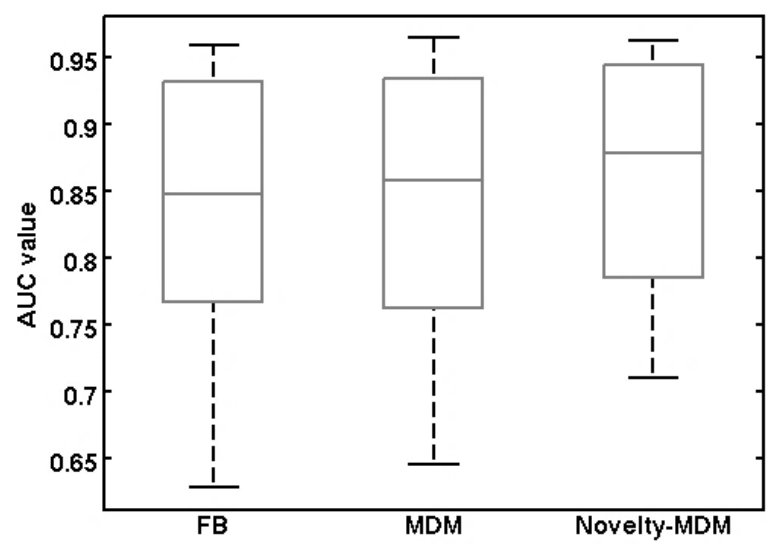

Figure 18 Boxplots describing the AUC value distributions for each normalization method. 
Chapter 4
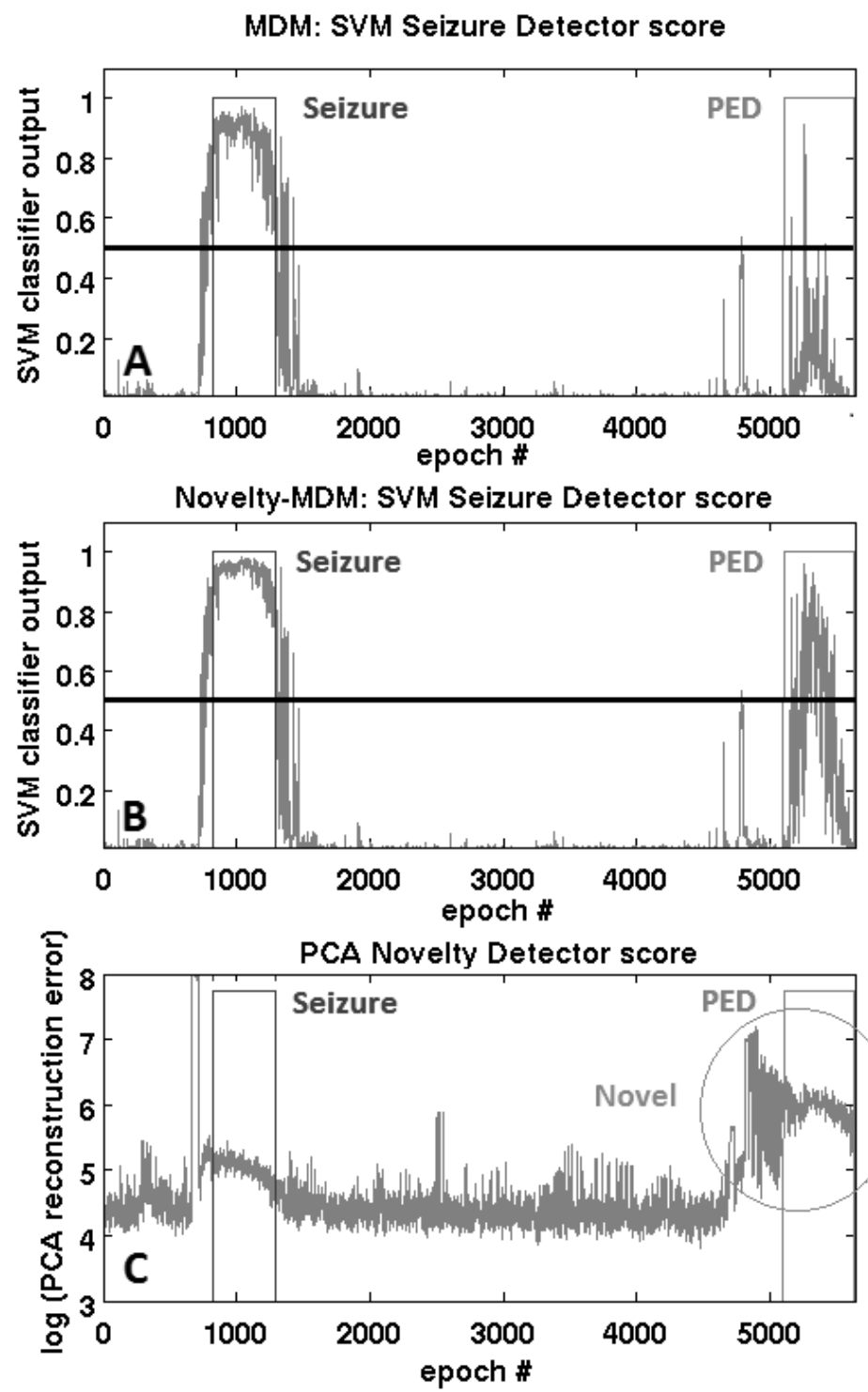

Figure 19 SVM classifier output for MDM (A) and Novelty-MDM (B), a detection threshold of 0.5 was used as indicated by the horizontal lines. The Novelty detection score (reconstruction error) is shown in subplot $C$. The rectangles indicate an episode with seizures and an episode with PED. The circle indicates an episode of epochs that are classified as novel. The first half of this episode contains numerous electrode artefacts and the second half contains PED. Consequently, Novelty-MDM does not update the baseline during this episode because the artifactual and PED epochs were classified as Novel. 
Dynamic feature normalization

\section{Discussion}

\section{Dynamic feature normalization}

To correct for changing EEG characteristics and differences between patients, various feature normalization methods have been evaluated by Logesparan et. al. (65). Best performance was achieved by feature normalization using the median decaying memory (MDM) method. MDM uses the median feature value of a sliding baseline buffer for normalization. However, during long term EEG monitoring, especially in an intensive care unit, seizures, artefacts, epileptiform activity, and other abnormal EEG patterns can be numerous. These patterns might contaminate the baseline buffer and by that, reduce seizure detection performance. Our study provides evidence that MDM is not robust enough for reliable dynamic feature normalization during long-term ICU EEG monitoring. We conclude this from the observation that in our 17 patients, MDM did not result in overall better performance compared to feature normalization with a fixed baseline. However, application of our newly introduced Novelty-MDM approach did result in an overall increase in performance both with regard to $F B$ and MDM normalization. Novelty-MDM is based on a selection procedure to prevent erroneous epochs being included into the baseline buffer thereby improving feature normalization and subsequent seizure detection.

Although Novelty-MDM resulted in better performance for the majority (11/17) of patients, in four patients performance was lower compared to MDM. In three of these four patients, the decrease was due to false detection of epochs containing periodic epileptiform discharges (PED). This finding can be explained by the different behaviour of MDM versus Novelty-MDM. In the presence of PED, whose feature values are between background EEG and seizure EEG activity, MDM includes the PED epochs into the baseline buffer making it more 'seizure like' and 


\section{Chapter 4}

thereby hampering seizure detection. Novelty-MDM on the other hand, rejects these PED epochs and in this way prevents contamination of the 'background EEG' baseline buffer. As a result, MDM becomes less sensitive but more specific in the presence of PED whereas NoveltyMDM becomes more sensitive but less specific. However, the presence of PED does not necessarily result in lower performance for NoveltyMDM. Actually, better performance was found in four other patients (patient 6, 7, 10 and 17). Whether the presence of PED results in higher or lower AUC values might depend on the relative number of seizure/ PED epochs, their occurrence in time relative to each other and the severity of the epileptiform activity i.e. how closely they resemble true seizure activity. This illustrates that our current seizure detection algorithm is not always accurate enough to distinguish between epileptiform and seizure activity. To improve on this, more sophisticated algorithms are needed (72). However, the underlying problem is the fact that seizure detection is approached as a two-class problem instead of a multi-class problem (non-seizure, seizure, epileptiform activity, and possibly other EEG phenomena). A restriction with regard to this will be that even an EEG expert cannot always make a clear distinction between epileptiform and epileptic activity (73). This fundamental issue will always remain and by that limit automated reliable seizure detection.

\section{Implications, limitations and future research}

This paper focused on challenges met in robust dynamic feature normalization applied in automated seizure detection algorithms. So far, MDM feature normalization using a sliding baseline did not take into account the presence of EEG patterns that might corrupt feature normalization. Our results have shown that a non-selective way of baseline update can have a negative effect on classification performance. Baseline epoch selection using a PCA based novelty detector is a candidate solution to this problem. In cases where 
Novelty-MDM resulted in lower performance, it became clear that this was due to epochs containing epileptiform activity. Future research should focus on distinguishing epileptiform EEG from epileptic EEG. In the setting of seizure versus non-seizure classification, detection of epileptiform activity, in particular PED, are considered false detections. However, PED detection could also be considered useful because it is associated with a higher risk of seizures $(13,16)$. Other sources that may cause lower detection performance, apart from electrode failure, muscle and movement artefacts, are patterns that are rhythmic in nature but do not represent seizure activity. Two of our patients had long-lasting periods of frontal intermittent rhythmic delta activity (FIRDA) which were falsely detected as seizure. If such EEG patterns persistently trigger false detections, future improvements incorporating these patterns into the SVM seizure detection algorithm as non-seizure, would be of great value. Moreover, incorporating patient specific seizure information during online monitoring has proven to improve seizure detection performance (74-76). Further research could be of great value when focused on both incorporating various types of patient specific information such as seizure and background EEG data, as well as EEG patterns that caused false detections. 
Chapter 5

From non-patient towards patient specific epileptic seizure detection in comatose patients 
Chapter 5

\section{Abstract}

Continuous electroencephalographic monitoring of critically ill patients is an established procedure in intensive care units. Seizure detection algorithms play a prominent role in this procedure. Objective: improve epileptic seizure detection in comatose patients by incorporating patient specific EEG data into the detection algorithm. Methods: While simulating online seizure detection, good and false detections were added to the non-patient specific training data set. Each time the training data set is updated, a new Support Vector Machine (SVM) classifier is trained and used for subsequent seizure detection. Performance was evaluated in 17 long-term ICU registrations. Results: It is demonstrated that, for equal good detection rates, the number of false detections per hour was significantly lower for patient specificcompared to non-patient specific seizure detection ( 2.2 versus $5.7, p=$ 0.002 , Wilcoxon signed-rank test). Conclusions: Our results showed that significant performance increase can be achieved by incorporating both good and false detections into the SVM classifier. However, when false alarms are triggered by periodic epileptiform discharges, incorporating this EEG data can result in worse performance. Significance: In case no patient specific data is available, seizure detection can be made patient specific and thereby improved, by incorporating good and false detections. 
Patient specific seizure detection

\section{Introduction}

In an intensive care unit (ICU) electroencephalography (EEG) is widely used as an examination and monitoring tool. In this setting the incidence of non-convulsive seizures (NCS) in patients with coma can be up to $48 \%$, which is much higher than suggested by clinical suspicion alone [1-5].

Automated EEG monitoring for epileptic seizures in comatose patients allows for continuous surveillance of brain function, thereby avoiding the time consuming task to be performed by an EEG expert. Compared to patients admitted to a specialised epilepsy monitoring unit, automated seizure detection in ICU patients is much more difficult due to the high variety of complex EEG patterns and abundance of recording artefacts [6, 7]. To address this challenge, sophisticated detection algorithms, for example based on support vector machines (SVM), are used to learn the differences between seizure and non-seizure EEG from many different patients. This learned information is then transformed into a classifier that classifies new EEG samples. Essential for classifier performance is its generalization ability, i.e. to correctly classify diverse unseen data. This in particular in case of long-term monitoring where both non-seizure and seizure EEG may differ between patients and changes over time. Design of a patient-specific classifier might result in better classification performance for monitoring a "new", so far unseen, patient. Our idea of including patient specific information into a classifier to improve seizure detection performance is not completely new. Haas et al. [8] used data from the first available seizure in a registration to adapt their detection algorithm. Thomas et al. [9] adapted a Linear Discriminant Analysis (LDA) model using 'exemplar' patient-specific data. They obtained performance increase for most patients but also a large decrease for some other patients. Shoeb et al. showed that a detector with knowledge of both an individual's seizure and non-seizure EEG outperforms a detector limited 


\section{Chapter 5}

to knowledge of only an individual's non-seizure EEG [10]. The aforementioned methods use patient specific EEG data either available beforehand or selected from the test data itself. However, in clinical practice usually no prior patient-specific EEG is available and it is unknown whether or when a patient will have seizures.

In a previous study we showed that feature baseline correction (FBC) [11] in combination with SVM is a successful first step in making seizure detection more patient specific. FBC was applied on training data to reduce inter-patient variability and improves classifier performance. For classification, FBC takes into account a changing background EEG baseline using a median decaying memory approach in combination with a Novelty Detector [12, 13]. During monitoring, patient specific information becomes available when the SVM classifier marks an EEG segment as 'seizure'. Epochs correctly classified, on the basis of an EEG expert's judgement, as seizure (true positive) are then marked as 'seizure' and false detections (false positive) as 'non-seizure'. In this way expert judgements are used to automatically adapt the SVM classifier. Including false detections as 'non-seizure' might be an adequate way to reduce the number of subsequent false detections caused by EEG artefacts or typical EEG patterns regularly recorded in comatose patients. In particular Periodic Epileptiform Discharges (PED) and frontal intermittent rhythmic delta activity (FIRDA) may cause persistent false detections [7, 12]. PED may complicate accurate seizure detection because, both visually and feature-wise, they often closely resemble seizures. The major aim of this study was to demonstrate how both true and false positive detections can be used to adapt an SVM classifier to the particular patient being monitored. It was hypothesized that inclusion of EEG expert verified detections can significantly increase online seizure detection performance. 
Patient specific seizure detection

Methods

\section{EEG test dataset}

The test dataset consists of 53 cEEG registrations recorded as part of an ongoing ICU monitoring study. At our hospital's general ICU, patients in a comatose state due to central neurological damage (GSC $<=8$ ) were prospectively enrolled in a non-blinded, non-randomized observational study between January, 2011 and March, 2014. This study was approved by our hospital's ethical committee. With informed consent, and permission from each patient's legal representatives, cEEG was performed. To be included in this study, patients had to have been admitted to the ICU, be 18 years of age or older, have central neurological damage and be in a coma (GCS <=8), and EEG electrode placement had to be feasible. Patients were selected after consulting the neurologist or neurosurgeon responsible for their treatment. Seventeen out of the 53 patients experienced convulsive and/or nonconvulsive seizures during the EEG registration due to various etiologies (Table 6, Chapter 4). The total number of seizures in the dataset was 1362 and varied per patient from 1 to 384 with a median number of 49 seizures. The minimal duration of a seizure to be annotated as such was 10 seconds, as is recommended by the International Federation of Clinical Neurophysiology (54). The total duration of EEG registration was 4018 hours (median duration: 66 hours, range: 5 - 210 hours). EEG registration was stopped as soon as GCS dropped below 8. EEG electrode configuration was done according to the international 10-20 electrode configuration system, with 19 active electrodes. Signals were processed in the common average derivation. Seizure detection was performed retrospectively while simulating online detection. 
Chapter 5

\section{Feature extraction}

EEG electrodes were placed according to the $10-20$ system and recordings were acquired with a sample frequency of $250 \mathrm{~Hz}$, band pass filtered between 0.5 and $32 \mathrm{~Hz}$ and subsequently down sampled to 25 $\mathrm{Hz}$. Each of the $19 \mathrm{EEG}$ channel recordings was then partitioned into 10 seconds epochs with 5 seconds (50\%) overlap between epochs. From each epoch in each common-average referenced EEG channel, 103 quantitative features $[14,15]$ are computed. These features stem from different signal description domains such as time, frequency, and information theory and are listed in Table 2 in chapter 3. Per channel, each EEG epoch is now described by a feature vector with length of 103 .

\section{Feature baseline correction}

Feature baseline correction (FBC) is an EEG feature normalization method introduced by our group [11] and is performed by subtraction of a normalization factor $\mathrm{N}$ from a raw feature value $\mathrm{F}$, as described in the previous chapter.

\section{Baseline buffer selection}

At the start of each patient's monitoring session, an EEG expert selects, by visual inspection, 3 minutes of artifact and seizure free EEG. This EEG segment serves as a baseline buffer and is used to calculate feature baseline values (Fbsl). To restore EEG signal quality, electrode maintenance was sometimes necessary. Because EEG signals before and after electrode restoration can be markedly different, a new 3 minute baseline is manually chosen each time electrode maintenance was performed. Epochs of electrode maintenance were easily recognized retrospectively in our recordings since EEG storage was halted resulting in recording gaps. 
Patient specific seizure detection

Median decaying memory based feature baseline update The median decaying memory approach (MDM) [13] is used to update a feature baseline value Fbsl for each feature in each channel separately. Updating consists of a weighted average of the median feature value $F$ of the epochs currently in the buffer and the previous value of Fbsl, as described in the previous chapter.

\section{Novelty-MDM implementation}

To update the feature baseline only with similar epochs, novelty-MDM was performed per EEG channel separately, and started with the manually selected initial buffer of non-seizure EEG epochs. This buffer is represented by a matrix where each row represents an epoch and each column one of the 103 calculated EEG features. Due to magnitude differences between various features, each feature was normalized by subtracting its mean and divided by its standard deviation. The resulting normalized matrix was used to calculate a Principal Component Analysis (PCA) model [16]. The expected reconstruction error of this model was calculated using a leave-one-out (LOO) procedure as follows: excluding one epoch at a time, a PCA model was trained on the remaining feature vectors after which the reconstruction error was calculated for the leftout epoch. The average reconstruction error of this LOO procedure was used to determine a threshold to classify new epochs as similar or novel. If the reconstruction error of a new epoch exceeded Thr times the expected reconstruction error, this epoch was classified as novel. Values $\operatorname{Tr}=2$ and $L=5$ were chosen heuristically. In case of $\operatorname{Tr}$, the reconstruction error usually ranged between 1 and 2 times the expected reconstruction error. For artifactual epochs, the reconstruction was much larger (in the range of 20-200 or even above). In case of the number of principal components, including more than 5 had little influence on the calculated reconstruction errors. This is to be 


\section{Chapter 5}

expected because the amount of variance each component accounts for drops exponentially.

\section{Seizure detection framework}

In this study our patient specific SVM (PS-SVM) is compared to the standard SVM as shown in Figure 20. For each single channel EEG epoch a set of 103 features is computed. These features are then normalized (FBC), after which the normalized feature vector is SVM classified as seizure or non-seizure. The SVM classifier was trained on a randomly selected subset of EEG epochs from routine EEG registrations from 39 neonatal patients. Random selection of training data has shown to introduce variation in classification results [17]. Because of this, a Monte-Carlo simulation was performed by training 10 classifiers trained on 10 random training subsets. Seizure detection performance was subsequently evaluated for these 10 classifiers using the test EEG dataset. The average and standard deviation of the performance per patient allows for statistical testing of differences between the SVM and PS-SVM seizure detection frameworks.

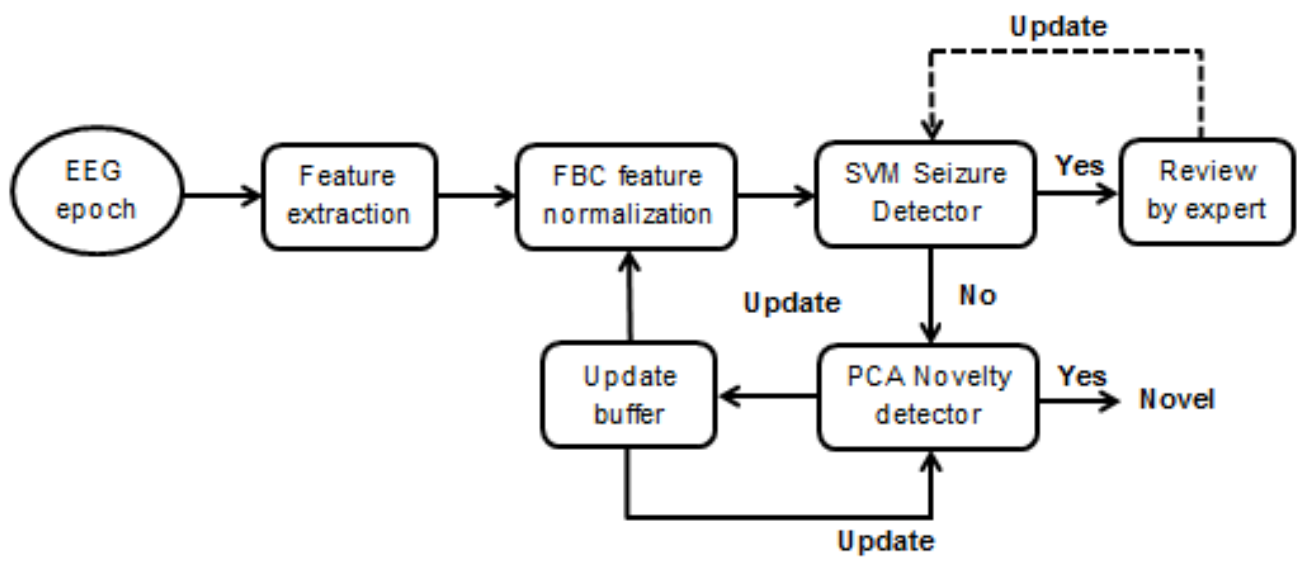

Figure $\mathbf{2 0}$ Schematic overview of SVM based seizure detection framework. For PSSVM, detected epochs are used to update the SVM classifier. SVM and PS-SVM differ in the 'Update' step after EEG expert review (-->). 
Patient specific seizure detection

\section{PS-SVM classifier update}

When adding patient specific information to the standard SVM classifier, it is important to have a training dataset which is balanced in terms of number of epochs per patient and number of epochs per class (seizure and non-seizure). The original training dataset contains 13500 epochs from 39 patients with maximally 400 epochs per patient. Therefore, maximally 400 patient specific epochs (200 seizure and 200 non-seizure) are added to the original training dataset. In case the number of patient specific epochs for a class exceeds 200, only the 200 most recent epochs are used to train the PS-SVM. After a delay of 15 minutes a new SVM classifier is trained that replaces the previous classifier. This delay was chosen to account for the time it takes in a real monitoring setting, to notify an EEG expert and to subsequently review the EEG.

\section{Seizure detection performance evaluation}

SVM classifier output was computed for each single-channel EEG epoch. These outputs were converted to a posterior probability $\mathrm{P}$ using a sigmoid function [18]:

$P($ seizure $\mid f)=\frac{1}{1+\exp (A f+B)}$

where $f$ is the SVM classifier score and $A$ and $B$ are parameters of the sigmoid function. The latter two were estimated from a set of 41 routine adult patient EEG registrations (calibration dataset) [17] using the method described by Platt [19]. This conversion to a posterior probability makes detection threshold selection more intuitive because it now lies on the interval 0-1 [18]. Finally both metrics were averaged over 10 Monte-Carlo simulations. The use of different detection thresholds illustrates the effect on SVM and PS-SVM seizure detection 


\section{Chapter 5}

performance. Recall, defined as the number of true positives divided by the total number of positives (eq.6) better reflects performance in case of an unbalanced distribution of seizure and non-seizure epochs [20]. In addition to sensitivity and recall, the event based metrics false detections per hour (FDH) and good detection rate (GDR) were also computed. GDR is the percentage of correctly detected seizures. If a seizure is detected any time between the start and end of a seizure, this was considered a good detection [21]. A false detection was defined as a series of one or more subsequent detections not overlapping the annotated seizure. Event based metrics are thought to better reflect performance in clinical practice [20]. Since FDH does not take into account the duration of a false detection, also mean false detection duration (MFDD) was calculated [22]. Differences between SVM and PSSVM seizure detection performances were tested for statistical significance using the non-parametric Wilcoxon signed-rank test. Pvalues below 0.05 were considered statistically significant. This test was both used to compare SVM and PS-SVM on a per patient basis as well as for the complete seventeen patient's data set.

\section{Results}

Figure 21 shows the distribution of event-based metrics GDR, FDH and MFDD for SVM and PS-SVM for thresholds between 0.3 and 0.7. Each box represents the distribution of the metrics for 17 patients; the red lines indicate median values. Figure 22 clearly shows that by lowering the detection threshold, GDR increased both for SVM and PS-SVM. In parallel however, FDH and MFDD also increased. Comparison of PS-SVM versus SVM per detection threshold shows that median PS-SVM FDH was significantly lower than SVM FDH for all 5 thresholds ( $p<0.001$ for $T=0.3-0.6$ and $p<0.01$ for $T=0.7$, Wilcoxon signed-rank test). No significant difference between PS-SVM GDR and SVM GDR was found. By varying the detection threshold, median values of GDR and FDH were used to construct receiver operator characteristics (ROC) curves for 
SVM and PS-SVM (Figure 22A). Optimal performance was chosen as the point on the ROC curve closest to ideal classification performance, namely FDH $=0$ and GDR 100\%.
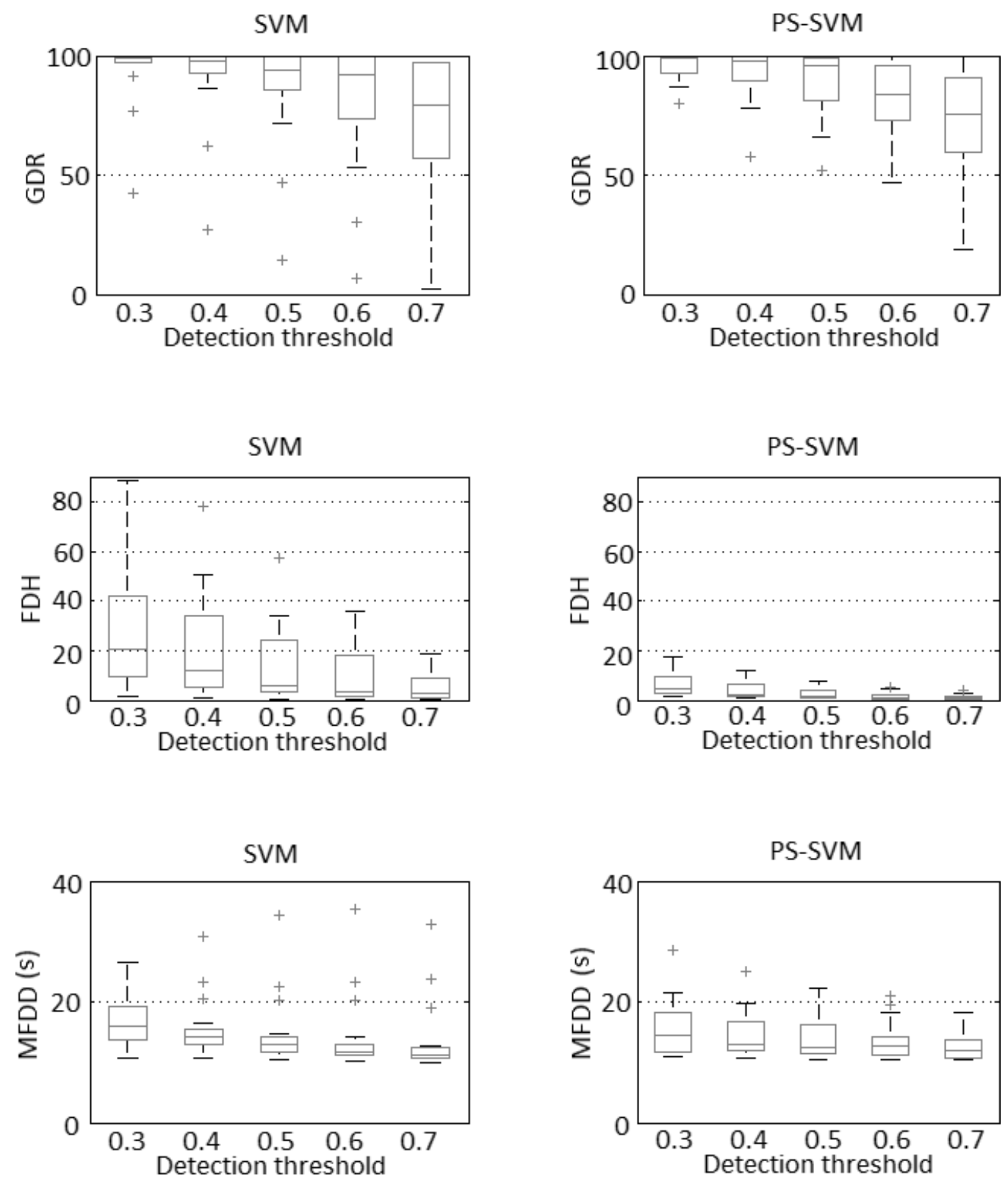

Figure 21 SVM (left) and PS-SVM (right) classifier performance expressed in eventbased metrics GDR (top), FDH (middle) and MFDD (bottom). On each box, the red line mark is the median, the edges of the box are the 25th and 75th percentiles, the whiskers extend to the most extreme data points not considered outliers, and outliers are plotted individually (+). 


\section{Chapter 5}
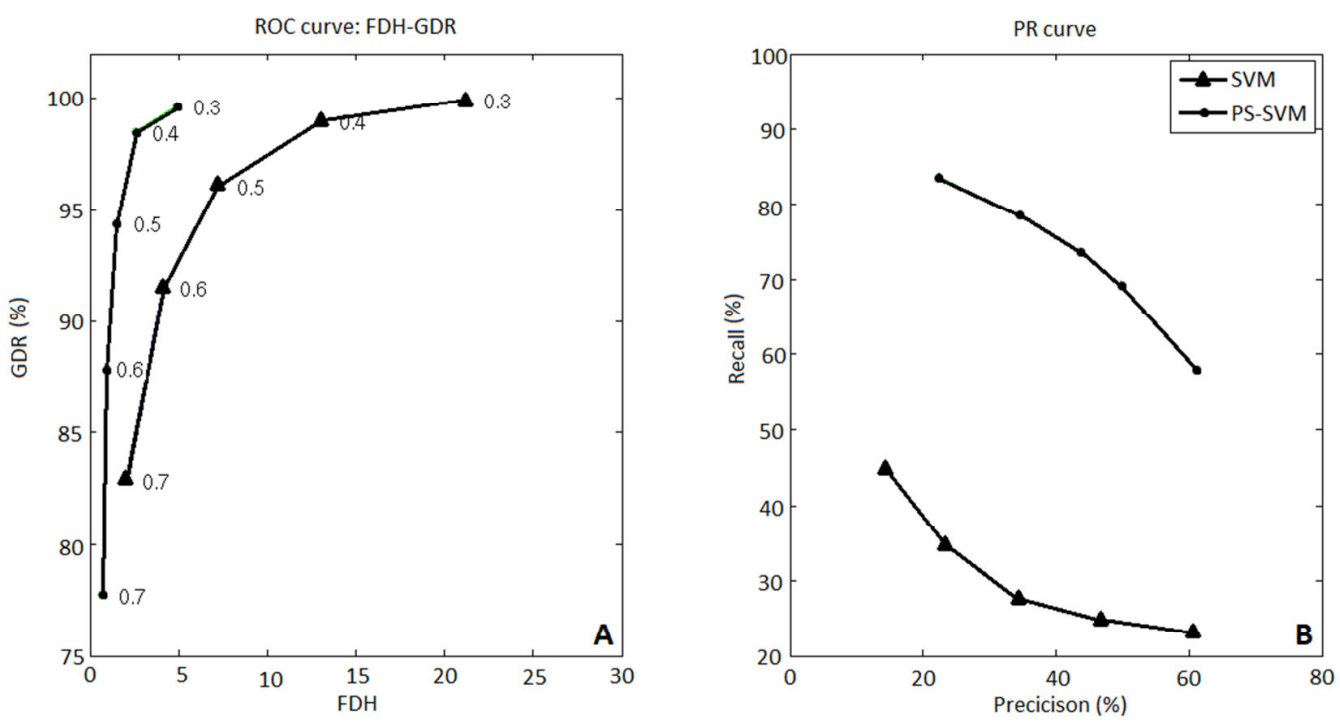

Figure 22 ROC curves based on the event-based metrics GDR versus FDH evaluated at different detection thresholds for SVM (blue) and PS-SVM (green). Each GDR-FDH point label is the specific detection threshold used in computation. B: Summary of epoch based metrics Precision and Recall. Each data point presents the median value of the 17 patients.

As a result, different optimal detection thresholds for SVM (0.5) and PSSVM (0.4) were found. Using these thresholds, median $98 \%$ PS-SVM GDR was not significantly different from $96 \%$ SVM GDR $(p=0.38$, Wilcoxon signed-rank test). However, median PS-SVM FDH was significantly lower than SVM FDH (2.2 versus 5.7, $\mathrm{p}=0.002$, Wilcoxon signed-rank test). Figure 22B shows the epoch based precision-recall curves indicating that both PS-SVM and SVM achieve similar precisions. However, SVM recall was much lower meaning that for each TP epoch more FP epochs were detected. For example, for $60 \%$ precision, $24 \%$ (SVM) and 58\% (PS-SVM) recall means that (using eq. 6) for every true positive, SVM detected 3.2 false positives versus only 0.72 for PS-SVM. Table 7 shows GDR and FDH for SVM and PS-SVM per patient. PS-SVM resulted in a performance increase for 10 patients and in a performance decrease for 2 patients. For the remaining 5 patients, PS-SVM resulted in a decrease in one metric and an increase in the other. 
Patient specific seizure detection

\begin{tabular}{ccccc} 
Patient & \multicolumn{2}{c}{ GDR } & \multicolumn{2}{c}{ FDH } \\
& SVM & PS-SVM & SVM & PS-SVM \\
1 & 0,94 & 0,83 & 3,8 & 1,2 \\
2 & 1,00 & 1,00 & 24,9 & 1,0 \\
3 & 1,00 & 1,00 & 1,7 & 1,5 \\
4 & 0,93 & 1,00 & 3,0 & 1,5 \\
5 & 0,96 & 0,94 & 34,3 & 2,1 \\
6 & 1,00 & 1,00 & 24,3 & 10,1 \\
7 & 0,47 & 0,90 & 0,3 & 2,2 \\
8 & 0,94 & 0,88 & 13,6 & 11,8 \\
9 & 0,15 & 0,78 & 4,4 & 3,5 \\
10 & 0,98 & 0,92 & 57,5 & 4,5 \\
11 & 0,88 & 0,97 & 15,2 & 2,2 \\
12 & 0,71 & 1,00 & 28,2 & 7,4 \\
13 & 0,89 & 0,98 & 3,8 & 3,2 \\
14 & 1,00 & 1,00 & 5,1 & 6,4 \\
15 & 1,00 & 1,00 & 5,7 & 1,4 \\
16 & 1,00 & 0,98 & 20,1 & 11,8 \\
17 & 0,81 & 0,57 & 2,5 & 1,5 \\
\hline
\end{tabular}

Table 7 GDR and FDH per patient for SVM and PS-SVM. Each number is the median value of 10 Monte-Carlo simulations. Statistically significant differences $(p<0.05$, Wilcoxon signed rank) between PS-SVM and SVM are highlighted in green (performance increase) or red (performance decrease). Patient numbers highlighted in green correspond to patients where PS-SVM outperforms SVM based on both GDR and FDH or either one of them when the other remains the same.

Classification performance quantified by the two parameters GDR and FDH facilitates SVM versus PS-SVM comparison. However, they may often be not adequate or insufficient when EEG patterns are more complex. As a typical example, figure 23 shows the classifier score of patient 17 who was in status epilepticus during most of the registration. It shows SVM and PS-SVM classifier output scores of an approximately 48 hour EEG recording. This registration shows an episode with gradual PED activity increase (orange box) that eventually developed into episodes with seizures (green boxes). SVM (figure 23A) resulted in a larger number of false detections in the hours preceding the first seizure (classifier score $>0.5$ ). In the same time span, PS-SVM (figure 23B) detected almost nothing because after the first detection the classifier has learned to classify this PED pattern as non-seizure. Simultaneously this resulted in lower classifier scores during the first 


\section{Chapter 5}

seizure episode and it fails to detect any seizure in the second episode. All remaining seizure episodes are, on the other hand, more clearly detected by PS-SVM. Finally, after the last seizure episode, an episode with PED activity caused PS-SVM to produce more false detections. This because this PED activity was similar to the seizures during the last episode and the classifier was constantly adapted with the most recent detections. So, in this registration PS-SVM initially performed better (less false detections) and thereupon worse (missed seizure), then better (seizures more clearly detected) and again worse (more false detections).
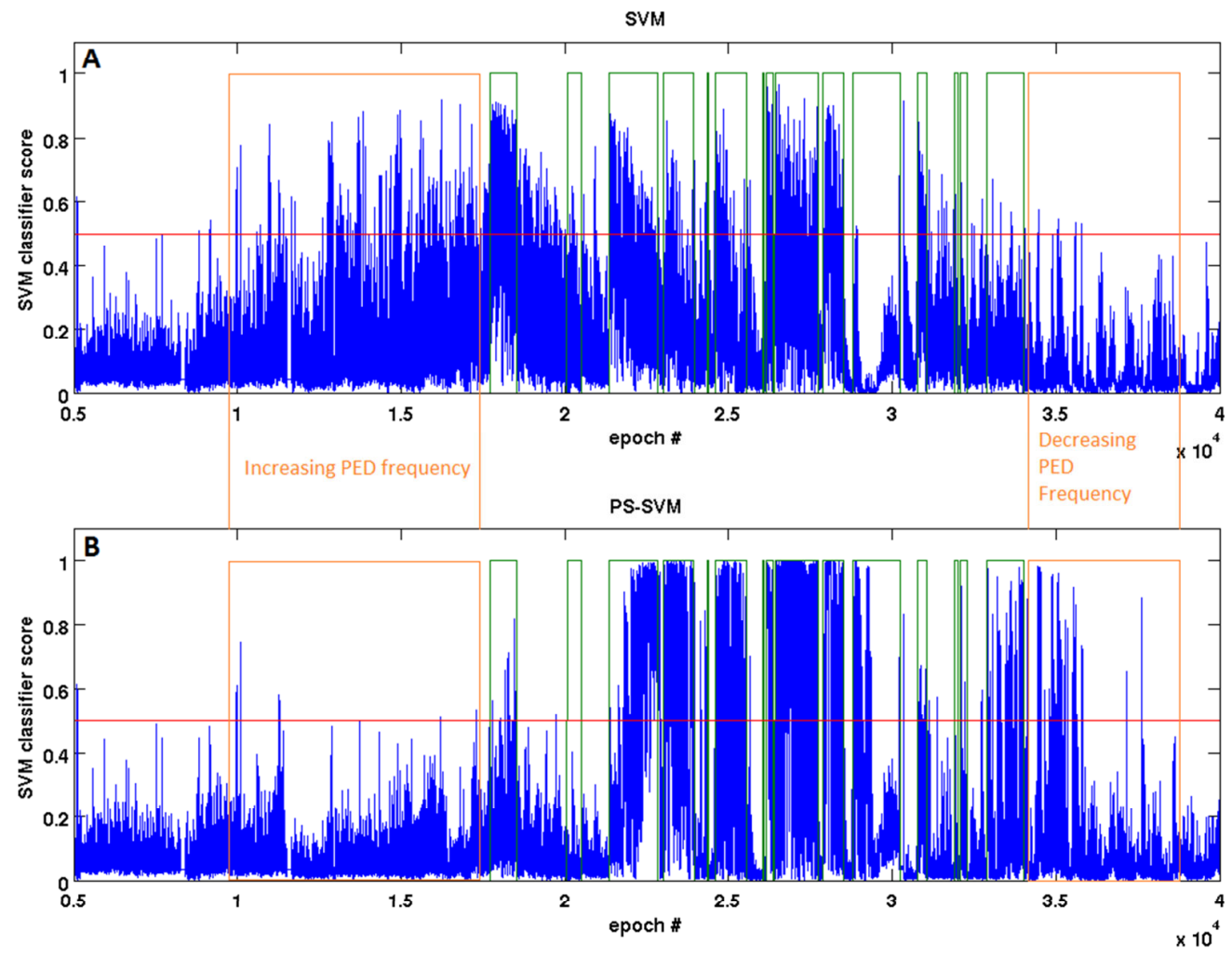

Figure 23 Example of classifier output (epochs $0.5^{*} 10^{4}-4 * 10^{4}$, approximately 48 hours): SVM (top) and PS-SVM for patient 17. An episode with seizures (EEG expert annotations in green) is preceded by an episode of increasing PED frequency and is followed by an episode of decreasing PED frequency. The detection threshold (0.5) is indicated by the red horizontal line. 
Patient specific seizure detection

\section{Discussion}

General discussion

The scientific literature [8-10] describes various methods to improve classifier performance in case of a single patient by using patient specific EEG data. These methods use patient specific EEG data either available beforehand or selected from the test data itself. However, in clinical practice usually no prior patient-specific EEG is available and it is unknown whether or when a patient will have seizures. Furthermore, patient specific data only becomes available when a detection algorithm detects a seizure. Our study showed that with regard to a more patient specific SVM classifier, its performance can be increased by taking into account good and false detections. Practically, EEG epochs that trigged an alarm were added to the original training dataset after which a new classifier was trained. Both false and true detections were used to tailor the classifier to specific EEG characteristics of a patient. In this way, the classifier was better able to detect future seizures and to recognise patient specific EEG patterns as non-seizure that previously would have triggered false alarms. For most patients, PS-SVM resulted in improved performance (10/17 patients). Our study also illustrated that in particular in the presence of periodic epileptiform discharges (PED), PSSVM may show lower performance (2/17). The effect on FDH and GDR of incorporating both seizure and PED epochs into the classifier was sometimes unpredictable. This is because it depends on the particular time-course of seizure and PED events, as illustrated with patient 17. However, PS-SVM performance may depend on whether PED activity precedes or occurs after a seizure (or episode of seizures) and also the particular evolution of PED frequency. In general, an increase in GDR paralleled an increase in FDH and vice versa. These findings underline the fundamental problem that in many cases a classifier cannot distinguish between PED and seizure activity because of their similarity 


\section{Chapter 5}

with respect to the quantitative EEG features used for classification. Not only sophisticated computer algorithms may suffer from this problem but more in particular this holds for human EEG experts who cannot always make a clear distinction between epileptiform and epileptic activity $[23,24]$.

This more fundamental problem should be solved first before a successful attempt can be made to use computerized methods to reliably distinguish PED from seizure epochs. A classifier trained to distinguish PED from seizure EEG patterns can then serve as an extra verification step for epochs classified as seizure. Ultimately, seizure detection should be approached as a multi-class classification task in which epileptiform activity is recognized as an additional class.

Considering the association of PED with clinically overt seizures and clinical outcome [25-27], it is clinically relevant to detect PED as well. Therefore, when interpreting seizure detection performance, false detections due to PED should not be weighted as heavy as false detections caused by other phenomena.

Implications, limitations and future research

This paper focused on challenges met when moving from patientindependent towards patient-dependent seizure detection. Our results showed that significant performance increase can be achieved by incorporating both good and false detections into the SVM classifier. Nevertheless, in particular incorporating false detections due to PED, could result in GDR reduction. In our systematic approach, each detected epoch was used in making the SVM classifier patient-specific. Naturally, during online monitoring a physician can always choose to accept or reject certain epochs. For example, false detections caused by severe PED activity are likely to be rejected by the physician. However, our approach allowed us to identify exactly these cases where adding specific false detection to the SVM classifier leads to lower performance. In a real monitoring setting one could decide after each 102 
detection whether to incorporate this new detection into the patient specific classifier or not. Higher and lower performance, in the context of this paper, should be treated with caution. For example patients 5 and 10 (table 7) showed increased PS-SVM performance for FDH but lowered GDR performance. However, the small and in clinical practice acceptable decrease in GDR, was compensated by a dramatic decrease in $\mathrm{FDH}$. Although PS-SVM versus SVM resulted in reduced GDR, in absolute terms GDR still exceeds $90 \%$ while FDH was reduced to normal values compared to the other patients in our dataset. Another example was patient 7 where both GDR and FDH increased. However, while GDR was almost doubled ( 0.47 to 0.9 ), FDH increased from an extremely low value to a value of 2.2 which was also in the range of values found for the other patients. Therefore, in practise, PS-SVM resulted in better performance for these 3 patients as well.

Another issue was that the SVM classifier often detected not all epochs marked as seizure or did not detect a seizure in all involved channels. After review of the detection by an EEG expert, these missed epochs and channels could also be used for PS-SVM training. It is expected that adding these epochs will result in an even larger performance gain because they were initially not detected and thus add more new information to the classifier compared to epochs already correctly recognised as seizure. However, this required detailed per-channel annotations which were not available to us because of the sheer number of seizures in our dataset. In this study no extra emphasis during SVM classifier training was put on the patient-specific epochs compared to the epochs from the original patient-independent training set. This can be achieved by using a higher penalty factor (boxconstraint) for patient-specific epochs during SVM classifier training [28]. Furthermore, the review of seizure detections by an EEG expert can yield other valuable patient-specific information which can be used to further optimize performance. For example, a subset of features can prove to be more useful for classification for a specific patient. 


\section{Chapter 5}

Furthermore, using information regarding the channels involved in a seizure may further improve performance [29]. To conclude, incorporating both good and false detections into the SVM classifier is an effective way to improve classification performance. However, future research should be focussed on developing best-practise guidelines, especially regarding how to cope with periodic epileptiform activity.

\section{Conclusions}

Our study showed that epileptic seizure detection in comatose patients can significantly be improved by interactively adapting the SVM classifier with patient specific EEG epochs. This was simulated in our retrospective study by incorporating the EEG data of both good and false detections into the original non-patient specific training data set. Subsequent retraining of the SVM classifier on this updated training data set resulted in better classification performance. However, when false alarms are triggered by epileptiform activity such as periodic epileptiform discharges, incorporating patient specific EEG data can result in worse performance. 
Chapter 6

\section{An improved qEEG index for asymmetry detection during the Wada test}

Bogaarts G, Gommer E, Hilkman D, van Kranen-Mastenbroek V, Reulen J. An improved qEEG index for asymmetry detection during the Wada test. Epilepsy \& behavior : E\&B. 2016;62:40-6. 
Chapter 6

\section{Abstract}

The Wada test is commonly used to evaluate language and memory lateralization in candidates for epilepsy surgery. The spatial Brain Symmetry Index (BSI) quantifies inter-hemispheric differences in the EEG. Its application has been shown to be feasible during Wada testing. We developed a method for the quantification of EEG asymmetry that matches visual assessments of the EEG better than BSI. 53 patients' EEG data, with a total of 85 injections was analyzed. In a step-wise, datadriven manner, multiple electrode and frequency band combinations have been evaluated. Eventually, BSI, calculated using only the frontal electrodes $F_{3}$ and $F_{4}$, was combined with a temporal measure of delta power in the central electrodes, $\mathrm{C}_{3}$ and $\mathrm{C}_{4}$, into a new measure: $\mathrm{CBSI}$. Using the area under the ROC curve (AUC), we showed that cBSI performs significantly better relative to BSI (median AUC 0.98 versus $0.96, p=0.0015$, Wilcoxon signed rank test). Our results showed that asymmetry detection was significantly improved by combining temporal with spatial qEEG measures. In the future, our combined qEEG measure could allow for a more objective way of monitoring EEG asymmetry, thereby increasing the feasibility of using EEG as a monitoring tool during the Wada test. Future studies should, however, validate our cBSI method in real time in the operating room or radiology suite. 
Asymmetry detection

\section{Introduction}

The Wada test (77) is commonly used to evaluate cerebral language and memory lateralization in patients being considered for epilepsy surgery. The test consists of the inactivation of one hemisphere by injecting an anesthetic, usually sodium amobarbital (78-80). The anesthetic is injected into one of the internal carotid arteries through a catheter which is inserted percutaneously into the iliac artery $(81,82)$. During the inactivation of one hemisphere, memory and language tests are conducted with the conscious patient to predict the possible risk of amnesia and language loss after temporal-lobe resection.

For a reliable assessment of memory and language lateralization, it is crucial that the initiation, termination, and degree of hemispheric inactivation is known (7). The most common monitoring technique used to assess hemispheric inactivation is visual assessment of ipsilateral delta slowing in the EEG. However, due to low inter-observer agreement of EEG interpretation, this technique is less than optimal (83). Nevertheless, due to the lack of a better alternative, visual assessment of the EEG remains the gold standard (84-86).

Various quantitative EEG analysis techniques (qEEG) have recently been introduced in order to eliminate the subjectivity inherent to visual EEG assessment during the Wada test $(7,87)$. The Brain Symmetry Index (BSI) quantifies inter-hemispheric differences $(7,88)$. It is defined as the absolute value of the relative difference between the spectral density of the right and left hemisphere. Tu et al. showed that the period of hemiplegia corresponded best with changes in delta power, and amplitude-integrated EEG had the highest signal/noise ratio (87). However, BSI is a conceptual measure for global hemispheric asymmetry, and hemiplegia caused by amobarbital perfusion in motor areas may not reflect functioning of deeper mesial structures, which are associated with memory function. Therefore, both BSI, and changes in delta power might not be optimal for a Wada test. 
Chapter 6

Our aim was to investigate whether the combination of two measures, BSI and changes in delta power, might result in better asymmetry detection. To this end, the asymmetry annotations provided by a clinical neurophysiologist were used as the gold standard. During the Wada test, continuous EEG was recorded using 19 electrodes (10-20 system). This allowed us to systematically evaluate each electrode's contribution to the detection of asymmetry. We hypothesized that a combination of $\mathrm{BSI}$ and changes in delta power would result in asymmetry detection that better corresponds to visual assessment.

\section{Methods}

\section{Subjects and Wada tests}

Wada tests of 58 adult patients performed between 2001 and 2012 at Maastricht University Medical Centre were included. Five patient's registrations were rejected due to poor quality of the EEG recording. Patients' age ranged from 19 to 61 (37 \pm 12 , mean \pm standard deviation). Eleven of the remaining 53 patients were injected with 125 mg amobarbital and 42 patients with $150 \mathrm{mg}$. In total the 53 patients received 85 injections: 22 patients received 1 injection, 30 patients 2 and one patient 3 injections. After the amobarbital injection, a clinical neuropsychologist applied a series of memory and language tasks while a clinical neurophysiologist monitored and interpreted the EEG. Memory and language tasks were stopped upon indication of the neurophysiologist that the EEG was symmetric again. All events were recorded and annotated in the EEG (VVK). 
Asymmetry detection

\section{EEG acquisition}

$\mathrm{Ag} / \mathrm{AgCl} \mathrm{EEG}$ electrodes were placed according to the international 1020 electrode placement system, yielding 8 bilateral electrode pairs (Fp1Fp2, F3-F4, C3-C4, P3-P4, O1-O2, F7-F8, T3-T4, T5-T6) and 3 midline electrodes ( $\mathrm{Fz}, \mathrm{Cz}, \mathrm{Pz})$. EEGs were recorded at a sampling rate of $256 \mathrm{~Hz}$ using EEG amplifiers (Schwarzer, type EEG 29 US) and were reviewed online with Brainlab software (r) (OSG).

\section{Calculation of the spatial Brain Symmetry Index}

EEGs were processed off-line with in-house developed Matlab software [14]. The recordings were band-pass filtered between 0.5 and $25 \mathrm{~Hz}$ and segmented into 10 second epochs. Subsequent calculation of qEEG measures was performed on uni-polar EEG channels in the common average derivation. BSI is defined as the absolute value of the average relative differences in spectral density between the left and right hemispheres. Our implementation differs slightly from those published previously $(43,88,89)$. BSI is calculated according to:

$$
B S I=\frac{1}{11 * 8} \sum_{f=1}^{11} \sum_{e=1}^{8}\left|\frac{L_{f e}-R_{f e}}{L_{f e}+R_{f e}}\right|
$$

where $e$ and $f$ indicate the electrode pair, and frequency bin respectively. $L$ and $R$ indicate the power for a left and right electrode respectively. First, the spectral density of eleven $2 \mathrm{~Hz}$ bins ranging from 0.5 to $16 \mathrm{~Hz}$ ( $1 \mathrm{~Hz}$ overlap) was calculated for each EEG channel. Then, for each of the 11 frequency bins, the absolute difference between $L$ and $R$ divided by $L+R$ was calculated for each electrode pair. Next, these values were averaged, first over the 8 electrode pairs and subsequently over the 11 frequency bins. BSI can be calculated using any combination of minimally 1 , and maximally 8 , left-right electrode pairs. With 8 leftright electrode pairs this yields a total of 255 combinations. To 
Chapter 6

investigate the importance of a particular electrode pair, all possible electrode subsets including or excluding this particular pair are compared.

\section{Calculation of a temporal delta power qEEG measure}

First, several qEEG measures containing information about the delta frequency range were evaluated. As a result, log scaled mel-spaced filter bank energy ( $\mathrm{FBE}$ ) in the delta frequency range $(0.7-2.3 \mathrm{~Hz})$ was chosen $(35,66)$. FBE is calculated from a single channel EEG epoch using the following steps:

1. Calculate the Fourier spectrum using the Fast Fourier transform

2. Calculate the mel-spaced filter bank containing 15 triangular filters

3. Apply the mel-spaced filter bank to the power spectra

4. Sum the energy in the second filter (corresponding to $0.7-2.3 \mathrm{~Hz}$ )

5. Log scale the summed energy

Subsequently, relative FBE ( $\mathrm{FBBE}$ ) was calculated expressing actual FBE relative to its median FBE value of the 5 minutes before injection (87). Also for the midline electrodes ( $F z, C z$ and $F z), r F B E$ was calculated to obtain a complete overview of all electrodes without excluding potential useful information beforehand. rFBE is calculated for each of the 19 electrodes. However, depending on the injection side, either the electrodes from the left or right hemisphere are of interest. Therefore, to obtain one measure for each left-right electrode pair, independent of the injection side, the maximum rFBE value of each left-right electrode pair is taken:

$$
r F B E_{L-R}=\operatorname{Max}\left(r F B E_{L}, r F B E_{R}\right)
$$


where Le and Re indicate a left and right electrode, for example $F_{3}$ and $F_{4}$. This yields a total of $11 \mathrm{rFBE}$ values including the 3 midline electrodes.

\section{Combining BSI and rFBE into one combined asymmetry score}

The goal of qEEG as a monitoring tool is assisting a clinical neurophysiologist with his/her visual evaluation of the EEG, in particular to classify EEG epochs as symmetric or asymmetric. To this end, BSI and rFBE have to be combined into a single score (cBSI). To accomplish this, Linear Discriminant Analysis (LDA) is used. LDA has been proven to be useful for asymmetry detection of post-clamp EEG changes during carotid endarterectomy (8). LDA is a method which optimally separates two classes by finding a linear combination of a set of features (37). In this study we use LDA to find a linear combination of BSI and rFBE best separating symmetric from asymmetric EEG epochs. To avoid overfitting and subsequent over optimistic classification results, the patient based leave-one-out (LOO) cross validation scheme is used. With LOO, an LDA model is trained on all but one patient's data. Thereupon this model is used to calculate cBSI for the left out patient's data which serves as the test data. This procedure is then repeated for all patients. Before LDA model training, BSI and rFBE in the training data were normalized by subtracting their mean $(\mu)$ and dividing by the standard deviation $(\sigma)$ to make them commensurable. Normalization of the test data was performed with the means and standard deviations of the training data:

$$
\mathrm{cBSI}=\mathrm{a} * \frac{\mathrm{BSI}-\mu_{\mathrm{BSI}}}{\sigma_{\mathrm{BSI}}}+\mathrm{b} * \frac{\mathrm{rFBE}-\mu_{\mathrm{rFBE}}}{\sigma_{\mathrm{rFBE}}}
$$

Parameters $a$ and $b$ in equation 14 are weight factors to be optimized by LDA. Finally a Kalman filter (KF) (90) is used to smooth the BSI, rFBE, and $\mathrm{CBSI}$ time series. A KF is an algorithm that uses a series of noisy 


\section{Chapter 6}

measurements (asymmetry scores) to produce estimates of an underlying system (actual hemispheric inactivation), state being more precise then when based on single asymmetry measurements alone. A $\mathrm{KF}$ is especially useful for online tasks such as asymmetry detection during a Wada test because it does not introduce a computational delay in contrary to for example a central moving average filter (7).

\section{Performance evaluation}

Performance for BSI, rFBE and cBSI was evaluated for the ensemble of epochs from all patients, as well as per patient separately. Every 10 second EEG epoch was considered an individual observation for which $\mathrm{BSI}, \mathrm{rFBE}$ and $\mathrm{CBSI}$ were calculated. By applying a detection threshold to a $q E E G$ scores, each epoch was classified as either 'symmetric' or 'asymmetric'. Based on the annotations provided by the neurophysiologist, sensitivity and specificity were calculated. Sensitivity and specificity were calculated for the complete dataset and for each patient separately.

To evaluate classification performance, an ROC curve is obtained by plotting sensitivity versus 1-specificity for all possible detection thresholds. The area under this curve (AUC) is used as a performance measure. AUC ranges between 0.5 for random and 1 for perfect classification. Classification performance was evaluated by means of two different AUC values; method 1: calculated on all epochs in the dataset, and method 2: calculated per patient individually. The first method yields a single AUC value per asymmetry detection method (BSI, rFBE and CBSI) whereas the second yields 53 AUC values. Classification performance differences between asymmetry detection methods were tested for statistical significance using the nonparametric Wilcoxon signed-rank test. To evaluate BSI, classification performance with regard to inclusion and exclusion of a particular electrode pair, the non-parametric Wilcoxon ranked-sum test is used. Pvalues below 0.05 were considered statistically significant. 
Asymmetry detection

\section{Results}

\section{BSI electrode subset evaluation}

Figure 24 shows AUC value distributions for BSI for all possible electrode pair combinations divided into two groups. Applying method 1, the AUC values were calculated using all epochs in the dataset. The left box plot is the group where a particular electrode pair (annotated at the top) was excluded and the right box plot where this electrode pair was included to calculate BSI. The horizontal blue lines indicate the AUC value for the single annotated electrode pair. When F3-F4 is included in a subset of other electrode pairs, AUC values are significantly higher compared to electrode subsets without F3-F4 (median AUC with F3-F4: 0.89, median AUC without F3-F4: 0.83, $\mathrm{p}<0.0001$, Wilcoxon ranked sum test). Only 3 other electrode pair combinations, including F3-F4, yield a higher performance (F3-F4 + P3-P4, F3-F4 + C3-C4, and F3-F4 + C3-C4 + O1-O2) compared to F3-F4 alone (method 1). However, when the AUC values were calculated per patient (method 2), the Wilcoxon signed rank test indicates that the differences in AUC values between F3-F4 and the 3 aforementioned subsets are not statistically significant ( $p=$ $0.52, p=0.57$ and $p=0.85$ ). Therefore, BSI based on the single electrode pair F3-F4 is optimal and subsequently used for the calculation of $\mathrm{CBSI}$.

Single electrode pair evaluation

To investigate the importance of individual electrode pairs for BSI based asymmetry detection, AUC values for BSI calculated on single electrode pairs were calculated per patient. Figure 25A shows the AUC values for the BSI calculated on single left-right electrode pairs. Each boxplot indicates the distribution of the AUC values for the 53 patients. BSI based on electrode pair F3-F4 results in the highest median AUC value 
Chapter 6

(0.96) which is significantly higher compared to all other single electrode pairs ( $P<1 \mathrm{e}-6$, Wilcoxon signed rank).

rFBE frequency and electrode evaluation

Figure 25B shows rFBE AUC value distributions for each electrode pair including the 3 midline electrodes. The best performance (median AUC $=0.96)$, is achieved by $\mathrm{Cz}$ and C3-C4 ( $p=0.42$, Wilcoxon signed rank test). Also P3-P4 shows good performance (median AUC $=0.95$ ) but slightly less compared to $\mathrm{Cz}$ ( $p=0.047$, Wilcoxon signed rank test). In contrast to BSI which performs best for the frontal electrodes, rFBE performs best for the central, posterior and temporal regions.
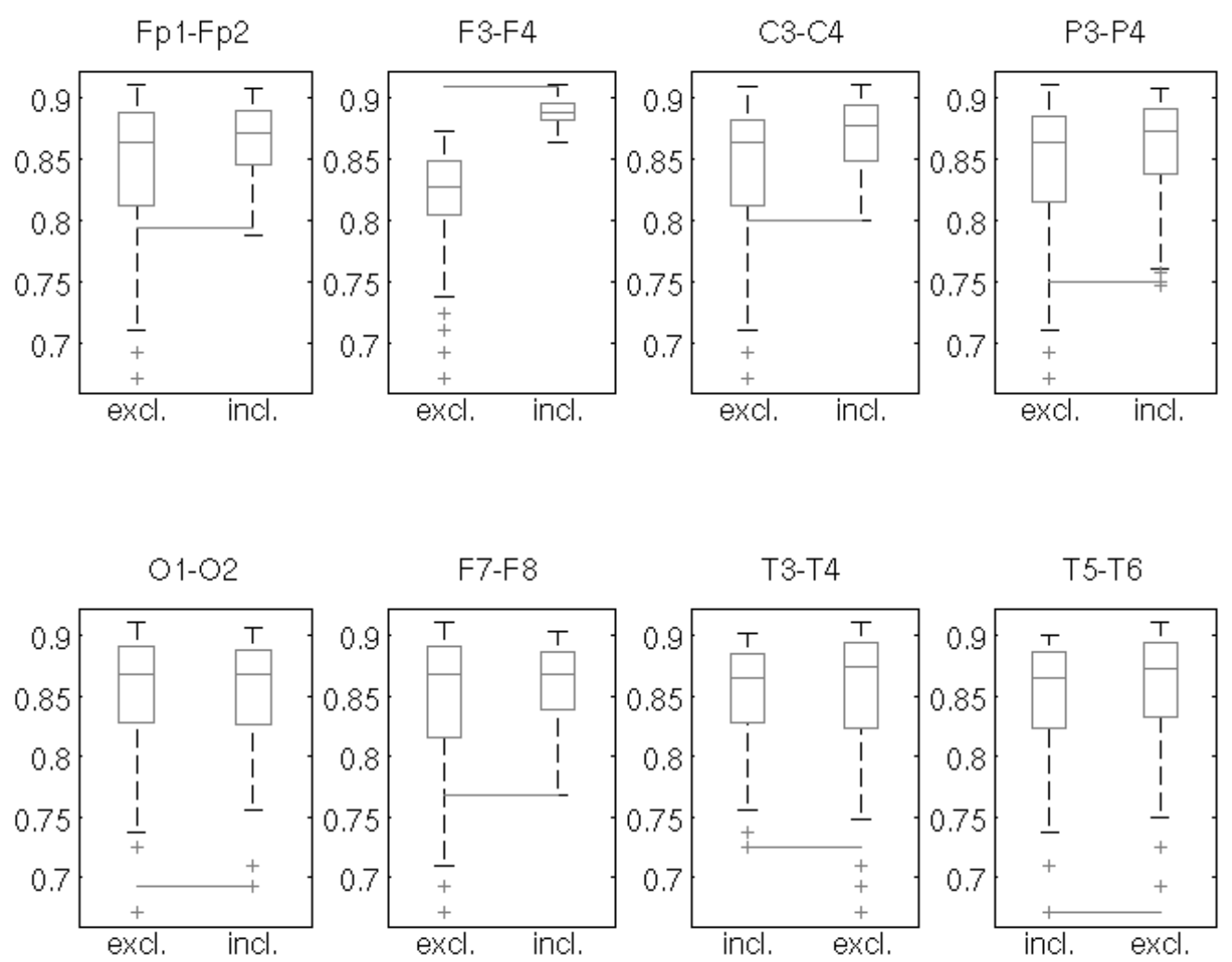

Figure 24 BSI AUC value distributions for the complete dataset (method 1), calculated for all possible electrode pair combinations excluding (127 electrode subsets, left box plot) or including (128 electrode subsets, right box plot) a particular electrode pair. The blue line indicates the AUC value for the BSI based on the single electrode pair 

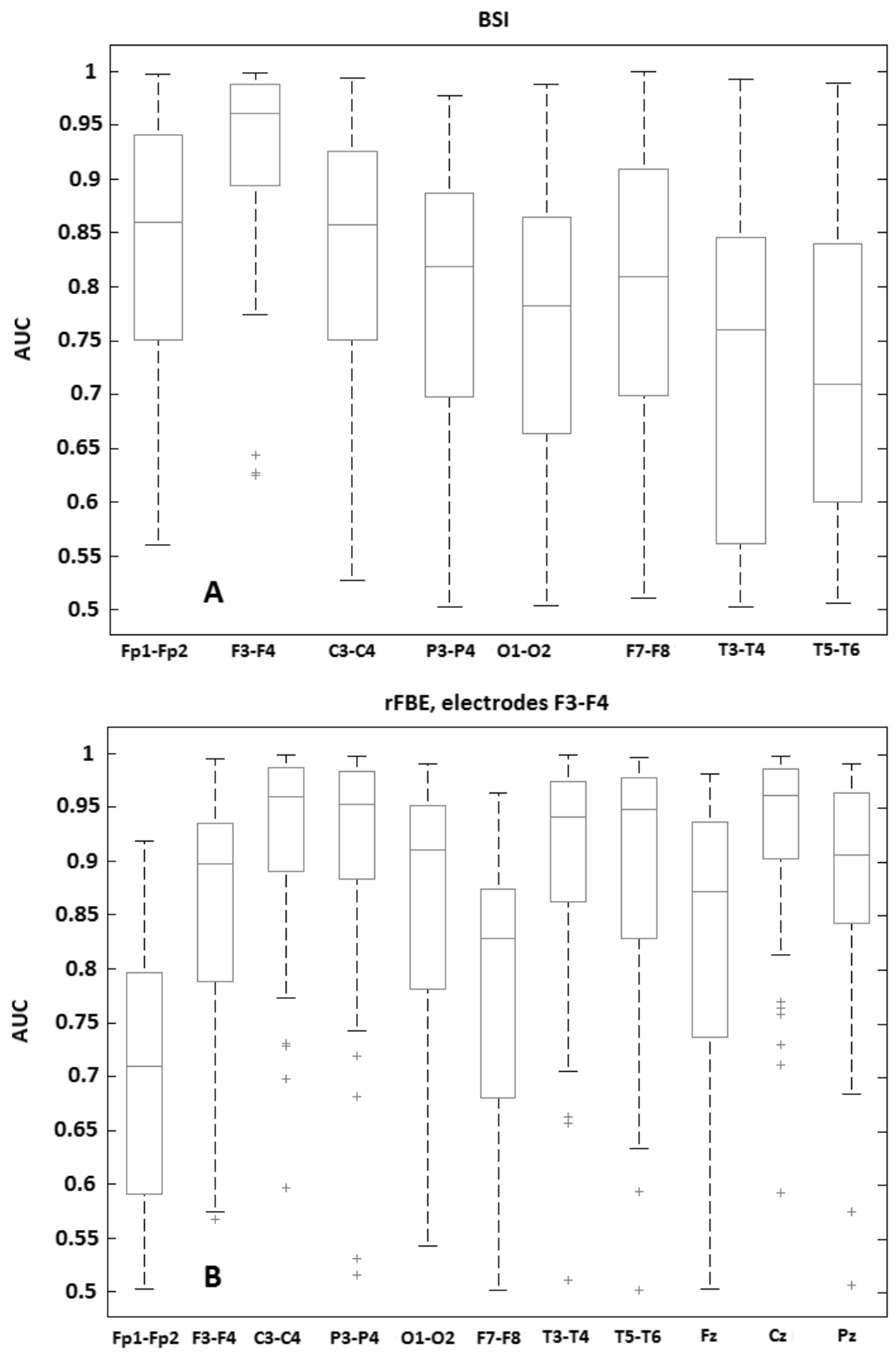

Figure 25 AUC value distributions for BSI for each electrode pair (A). AUC value distributions for $r F B E$ for each electrode pair and midline electrodes (B). Each box plot consists of the AUC values calculated for each of the 53 patients separately (method 2). 
Chapter 6

Time course of BSI per frequency

Figure 26A shows the BSI response averaged over 85 injections split up per frequency band for electrode pair F3-F4. To reveal the average shape of the BSI response, visually marked 'asymmetric' periods were scaled in time by interpolation before averaging. For the lowest frequency band, the BSI response has a delayed onset and returns to baseline at roughly $2 / 3$ of the total asymmetry period. BSI based on the power in the $2-4 \mathrm{~Hz}$ band has the highest average response but also returns to baseline before the end of the asymmetry period. BSI based on lower frequencies alone would therefore underestimate the duration of asymmetry. A remarkable observation is that the BSI response of the two higher frequency bands continues to rise when the other frequency BSI scores start to decline. As described in the methods section, the BSI score is calculated on all frequency bands from 0.5 to 12 $\mathrm{Hz}$. In this way, the contribution of the higher frequencies in the BSI calculation compensates for the too early decline of the lower frequencies. This is also apparent from Figure 26B which shows that BSI scores calculated on all frequency bands performed significantly better compared to separate frequency bands $(p<1 e-5$, Wilcoxon signed rank test). 


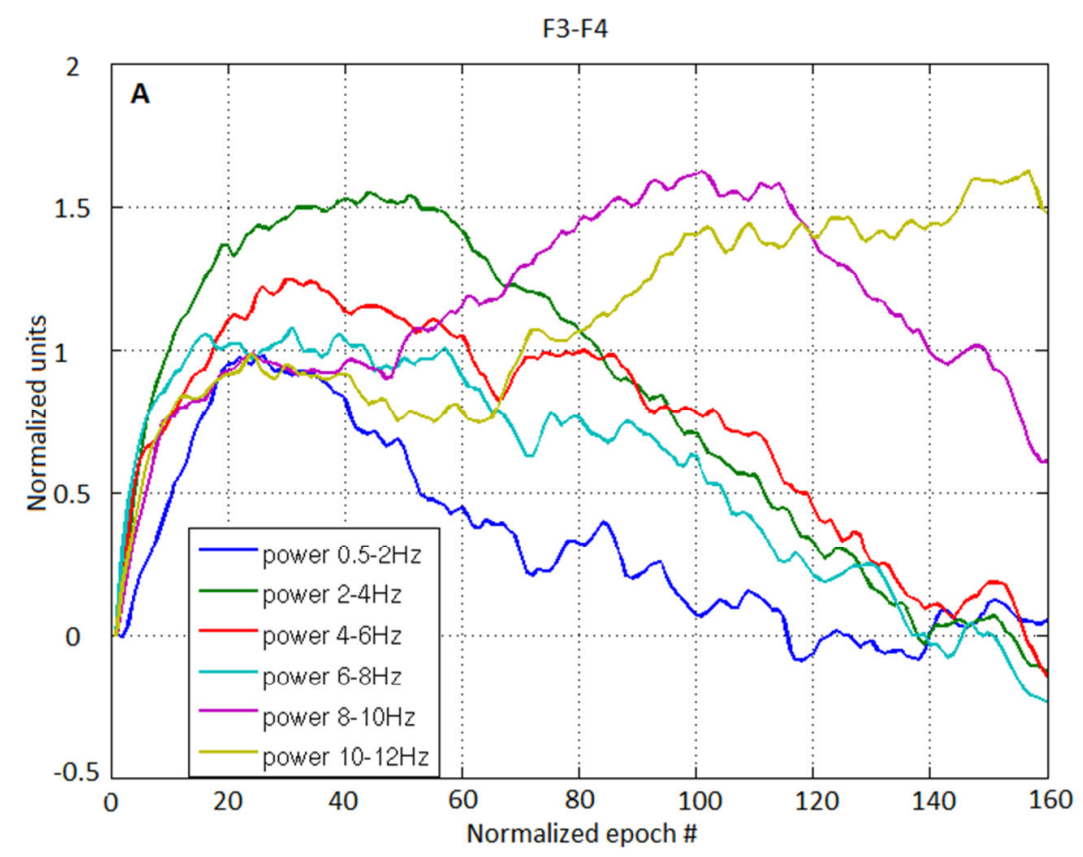

BSI, F3-F4

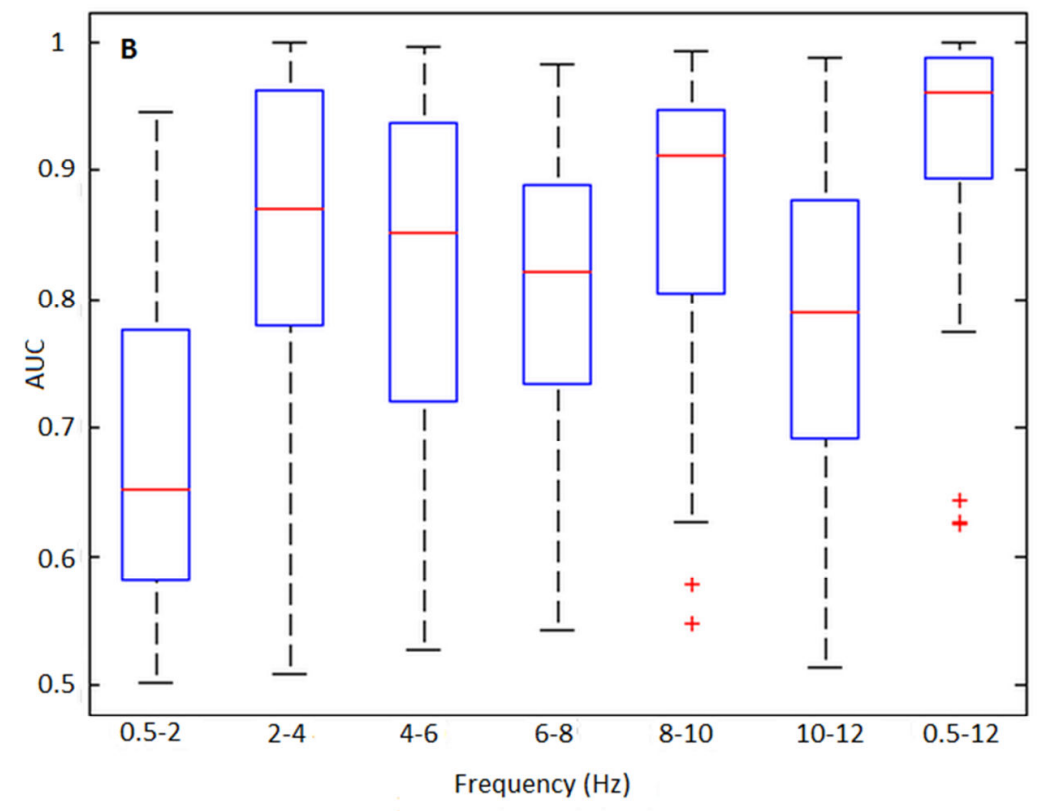

Figure 26 Averaged BSI response after amobarbital injection for the entire period marked as 'asymmetric'(A). AUC value distributions for BSI calculatedfor separate frequency bands (boxplot 1-6) as well as all frequency bands $(0.5-12 \mathrm{~Hz}$, boxplot 7) (B). 
Chapter 6

\section{LDA combination of BSI and rFBE}

To construct a final cBSI score, BSI calculated using electrode pair F3-F4, and $\mathrm{rFBE}$ based on electrode pair $\mathrm{C} 3-\mathrm{C} 4$, were combined using LDA. Figure 27 shows a scatter plot of all rFBE versus BSI points: red points mark 'symmetric' and green points 'asymmetric' epochs. The orientation of the green versus red points indicate that a (linear) combination of rFBE and BSI better separates 'symmetric' from 'asymmetric' epochs compared to either rFBE or BSI alone. In each LOO evaluation, an LDA model was trained on a different subset of patients, consequently different sets of LDA weights were found (BSI: $0.67 \pm 0.01$ and rFBE $0.74 \pm 0.01$ ). Because the weights for rFBE and BSI were of similar magnitude, and optimized for the LOO training dataset and not for the left out patient, we simplified the linear combination of BSI and rFBE by taking the average of both (normalized) measures which equates to setting equal LDA weights for BSI and rFBE. This simplification did not result in statistically significant different results ( $p$ $=0.5$, Wilcoxon signed-rank test).

The blue line in figure 27 indicates the direction perpendicular to the linear combination of rFBE and BSI. Depending on the desired sensitivity and specificity, a line perpendicular to the line in figure 27 can be used for classification. By varying this line, the ROC curve in Figure 28 is constructed. 


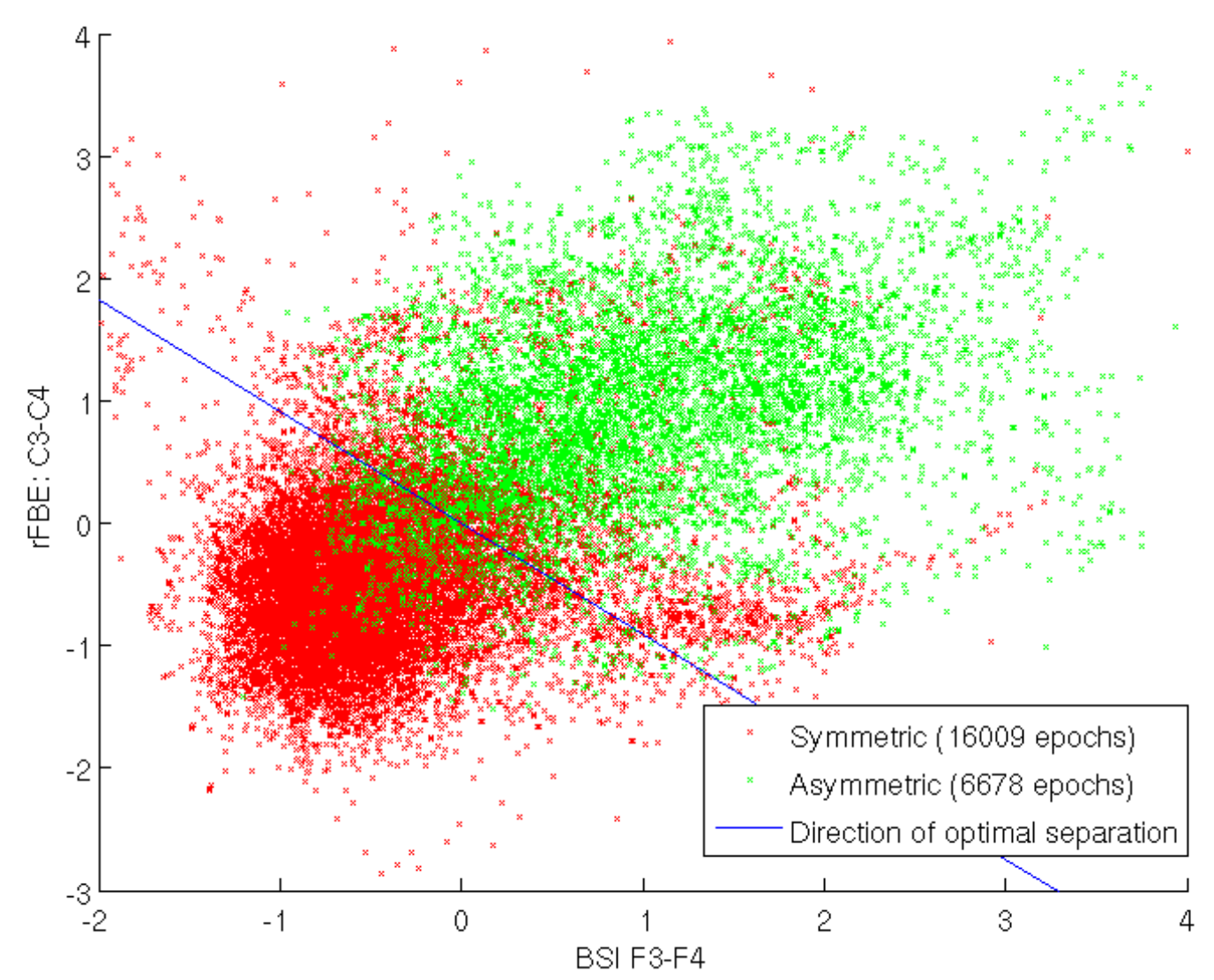

Figure 27 Scatterplot of BSI-rFBE data points. BSI and rFBE values are normalized to a z-score. Red points illustrates epochs labeled as 'symmetric' and green points epochs labeled as 'asymmetric'. Optimal classification can be achieved by lines parallel to the blue line.

\section{Classification performance}

Figure 28 summarizes classification performance for BSI, rFBE and cBSI. Figure 28A shows the ROC curves for the complete dataset (method 1). In figure 28B, AUC values are calculated for each of the 53 patient separately and their distributions displayed as boxplots (method 2). Both the higher area under the ROC curve and the per-patient AUC values show that a significant improvement in classification can be accomplished by combining BSI and rFBE. This improvement is reflected 


\section{Chapter 6}

in the AUC values calculated for the entire dataset (from 0.91 to 0.95 ) as well as in the AUC values calculated per patient (from 0.96 to $0.98, p$ $=0.0015$, Wilcoxon signed rank test). Furthermore, the increase in median AUC value corresponds to a relative increase of 50\% (100* 0.98 $0.96) /(1-0.96)$, which is of similar magnitude to the $45 \%$ increase in AUC calculated for the entire dataset.
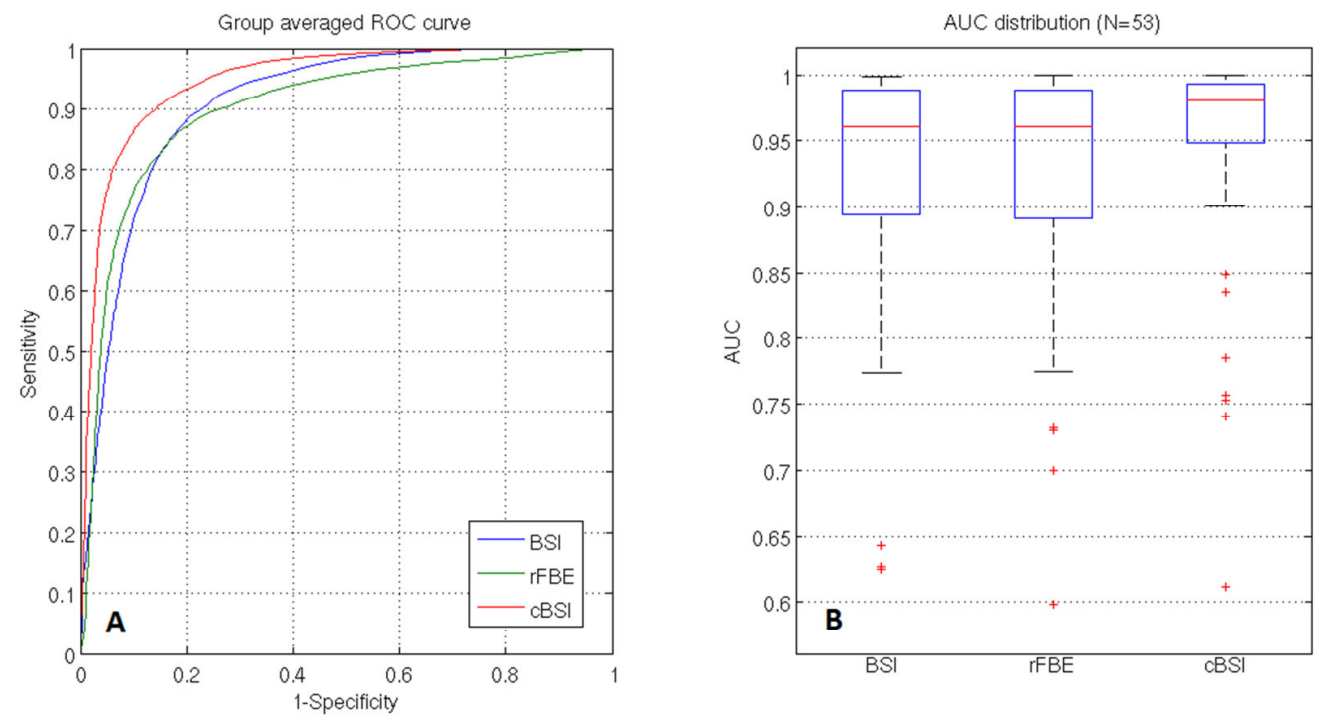

Figure 28 ROC curves calculated for the entire dataset (method 1)(A). AUC distributions for BSI, rFBE and CBSI (B). Each box plot illustrates the AUC distribution of the 53 patients (method 2).

\section{Discussion}

\section{Asymmetry detection Performance}

In this paper, we used a data-driven approach to improve EEG asymmetry detection during the Wada test. Our optimization was based on two previously defined qEEG measures: the Brain Symmetry Index (BSI) (7) and the relative Filter bank Bank Energy (rFBE) (87). Furthermore, we evaluated which electrodes resulted in optimal asymmetry detection performance when used to calculate BSI and rFBE . 
Electrode pair F3-F4 resulted in the highest performance for BSI and either electrode pair $\mathrm{C} 3-\mathrm{C} 4$ or the mid-line electrode $\mathrm{Cz}$ resulted in the highest performance for $r F B E$. Taking the average of both qEEG measures resulted in an $A U C$ increase from 0.96 (BSI, rFBE) to 0.98 (CBSI), corresponding to a relative increases in $A U C$ of $50 \%$. It can therefore be concluded that delta slowing in the central and frontal region corresponds best with the clinical neurophysiologist's visual EEG assessment. However, this contradicts the clinical neurophysiologist's assertion that ipsilateral slowing (delta) in temporal regions should be the focus. However, as shown in figure 26, a decrease in asymmetry for lower frequencies is compensated for by an increase in asymmetry in the higher frequency range $(8-12 \mathrm{~Hz})$, resulting in better agreement with visual assessment. The latter might therefore serve as an extra hint for the clinical neurophysiologist that hemispheric inactivation is about to end.

The goal of asymmetry detection during the Wada test is to make sure that memory and language tests are only conducted when the injected hemisphere is deactivated. Therefore it is most important that the algorithm does not classify symmetric epochs as asymmetric, hence specificity is of higher importance then sensitivity. This implies that only a very high specificity (e.g. 95-99\%) is acceptable which would, considering the characteristic shape of the cBSI response, results in shorter asymmetry periods and hence less time to conduct memory and language tests. For example $95 \%$ specificity would result on average in $80 \%$ sensitivity which equates to $20 \%$ less time to conduct memory and language tests.

\section{Directions for future research}

We took a data-driven approach to show the importance of the central and frontal regions of the brain for qEEG-based asymmetry detection 


\section{Chapter 6}

during the Wada test. Another approach one could take is to make a priori assumptions about EEG channel importance for asymmetry detection. The Wada test evaluates language and memory lateralization, where language is mainly located in the inferior frontal gyrus (Broca's area) and superior temporal gyrus (Wernicke's area), and memory in the temporal lobe. Therefore, it could be hypothesized that temporal electrodes are likely to be of higher importance. Because of the anatomy of the vasculature in the brain, intra-carotid amobarbital would likely affect the central areas of the brain, such as the motor strip, more than it would the mesial structures, such as the hippocampus (23). In fact, the duration of slow waves in the mesial temporal region, corresponding to memory function, are significantly shorter than in the central region $(23,91)$. Furthermore, the presence of significant, but less evident, contra-lateral qEEG changes, even in the absence of significant cross-filling (observed by angiography), suggests that contralateral EEG changes may not only be induced by direct drug perfusion into the contralateral hemisphere, but also by a transient functional disconnection from the inactivated ipsilateral hemisphere (87, 92-95). Contralateral EEG changes due to cross-filling occur mainly in the anterior cerebral artery region (87), and the occipital electrodes could be less relevant for this reason. It is also common to exclude the mid-line electrodes ( $\mathrm{Fz}, \mathrm{Cz}$ and $\mathrm{Pz}$ ) from the qEEG analysis because they are regarded to be irrelevant for quantifying inter-hemispheric differences (95). However, our results showed that they actually are relevant for asymmetry detection.

The combination of qEEG data, human scoring of the EEG, and a priori knowledge about the structure and functioning of the brain can drive the development of an optimal qEEG measure. Furthermore, it provides the clinical neurophysiologist with additional objective information. This information could guide the clinical neurophysiologist to make more objective decisions. For example when an algorithm indicates that 
asymmetry in the lower frequencies in the temporal regions differs from visual assessment, this information can be taken into account.

Limitations

In this study, EEG data of 53 patients was used to optimize EEG asymmetry detection during the Wada test. LDA weights where optimized using LOO cross-validation. This means that for each patient, the LDA weights where optimal for the other 52 patients. After observing that the weights, optimized in each LOO fold, where very similar we concluded that defining $\mathrm{CBSI}$ as the average of BSI and rFBE yielded the same results as the LOO LDA approach. Strictly speaking, the selection of optimal features should also be performed with a separate train and test set, e.g. LOO cross-validation. However, considering the magnitude of the differences in performance between frequencies (Figure 26B), and between electrodes (Figure $24 \& 25$ ), leaving one patient out would not result in a different set of optimal features. In case of a classification method such as Support Vector Machines and using many different features, the degrees of freedom and consequently the risk of overfitting is substantial and using a separate train and test set is essential.

High quality EEG recordings are essential for the accurate detection of asymmetry using qEEG. Several mathematical methods may be useful to remove artifacts in the EEG before BSI and rFBE are calculated (27-30). In this study, we used a Kalman filter to smooth the BSI and rFBE time series. In this way, short-term artifacts that affected the qEEG measures could be removed. Coincidentally, the difference in performance between asymmetry detection performed with Kalman filtering and asymmetry detection performed without Kalman filtering is of similar magnitude to that found in EEG-based seizure detection (16).

In recent years, several non-invasive alternatives for the Wada test have been investigated of which $\mathrm{fMRI}$ is the most promising (31). $\mathrm{fMRI}$ is a reliable tool for language lateralization in case of strong leftlateralization (32). Therefore, fMRI should be considered for initial 
preoperative assessment of hemispheric language dominance (33). However, the Wada test is still warranted when $\mathrm{fMRI}$ fails to show clear left-lateralization (32). Furthermore, fMRI is an activation procedure which demonstrates areas that participate in a task but does not necessarily demonstrate all areas necessary for accomplishing that task (34). Lateralization of memory function using fMRI is still problematic (35), another important reason the Wada test is still routinely used. Transcranial magnetic stimulation (TMS) is another method that has been suggested a potential alternative to the Wada test (36). When applied to the scalp, the magnetic field induces electrical activity in the underlying brain tissue, temporarily disrupting local cortical information processing. In this way, a brain region can be temporarily deactivated, in contrast to the Wada test where a complete hemisphere is deactivated. Furthermore, TMS does not suffer from the drawbacks of activation procedures such as FMRI, NIRS, fTCD, SPECT and PET (31).

Another commonly used technique to asses hemispheric inactivation is a series of contralateral hand-strength tests (1). The start and end of a successful Wada test are respectively based on the moments at which contralateral hemiplegia occurs and contralateral hemiparesis begins to recover (1, 37-39). However, this technique has several limitations. To test arm strength, the patient has to cooperate, which may distract the patient from memory and language tests (5). Furthermore, there is no standardized procedure for the execution and frequency of the handstrength test. An additional drawback of this test is that it does not provide continuous data and tends to overestimate the duration of hemispheric inactivation (40).

Another limitation is that only one clinical neurophysiologist provided annotations. Our findings suggest that the clinical neurophysiologist may be intuitively taking other cues from the EEG for symmetry detection than presumed. It would therefore be interesting to use 
annotations of multiple clinical neurophysiologists to evaluate interobserver differences in detail.

\section{Conclusions}

Our newly introduced cBSI method offers a significant improvement for the automated detection of asymmetry in the EEG during the Wada test. This improvement lies in the combination of left-right hemispherical differences ( $\mathrm{BSI}, 0.5-12 \mathrm{~Hz}$ ) with temporal changes in the delta frequency range. As a result, cBSI better agrees with visual assessment of EEG asymmetry. Furthermore, we provide insight into dynamic changes in the EEG during the Wada test by evaluating the time course of $\mathrm{BSI}$ and $\mathrm{rFBE}$ response per EEG electrode and per frequency band. When provided in real-time, this insight could assist the clinical neurophysiologist during the Wada test. Visual assessment together with anatomical and functional knowledge of the brain, could guide the development of an optimal qEEG measure for asymmetry detection during the Wada test. 

Chapter 7

General discussion 


\section{Chapter 7}

In comatose patients, excessive metabolic demand and increased blood flow associated with ictal activity may compromise at-risk brain tissue. For this reason it is relevant to detect seizures using quantitative EEG (QEEG). Considering the complicated and time consuming nature of visual EEG inspection, automated and reliable seizure detection in human scalp EEG is of major importance. This is particularly true with regard to acceptance and application of long term EEG monitoring in a clinical environment. As reviewed by Ramgopal (63), scientific research mainly focuses on different EEG classification methods and QEEG features used to detect seizures. In this thesis, improvements in seizure detection performance were described, in particular with regard to classifier training and subsequent testing on EEG data from different patient groups, i.e. neonates and adults. These improvements were achieved by introducing a feature baseline algorithm, evaluation of optimal training data set composition for age-independent seizure detection, automated baseline update, and a way to move from nonpatient to patient specific seizure detection. In addition to seizures, an asymmetric distribution of cortical activity between the two hemispheres might be another sign of inadequate blood supply. The detection of asymmetry is therefore another important part of long term EEG monitoring. Chapter 6 describes how automated asymmetry detection during Wada testing can be improved by combining temporal and spatial QEEG measures. This specific application of asymmetry detection is not directly related to asymmetry detection during long term EEG monitoring. However, due to the clear asymmetry inflicted by the injection of an anesthetic, it is very well suited as a starting point for the development of asymmetry detection methods. In the next sections, specific methodological aspects will be discussed: SVM classifier training, feature normalization, and patient specific seizure detection. Furthermore, clinical applications of automated seizure and asymmetry detection will be discussed. The general discussion will be completed with a critical view on the study limitations and future 
research directions as well as a list of the most important conclusions of this thesis.

\section{Methodological aspects}

\section{SVM classifier training}

Support vector machine (SVM) based seizure detection constitutes current state of the art methodology. SVM can use a large set of EEG features, each of which already may be a classification parameter on its own. It should be emphasized that, given a suitable training/testing method and EEG feature set is used, actual SVM classification performance mainly depends on the quality of the training data. The number of EEG registrations we used for training and testing is mainly limited by the enormous effort it took for an experienced EEG expert to supply detailed annotations to in particular long term EEG registrations. The outcome and quality of an SVM based classifier depends on the quality and reliability of these "golden standard" annotations since SVM outcome is compared to these annotations.

Ideally an EEG training data set, for patient-independent seizure detection, should contain all possible types of seizure and non-seizure EEG patterns, both present in an equal amount. Then each pattern and/or seizure type contributes equally during classifier training and classifier evaluation. We expect that further research into optimal training dataset composition should lead to further gains in. The necessity of an adequate training data set is exemplified by the differences in classification performance between classifiers trained on either neonatal or adult EEG data. It would therefore be very useful to develop an evaluation method to predict whether the addition of a new EEG registration to the training data set will result in a better performing classifier. For example a seizure type not similar to any seizure already in the training data set should be more useful than a 


\section{Chapter 7}

typical seizure already present in the training data. Because this requires a large number of EEGs, a large multi-center collaboration would be the starting point for such an approach. Our research described in chapter 3, optimized age-independent seizure detection by making a distinction between adult and neonatal EEG. However, further training data set optimization can be performed by making other distinctions such as different seizure types, different kinds of epileptiform activity, and other rhythmic non-epileptiform activity such as frontal intermittent delta activity.

\section{Feature normalization}

With regard to classification procedures, we observed significant baseline feature value differences between patients and within patients over time. A typical seizure EEG feature value in one patient can be a typical non-seizure value for another patient and vice versa. As a result, when a training dataset is composed of EEG data from different patients, inter-patient variability will give rise to many contradicting patterns. Consequently, the task of finding a clear separation, for example by training an SVM classifier, becomes unnecessarily complicated because these inter-patient differences could be easily corrected. In chapter 2 we showed that relative differences between seizure and non-seizure EEG properties where similar for each patient. This allowed for simple linear correction for inter-patient feature baseline differences. This so called feature baseline correction (FBC) did not only result in better SVM classification but also reduced classifier training time and the number of support vectors. This indicates that separation of seizure from non-seizure EEG epochs in a dataset consisting of many patients is simplified by FBC. FBC estimates correction factors based on non-seizure EEG baseline feature values. These seizure-free baseline EEG epochs can be manually selected at the start of a registration. Our evaluation of this method on long term EEG registrations, lasting up to several days, revealed that it is not sufficient 
to select a baseline segment only at the start of the registration. Due to dynamic changes in background EEG characteristics, a baseline segment used for FBC may only be valid for a limited time. Due to medication, sleep-wake cycles and changes in the condition of the patient, baseline EEG characteristics may change throughout a registration. It is therefore necessary to dynamically update the non-seizure EEG baseline used for FBC. However, only EEG without artifacts, epileptiform activity, and other patterns that might corrupt FBC should be included in the updated baseline. To this end, we introduced a novelty detection algorithm to select those new EEG epochs suitable for updating the baseline. Our research described in chapter 4 showed that automated baseline update based on novelty detection results in significantly higher seizure detection performance. In particular, it results in less false detections.

\section{Patient specific detection}

FBC is a first step towards patient specific seizure detection. In general, the difficulty of patient independent seizure detection is that one does not know beforehand what the EEG characteristics of a newly monitored patient are. Therefore, it is important to supply the classification algorithm with as much patient specific (EEG) information as possible. This can be done by including EEG epochs into the training data set, either from a previously recorded EEG of this patient or on-line during an ongoing registration. The need for this follows from the fact that, using the approach described in thesis, still not all seizures are detected and false detections still occur. Chapter 5 describes a scenario where the SVM classifier is updated when it classifies an epoch as seizure. It is assumed that upon detection, an EEG expert is notified who subsequently reviews the EEG epoch(s) classified as seizure. Then the expert may decide to add the newly detected epochs to the training dataset used to re-train the existing classifier. In case of a positive detection the epochs are labeled as seizure while false detections are 


\section{Chapter 7}

labeled as non-seizure. A new SVM classifier is then trained on this updated training data set. Further research should reveal which EEG patterns should or should not be included to make the SVM classifier patient specific. For example, our study reveals that epochs with periodic epileptiform discharges (PED), which based on their EEG feature values sometimes closely resemble a seizure, might actually decrease seizure detection performance. By adding these seizure-like epochs as non-seizure, the risk increases that seizure patterns will also be classified as non-seizure. As discussed in chapter 5, in particular PED activity warrants the introduction of a third class in addition to the seizure and non-seizure class. In this way, EEG classification is approached as a multi-class problem. Although not investigated in our study, also other types of patient specific information can be obtained during monitoring. For example, knowledge of in which channels seizures are manifested can be used to improve seizure detection performance $(96,97)$. To conclude, we showed in chapter 5 that updating the SVM classifier with both good and false detections significantly improves seizure detection performance. However, this could also result in worse performance when non-seizure epochs closely resembling seizure activity are included.

\section{Clinical application of automated seizure detection.}

If performed at all, seizure detection during long term ICU monitoring is usually done manually. To reduce the immense workload burden and to make EEG monitoring feasible in daily practice, automated seizure detection may be of great value. A first application would be to use the SVM seizure detection classifier output as a new unique QEEG measure. This measure can then be used, together with other QEEG measures, to provide the clinician with a compact overview of hours of EEG. Based on the information contained in the SVM classifier output, the clinician can decide, retrospectively, which parts of the EEG should be reviewed. In case of many seizure detections, the clinician may review the detections 
both in a chronological order or based on the posterior seizure probability, e.g. with what certainty the EEG is classified as seizure. For this application, it is particularly important not to miss any seizures, whereas false alarms are less important. An SVM classifier with this property can be trained by putting more emphasis on seizure epochs relative to non-seizure epochs. In this way, sensitivity can be increased, however, at the cost of lower specificity (98). The second, more challenging, application of automated seizure detection is to use the automated seizure detection algorithm as a real time alarm system. To this end, a suitable detection threshold for the posterior probability produced by the SVM classifier has to be chosen such that the false detection rate is kept reasonably low to avoid 'alarm fatigue'. Alarm fatigue occurs, especially in an ICU monitoring setting, when so many alarms are triggered that the person responsible for acting upon an alarm, loses confidence in the automated detection system and subsequently ignores the alarms $(99,100)$.

Further implementation of automated seizure detection is currently hindered by two major issues: EEG recording quality and the presence of periodic epileptiform EEG activity. The neuro-ICU environment creates substantial challenges for the recording of high-quality cEEG accurately reflecting patient's brain activity. One of the greatest difficulties is to maintain a sufficiently low-impedance, high-quality electrode connection between patient and EEG recording machine. This requires a dedicated team of well-trained EEG technicians and nursing staff (4) continuously monitoring signal quality and interacting with the seizure detection algorithm when necessary. Chapter 4 and 5 show that the presence of periodic activity, whether or not epileptiform, may hinder reliable seizure detection. These periodic patterns can occur spontaneously due to pathology or can be elicited by alerting stimuli such as patient handling which have been seen in about $22 \%$ of critically ill patients [8]. Simultaneous video recording during $C E E G$ and documenting patient stimulation effects are necessary to optimize cEEG monitoring. This is especially important for the patient-specific 


\section{Chapter 7}

detection strategy as proposed in chapter 5 . For these reasons, all people involved in neuro-monitoring, from EEG technician to nurse and to clinical neurophysiologist should work closely together to optimize EEG recording quality and automated seizure detection (4). A more fundamental and challenging issue is the distinction between actual seizures and periodic epileptiform activity, a distinction that cannot always be made even by experienced clinical neurophysiologists (73, 101).

\section{Clinical application of automated asymmetry detection during Wada testing.}

During a WADA test it is crucial for reliable assessment of memory and language lateralization that the initiation, termination, and degree of hemispheric inactivation are known. Visual assessment of ipsilateral EEG slowing is the most common monitoring technique used to assess hemispheric inactivation. We showed that QEEG can accurately monitor hemispheric inactivation during Wada tests and can therefore be used to decrease subjectivity of visual EEG judgement. We optimized QEEG based hemispheric inactivation detection by combining temporal and spatial EEG changes in specific brain regions. More specifically, frontal left-right differences $(0.5-12 \mathrm{~Hz}$, channels F3 and F4), and central delta power $(0.5-2.3 \mathrm{~Hz}$, channel $\mathrm{Cz}$ ) relative to a baseline segment prior to amobarbital injection. Our analysis of both the left-right differences and temporal differences separately for each channel and frequency band showed that the BSI response is most pronounced in the delta frequency range which agrees with the fact that visual EEG inspection focuses on ipsilateral delta slowing. However, the duration of hemispheric inactivation based on delta slowing alone would be shorter compared to visual inspection but is compensated by an increased asymmetry in the alpha range $(8-12 \mathrm{~Hz})$ near the end of the annotated period of hemispheric inactivation. This suggests that the information a clinical neurophysiologist uses to determine the termination of 
hemispheric inactivation is more subtle then ipsilateral delta slowing alone. In short, a QEEG measure optimized to best agree with visual EEG judgement might be used to decrease the subjectivity of this visual judgement. It furthermore provides the clinical neurophysiologist with feedback about what his or her visual judgement translates to when expressed in terms of QEEG features. This, if necessary, can then be used to change the set of rules used by a clinical neurophysiologist for assessment of the duration of hemispheric inactivation during Wada testing. This seems contradictory to the definition of visual inspection being the gold standard; it is not in our opinion because also a gold standard should be adaptable if new information becomes available. However, further research is needed, especially investigating interobserver agreement and other objective measures of the effect of amobarbital, to claim that the gold-standard should be revised. This is especially the case in our study because the gold standard was based on the visual judgement of a single clinical neurophysiologist.

\section{Study Limitations}

A limitation of our study is the relatively low number of patients included, and subsequently the number and diversity of seizure and non-seizure patterns in our dataset. Reasons for this limited number of patients are practical ones. First of all, from all patients having a routine EEG, the number of patients who actually have seizures during a routine EEG recording is relatively low and not all of these registrations are tagged as containing seizure activity. Furthermore, the process of providing seizure annotations by an experienced EEG expert is, just like manual EEG monitoring, very time consuming.

Another limitation of our study was the absence of separate annotations for artifacts and epileptiform activity such as GPD. Such annotations would have allowed a quantitative and qualitative evaluation of false detections (50). The reason this evaluation was not performed, was due to the sheer size of our IC EEG dataset and the 


\section{Chapter 7}

limited availability of experienced EEG experts. For the same reason, no per-channel seizure annotations were provided which would have allowed a more sophisticated patient specific SVM update scheme. Nevertheless, from the seizure detection results of our IC EEG dataset, it became clear that when either automated baseline update or patient specific SVM update resulted in worse performance, informal investigation revealed that this was largely due to EEG recording artifacts and periodic epileptiform activity.

\section{Future directions}

As described in chapter 3 , the composition of the training dataset used for classifier training matters for the eventual classifier performance, especially in case of neonatal seizure detection. We did not further investigate further ways of optimizing the training dataset. For example, making a distinction between different seizure types could shed more light on why some seizures are easily detected and why others are not. To optimize a training dataset, a test dataset should be composed that best reflects the diversity of all possible seizure and non-seizure EEG patterns. This should be done in such a way that common EEG patterns do not dominate the test set such that bad performance for uncommon EEG patterns is compensated for by good performance for common EEG patterns. The same reasoning applies to the training dataset as well. An ideal training dataset should be composed of all possible EEG patterns, also in a way that uncommon EEG patterns are outnumbered by common EEG patterns. To achieve this, a method is needed to evaluate how similar a new epoch is to the epochs currently in the dataset. If an epoch is not similar or only similar to a limited amount of other epochs in the current set it is likely to add useful information. On the other hand an epoch very similar to many others would not contribute new information and could even result in the outnumbering of the more uncommon epochs in the data set. In the field of pattern recognition, plenty of algorithms are available to perform such a task. If an algorithm 
to optimize a training dataset is available, it can be used to continuously update the training dataset when new data becomes available, e.g. when a new EEG recording is made or when a database from another institution becomes available as a result of cooperation between institutions. This stresses the importance of cooperation between hospitals and other institutions to develop one common optimized seizure detection algorithm instead of each center developing their own algorithms.

\section{Periodic discharges}

Further research should also be done on the development of algorithms to better distinguish 'seizures' from 'periodic discharges'. This implies that seizure detection is a multi-class problem considering the presence of periodic epileptiform activity which could be seen as an intermediate state on the ictal-interictal continuum. A possibility is to define 3 classes of EEG patterns: non-seizure, interictal, and seizure activity. Our novelty detection approach in chapter 4 can be seen as an attempt to get around this third class, by preventing data from negatively influencing the feature baseline correction. Recognizing interictal activity as a third class also adds useful clinical information because these EEG patterns are associated with a higher risk of seizures $(13,16)$, further neurological damage and subsequent worse outcome [14].However, for example generalized periodic discharges were, contrary to generalized seizures, not independently associated with worse outcome $(14,15$, 102). 
Chapter 7

\section{Conclusions}

- Seizure detection performance can be significantly improved by correcting for differences between baseline EEG feature values between patients as well as for a single patient during a monitoring session.

- During development of a seizure detection algorithm, special attention should be devoted to the training data set composition

- Novelty detection identifies EEG patterns that are different from the current 'normal' non-seizure EEG pattern, and allows for more robust dynamic feature baseline correction.

- By updating the SVM classifier with both good and false detections, seizure detection can be tailored to the individual patient being monitored.

- Asymmetry detection during Wada tests can best be automated by combining left-right hemispheric differences in the frontal regions with temporal changes in delta power in the central regions.

- Evaluation of classification performance is complicated considering the huge effort needed to create a dataset with all relevant annotations as well as the uncertainty of whether a certain pattern is a seizure or not. 
Chapter 8

Valorization 


\section{Chapter 8}

Goal of this thesis is to develop and improve automated epileptic seizure detection algorithms. This goal is a part of a bigger goal, namely to enable reliable EEG neuro-monitoring on the intensive care unit. Our research is most relevant for patient admitted to an ICU unit which can be treated more adequately. There is a general consensus that it is important to be able to detect (non-convulsive) epileptic seizures in comatose ICU patients. In the most optimistic situation timely detection and subsequent intervention with anti-epileptica will lead to the ultimate goal of improved quality of life by preventing further brain damage. Furthermore adequate EEG monitoring could also lead to reduced time spend on the ICU and earlier release from the hospital. Via several ways EEG monitoring can lead to cost reductions. Manual EEG inspection by an EEG expert is very time consuming and therefore costly. ICU stay is expensive, therefore reducing the time spend on the ICU is another way of saving money and resources. Finally an improved quality of life after release from hospital will also result in less spending on for example revalidation and home help.

Although the general consensus is that detecting and treating nonconvulsive seizures in comatose patients is important no consensus exists about whether the use of anti-epileptica in comatose patients result in a better outcome or that it is just etiology that both causes epileptic seizures and determines outcome. Therefore our seizure detection algorithm is also a useful tool for researchers in the field of neuro-monitoring .

Whether used for actual ICU monitoring or as a tool for monitoring studies, our algorithm has to be implemented either as a module in an EEG analysis software package, or as a separate plug-in. Before this final implementation phase can be started first some further research is required. Chapter 3 already indicated that classifier performance depends on the dataset used for training. Furthermore in the different datasets used in this thesis there are some EEG registrations in which the seizures cannot be detected using our current state of the art 
classifier. Upon examination of these EEG registrations it turns out that either the seizures themselves or the specific combination of seizure and non-seizure EEG patterns are not present in the training dataset. In principle a seizure can only be recognized as such by a classifier when similar seizures were 1) present in the training dataset and 2) successfully learned during training. The first point is quite straightforward while the second point can occur when a certain seizure pattern is under represented. It is therefore important to investigate how an optimal training dataset should be constructed. To be able to this a large high quality EEG database is needed. Considering the fact that data availability is often limited and the task of providing annotations is very time consuming, having a high quality training dataset would have great (economical) value. Our automated seizure detection algorithm could also be used to facilitate the construction of such a dataset. As a high quality training dataset is available and the existing methods are optimized final implementation of the seizure detection method should take place. In our opinion the best way to approach this is to cooperate with an existing manufacturer of EEG recording hardware and associated analysis software. In this way there is limited risk compared to starting a company and try to develop and sell it as a software package. Incorporating our seizure detection method into an existing EEG analysis package does not require much effort other than translating the Matlab code into the preferred programming language. Another option is to package our code into a Matlab toolbox which can communicate with an existing package such as BrainRT. This EEG analysis software package already allows for communication with custom Matlab programs. For research purposes the Matlab toolbox can also be published as an open-source toolbox for example as part of the EEGlab toolbox.

The first option would be better when seizure detection is to be used for ICU monitoring while the second option be better when our methods are to be shared in the research community. 

References

\section{References}

1. Boylan GB, Stevenson NJ, Vanhatalo S. Monitoring neonatal seizures. Seminars in Fetal and Neonatal Medicine. 2013;18(4):202-8.

2. DeLorenzo RJ, Waterhouse EJ, Towne AR, Boggs JG, Ko D, DeLorenzo GA, et al. Persistent nonconvulsive status epilepticus after the control of convulsive status epilepticus. Epilepsia. 1998;39(8):833-40.

3. Haas L. Hans Berger (1873-1941), Richard Caton (1842-1926), and electroencephalography. Journal of Neurology, Neurosurgery, and Psychiatry. 2003;74(1):9-.

4. Niedermeyer E, da Silva FL. Electroencephalography: Basic Principles, Clinical Applications, and Related Fields2004.

5. Ebersole JS, Pedley TA. Current practice of clinical electroencephalography, 3rd edn. European Journal of Neurology. 2003;10(5):604-5.

6. Kennedy J, Gerard E. Continuous EEG Monitoring in the Intensive Care Unit. Current Neurology and Neuroscience Reports. 2012:1-10.

7. Peters JM, Tomas-Fernandez $\mathrm{M}$, van Putten MJAM, Loddenkemper $\mathrm{T}$. Behavioral measures and EEG monitoring using the Brain Symmetry Index during the Wada test in children. Epilepsy \& Behavior. 2012;23(3):247-53.

8. Mishra M, Banday M, Derakhshani R, Croom J, Camarata P. A Quantitative EEG Method for Detecting Post Clamp Changes During Carotid Endarterectomy. J Clin Monit Comput. 2011;25(5):295-308.

9. Jasper $\mathrm{HH}$. Report of the committee on methods of clinical examination in electroencephalography: 1957. Electroencephalography and Clinical Neurophysiology. 1958;10(2):370-5.

10. Malmivuo J, Plonsey R. Bioelectromagnetism1995.

11. Jeong J. EEG dynamics in patients with Alzheimer's disease. Clinical Neurophysiology. 2004;115(7):1490-505.

12. Holmes G. Childhood-specific epilepsies accompanied by developmental disabilities: causes and effects. Epilepsy and Developmental Disabilities. 2001.

13. Lawrence J. Hirsch RPB. Atlas of EEG in Critical Care2010.

14. Akman $\mathrm{Cl}$, Riviello JJ, Jr. Generalized periodic epileptiform discharges in critically ill children: a continuum of status epilepticus or an epiphenomenon? Journal of clinical neurophysiology : official publication of the American Electroencephalographic Society. 2011;28(4):366-72.

15. Akman Cl, Abou Khaled KJ, Segal E, Micic V, Riviello JJ. Generalized periodic epileptiform discharges in critically ill children: clinical features, and outcome. Epilepsy Res. 2013;106(3):378-85. 


\section{References}

16. Chong DJ, Hirsch LJ. Which EEG patterns warrant treatment in the critically ill? Reviewing the evidence for treatment of periodic epileptiform discharges and related patterns. Journal of clinical neurophysiology : official publication of the American Electroencephalographic Society. 2005;22(2):79-91.

17. Vanhatalo S, Voipio J, Kaila K. Full-band EEG (FbEEG): an emerging standard in electroencephalography. Clinical Neurophysiology. 2005;116(1):1-8.

18. Sackellares JC, Shiau D-S, Halford JJ, LaRoche SM, Kelly KM. Quantitative EEG analysis for automated detection of nonconvulsive seizures in intensive care units. Epilepsy and Behavior. 2011;22, Supplement 1(0):S69-S73.

19. Lim AJ, Winters WD. A Practical Method for Automatic Real-Time EEG Sleep State Analysis. Biomedical Engineering, IEEE Transactions on. 1980;BME27(4):212-20.

20. Zhang XS, Roy RJ, Jensen EW. EEG complexity as a measure of depth of anesthesia for patients. IEEE Transactions on Biomedical Engineering. 2001;48(12):1424-33.

21. Shin HC, Tong S, Yamashita S, Jia X, Geocadin RG, Thakor NV. Quantitative EEG assessment of brain injury and hypothermic neuroprotection after cardiac arrest. Conference Proceedings, Annual International Conference of the IEEE Engineering in Medicine and Biology Society. 2006;1:6229-32.

22. Latif MA, Sanei S, Chambers J, Shoker L. Localization of abnormal EEG sources using blind source separation partially constrained by the locations of known sources. Signal Processing Letters, IEEE. 2006;13(3):117-20.

23. Bouwer MS, Jones-Gotman M, Gotman J. Duration of sodium amytal effect: behavioral and EEG measures. Epilepsia. 1993;34(1):61-8.

24. Whittemore AD, Kauffman JL, Kohler TR, Mannick JA. Routine electroencephalographic (EEG) monitoring during carotid endarterectomy. Annals of Surgery. 1983;197(6):707-13.

25. Towne AR, Waterhouse EJ, Boggs JG, Garnett LK, Brown AJ, Smith JR, Jr., et al. Prevalence of nonconvulsive status epilepticus in comatose patients. Neurology. 2000;54(2):340-5.

26. Claassen J, Mayer SA, Kowalski RG, Emerson RG, Hirsch LJ. Detection of electrographic seizures with continuous EEG monitoring in critically ill patients. Neurology. 2004;62(10):1743-8.

27. Husain AM, Horn GJ, Jacobson MP. Non-convulsive status epilepticus: usefulness of clinical features in selecting patients for urgent EEG. Journal of Neurology, Neurosurgery and Psychiatry. 2003;74(2):189-91. 
28. Drislane FW, Lopez MR, Blum AS, Schomer DL. Detection and treatment of refractory status epilepticus in the intensive care unit. Journal of Clinical Neurophysiology. 2008;25(4):181-6.

29. Pandian JD, Cascino GD, So EL, Manno E, Fulgham JR. Digital videoelectroencephalographic monitoring in the neurological-neurosurgical intensive care unit: clinical features and outcome. Archives of Neurology. 2004;61(7):10904.

30. Narayanan JT, Murthy JM. Nonconvulsive status epilepticus in a neurological intensive care unit: profile in a developing country. Epilepsia. 2007;48(5):900-6.

31. Tong S, Thakor NV. Quantitative EEG Analysis Methods and Clinical Applications. Yarmush ML, J. James C, editors. Norwood: ARTECH HOUSE; 2009.

32. Agarwal R, Gotman J, Flanagan D, Rosenblatt B. Automatic EEG analysis during long-term monitoring in the ICU. Electroencephalography and Clinical Neurophysiology. 1998;107(1):44-58.

33. Borel C, Hanley D. Neurologic intensive care unit monitoring. Critical Care Clinics. 1985;1(2):223-39.

34. Greene BR, Faul S, Marnane WP, Lightbody G, Korotchikova I, Boylan GB. A comparison of quantitative EEG features for neonatal seizure detection. Clinical Neurophysiology. 2008;119(6):1248-61.

35. Temko A, Nadeu C, Marnane W, Boylan GB, Lightbody G. EEG Signal Description with Spectral-Envelope-Based Speech Recognition Features for Detection of Neonatal Seizures. Information Technology in Biomedicine, IEEE Transactions on. 2011;15(6):839-47.

36. Pachori RB, Bajaj V. Analysis of normal and epileptic seizure EEG signals using empirical mode decomposition. Computer Methods and Programs in Biomedicine. 2011;104(3):373-81.

37. McLachlan G. Discriminant Analysis and Statistical Pattern Recognition (Wiley Series in Probability and Statistics): Wiley-Interscience; 2004.

38. Cortes C, Vapnik V. Support-vector networks. Mach Learn. 1995;20(3):27397.

39. Scholkopf B SA. Learning with kernels: MIT press; 2002.

40. Platt. Probabilistic outputs for SVM and comparisons to regularized likelihood methods. 1999.

41. A Fawcett T. ROC graphs: Notes and practical considerations for researchers. Technical Report HPL-2003-4. 2004.

42. Temko A, Thomas E, Marnane W, Lightbody G, Boylan G. EEG-based neonatal seizure detection with Support Vector Machines. Clinical Neurophysiology. 2011;122(3):464-73. 


\section{References}

43. van Putten MJ, Peters JM, Mulder SM, de Haas JA, Bruijninckx CM, Tavy DL. A brain symmetry index (BSI) for online EEG monitoring in carotid endarterectomy. Clinical neurophysiology : official journal of the International Federation of Clinical Neurophysiology. 2004;115(5):1189-94.

44. Wirrell EC, Armstrong EA, Osman LD, Yager JY. Prolonged seizures exacerbate perinatal hypoxic-ischemic brain damage. Pediatric Research. 2001;50(4):445-54.

45. Gotman J, Flanagan D, Zhang J, Rosenblatt B. Automatic seizure detection in the newborn: methods and initial evaluation. Electroencephalography and Clinical Neurophysiology. 1997;103(3):356-62.

46. Navakatikyan MA, Colditz PB, Burke CJ, Inder TE, Richmond J, Williams CE. Seizure detection algorithm for neonates based on wave-sequence analysis. Clinical Neurophysiology. 2006;117(6):1190-203.

47. Aarabi A, Grebe R, Wallois F. A multistage knowledge-based system for EEG seizure detection in newborn infants. Clinical Neurophysiology. 2007;118(12):2781-97.

48. Aarabi A, Wallois F, Grebe R. Automated neonatal seizure detection: A multistage classification system through feature selection based on relevance and redundancy analysis. Clinical Neurophysiology. 2006;117(2):328-40.

49. Deburchgraeve W, Cherian PJ, De Vos M, Swarte RM, Blok JH, Visser GH, et al. Automated neonatal seizure detection mimicking a human observer reading EEG. Clinical Neurophysiology. 2008;119(11):2447-54.

50. Temko A, Thomas E, Marnane W, Lightbody G, Boylan GB. Performance assessment for EEG-based neonatal seizure detectors. Clinical Neurophysiology. 2011;122(3):474-82.

51. Tharp BR. Electrophysiological brain maturation in premature infants: an historical perspective. Journal of Clinical Neurophysiology. 1990;7(3):302-14.

52. Scher MS, Steppe DA, Sclabassi RJ, Banks DL. Regional differences in spectral EEG measures between healthy term and preterm infants. Pediatric Neurology. 1997;17(3):218-23.

53. Chisci L, Mavino A, Perferi G, Sciandrone M, Anile C, Colicchio G, et al. RealTime Epileptic Seizure Prediction Using AR Models and Support Vector Machines. IEEE Transactions on Biomedical Engineering. 2010;57(5):1124-32.

54. De Weerd AW, Despland PA, Plouin P. Neonatal EEG. The International Federation of Clinical Neurophysiology. Electroencephalography and Clinical Neurophysiology. 1999;52:149-57.

55. Vapnik. Estimation of Dependences Based on Empirical Data: Springer Series in Statistics (Springer Series in Statistics): Springer-Verlag New York, Inc.; 1982. 
56. Rauch HE, Tung F, Striebel CT. Maximum likelihood estimates of linear dynamic systems. AIAA Journal. 1965;3(8):1445-50.

57. MATLAB. version 7.14.0. (R2012a). Natick, Massachusetts: The MathWorks Inc.; 2012.

58. Hanley JA, McNeil BJ. A method of comparing the areas under receiver operating characteristic curves derived from the same cases. Radiology. 1983;148(3):839-43.

59. Scher MS, Hamid MY, Steppe DA, Beggarly ME, Painter MJ. Ictal and interictal electrographic seizure durations in preterm and term neonates. Epilepsia. 1993;34(2):284-8.

60. Bar-Shalom Y, Kirubarajan T, Li X-R. Estimation with Applications to Tracking and Navigation: John Wiley \& Sons, Inc.; 2002.

61. Scheuer ML. Continuous EEG monitoring in the intensive care unit. Epilepsia. 2002;3:114-27.

62. Privitera $M$, Hoffman $M$, Moore JL, Jester D. EEG detection of nontonicclonic status epilepticus in patients with altered consciousness. Epilepsy Research. 1994;18(2):155-66.

63. Ramgopal S, Thome-Souza S, Jackson M, Kadish NE, Sánchez Fernández I, Klehm J, et al. Seizure detection, seizure prediction, and closed-loop warning systems in epilepsy. Epilepsy and Behavior. 2014;37(0):291-307.

64. Hartmann MM, Furbass F, Perko H, Skupch A, Lackmayer K, Baumgartner C, et al., editors. EpiScan: Online seizure detection for epilepsy monitoring units. Engineering in Medicine and Biology Society, EMBC, 2011 Annual International Conference of the IEEE; 2011 Aug. 30 2011-Sept. 32011.

65. Logesparan L, Rodriguez-Villegas E, Casson AJ. The impact of signal normalization on seizure detection using line length features. Medical \& biological engineering \& computing. 2015.

66. Bogaarts JG, Gommer ED, Hilkman DMW, van Kranen-Mastenbroek VHJM, Reulen JPH. EEG Feature Pre-processing for Neonatal Epileptic Seizure Detection. Ann Biomed Eng. 2014:1-9.

67. Duun-Henriksen J, Kjaer TW, Madsen RE, Remvig LS, Thomsen CE, Sorensen HBD. Channel selection for automatic seizure detection. Clinical Neurophysiology.In Press, Corrected Proof.

68. Pimentel MAF, Clifton DA, Clifton L, Tarassenko L. A review of novelty detection. Signal Processing. 2014;99(0):215-49.

69. Hoffmann H. Kernel PCA for novelty detection. Pattern Recognition. 2007;40(3):863-74.

70. Jolliffe IT. Principal Component Analysis: Springer Verlag; 1986. 


\section{References}

71. Faul S, Temko A, Marnane W. Age-independent seizure detection. Conference Proceedings, Annual International Conference of the IEEE Engineering in Medicine and Biology Society. 2009;5(10):5332553.

72. Sierra-Marcos A, Scheuer ML, Rossetti AO. Seizure detection with automated EEG analysis: A validation study focusing on periodic patterns. Clinical Neurophysiology. 2015;126(3):456-62.

73. Ronner HE, Ponten SC, Stam CJ, Uitdehaag BMJ. Inter-observer variability of the EEG diagnosis of seizures in comatose patients. Seizure. 2009;18(4):257-63.

74. Haas SM, Frei MG, Osorio I. Strategies for adapting automated seizure detection algorithms. Medical engineering \& physics. 2007;29(8):895-909.

75. Shoeb A, Bourgeois B, Treves S, Schachter SC, Guttag J. Impact of patientspecificity on seizure onset detection performance. Conference proceedings : Annual International Conference of the IEEE Engineering in Medicine and Biology Society IEEE Engineering in Medicine and Biology Society Annual Conference. 2007;2007:4110-4.

76. Thomas EM, Greene BR, Lightbody G, Marnane WP, Boylan GB, editors. Seizure detection in neonates: Improved classification through supervised adaptation. Engineering in Medicine and Biology Society, 2008 EMBS 2008 30th Annual International Conference of the IEEE; 2008 20-25 Aug. 2008.

77. Wada J, Rasmussen T. Intracarotid injection of sodium amytal for the lateralization of cerebral speech dominance. 1960. Journal of neurosurgery. 1960;106(6):1117-33.

78. Buchtel HA, Passaro EA, Selwa LM, Deveikis J, Gomez-Hassan D. Sodium methohexital (brevital) as an anesthetic in the Wada test. Epilepsia. 2002;43(9):1056-61.

79. Magee JA, Pender NP, Abrahams S, Thornton J, Delanty N, Fortune GM. A comparison of propofol and amobarbital for use in the Wada test. Seizure. 2012;21(5):399-401.

80. Patel A, Wordell C, Szarlej D. Alternatives to sodium amobarbital in the Wada test. The Annals of pharmacotherapy. 2011;45(3):395-401.

81. Blume WT, Grabow JD, Darley FL, Aronson AE. Intracarotid amobarbital test of language and memory before temporal lobectomy for seizure control. Neurology. 1973;23(8):812-9.

82. Acharya JN, Dinner DS. Use of the intracarotid amobarbital procedure in the evaluation of memory. Journal of clinical neurophysiology : official publication of the American Electroencephalographic Society. 1997;14(4):311-25.

83. Williams GW, Luders HO, Brickner A, Goormastic M, Klass DW. Interobserver variability in EEG interpretation. Neurology. 1985;35(12):1714-9. 
84. Sharan A, Ooi YC, Langfitt J, Sperling MR. Intracarotid amobarbital procedure for epilepsy surgery. Epilepsy \& behavior : E\&B. 2011;20(2):209-13.

85. Roberts DW. Is there still a role for language-Wada testing? World neurosurgery. 2011;75(3-4):425-7.

86. Baxendale S. The Wada test. Current opinion in neurology. 2009;22(2):1859.

87. Tu B, Assassi NJ, Bazil CW, Hamberger MJ, Hirsch LJ. Quantitative EEG is an objective, sensitive, and reliable indicator of transient anesthetic effects during Wada tests. Journal of clinical neurophysiology : official publication of the American Electroencephalographic Society. 2015;32(2):152-8.

88. van Putten MJ. The revised brain symmetry index. Clin Neurophysiology. 2007;118(11):2362-7.

89. van Putten MJ, Tavy DL. Continuous quantitative EEG monitoring in hemispheric stroke patients using the brain symmetry index. Stroke. 2004;35(11):2489-92.

90. Kalman RE. A New Approach to Linear Filtering and Prediction Problems. Journal of Fluids Engineering. 1960;82(1):35-45.

91. Jones-Gotman M, Bouwer MS, Gotman J. EEG slow waves and memory performance during the intracarotid amobarbital test. Epilepsia. 1994;35(1):61-9.

92. Ahern GL, Labiner DM, Talwar D, Herring AM, Weinand ME. Quantitative analysis of the electroencephalogram in the intracarotid amobarbital procedure: II. Coherence analysis. Journal of clinical neurophysiology : official publication of the American Electroencephalographic Society. 1995;12(3):285-90.

93. Hong SB, Kim KW, Seo DW, Kim SE, Na DG, Byun HS. Contralateral EEG slowing and amobarbital distribution in Wada test: an intracarotid SPECT study. Epilepsia. 2000;41(2):207-12.

94. Gotman J, Bouwer MS, Jones-Gotman M. Intracranial EEG study of brain structures affected by internal carotid injection of amobarbital. Neurology. 1992;42(11):2136-43.

95. Douw L, Baayen JC, Klein M, Velis D, Alpherts WC, Bot J, et al. Functional connectivity in the brain before and during intra-arterial amobarbital injection (Wada test). Neurolmage. 2009;46(3):584-8.

96. Temko A, Stevenson N, Marnane W, Boylan G, Lightbody G. Inclusion of temporal priors for automated neonatal EEG classification. Journal of neural engineering. 2012;9(4):046002.

97. Temko A, Lightbody G, Thomas EM, Boylan GB, Marnane W. Instantaneous measure of EEG channel importance for improved patient-adaptive neonatal 


\section{References}

seizure detection. IEEE transactions on bio-medical engineering. 2012;59(3):71727.

98. Tang Y, Durand DM. A tunable support vector machine assembly classifier for epileptic seizure detection. Expert Systems with Applications. 2012;39(4):392538.

99. Sowan AK, Gomez TM, Tarriela AF, Reed CC, Paper BM. Changes in Default Alarm Settings and Standard In-Service are Insufficient to Improve Alarm Fatigue in an Intensive Care Unit: A Pilot Project. JMIR human factors. 2016;3(1):e1.

100. Sendelbach $S$, Funk M. Alarm fatigue: a patient safety concern. AACN advanced critical care. 2013;24(4):378-86; quiz 87-8.

101. Hirsch LJ, LaRoche SM, Gaspard N, Gerard E, Svoronos A, Herman ST, et al. American Clinical Neurophysiology Society's Standardized Critical Care EEG Terminology: 2012 version. Journal of clinical neurophysiology : official publication of the American Electroencephalographic Society. 2013;30(1):1-27.

102. Foreman B, Claassen J, Abou Khaled K, Jirsch J, Alschuler DM, Wittman J, et al. Generalized periodic discharges in the critically ill: a case-control study of 200 patients. Neurology. 2012;79(19):1951-60. 
Summary 
Summary

\section{Summary}

Brain damage results in many types of brain dysfunction ranging from impairment of specific functions, complete loss of consciousness, to the occurrence of epileptic seizures. Although continuous EEG (cEEG) allows for real-time monitoring of brain functioning, it is very labour intensive and often retrospective. Development of automated EEG analysis methods is therefore much warranted. Especially automated detection of seizures in the EEG could be a valuable tool to assist the human expert.

In this thesis we used signal analysis and machine learning techniques to improve automated seizure detection algorithms. Based on a large database containing annotated EEG epochs from many patients, these Algorithms 'learn' to detect epochs containing seizure activity. In the second chapter, the seizure detection learning task is simplified by introducing a feature baseline correction (FBC) method that reduces inter-patient variability. This resulted in a significant improvement in seizure detection performance in a cohort of neonatal EEG recordings.

Furthermore, we demonstrate in chapter 3 that FBC allowed for the successful merge of neonatal and adult seizure detection. Before, adultand neonatal- seizure detection were considered as two distinct entities which was proved here to be only partly true. Adult seizure detection could be successfully performed with either a classifier trained on adult or neonatal EEG data. However, neonatal seizure detection performance of a classifier trained on adult EEG data was significantly worse compared to the performance of one trained on neonatal EEG data.

So far, FBC was constant over time and seizure detection with FBC was only evaluated using relatively short ( 20 minutes) EEG recordings for which it can be assumed that the baseline EEG characteristics remain 152 
constant. This is however not the case in long-term EEG recordings that can last up to several days. Therefore, we further improved seizure detection performance on long-term recordings by developing a novelty detection based method that automatically adapts FBC to changes in the EEG characteristics. Although the general effect was positive, in case of periodic epileptiform discharges, that often wax and wane but also turn into seizures, the automatic baseline update could result in a large reduction of false alarms but at the cost of not detecting the seizures as well.

Analysis of seizure detection performance in long-term EEG recordings of comatose patients revealed that the mayor source of false alarms was caused by the before mentioned periodic epileptiform discharges. To enable the detection algorithm to learn from past experience, we simulated a setting where EEG data of both good and false detections was used online to update the classifier. Also this resulted in improved seizure detection performance in general. However, in cases when periodic discharges and seizures were to similar, the end effect was either detecting all periodic discharges and seizures or nothing at all.

The topic of the last chapter is the Wada test, another field where epilepsy and quantitative EEG are combined. The Wada test is commonly used to evaluate language and memory lateralisation in epilepsy surgery candidates. EEG can be used to assist in determining the duration of hemispheric deactivation after Amobarbital injection. We develop a quantitative EEG measure that optimally matches the duration of hemispheric deactivation as indicated by a clinical neurophysiologist. This resulted in the combination of a measure of spatial asymmetry in the frontal cerebral regions with a measure of temporal changes in the activity in the central regions. 

Samenvatting 
Samenvatting

\section{Samenvatting}

Schade aan het brein kan resulteren in verschillende soorten van dysfunctioneren zoals een verslechtering van cognitieve functies, het verliezen van bewustzijn en het hebben van epileptische aanvallen. Ondanks dat continue EEG registratie het mogelijk maakt om het functioneren van het brein in real-time te monitoren, is het nog steeds erg arbeids intensief en vaak retrospectief. Het ontwikkelen van automatische EEG analyse methoden is daarom gegrond. Met name het automatisch detecteren van epileptische aanvallen zou een waardevol hulpmiddel zijn om een EEG expert te ondersteunen.

In dit proefschrift hebben we gebruik gemaakt van signaal analyse en machine learning technieken om algoritme voor automatisch detectie van epileptische aanvallen te verbeteren. Gebruikmakend van een database met geannoteerde EEGs van verschillende patienten, leert een algoritme hoe aanvals van niet-aanvals epochen onderscheiden kunnen worden. In het tweede hoofdstuk introduceren we een 'feature baseline' correctie (FBC) methode die de leeropdracht voor het algoritme vereenvoudigd door een deel van de verschillen tussen patienten te elimineren. Dit resulteerde in een verbetering van aanvals detectie acuraatheid bij neonatale EEG registraties.

Vervolgens laten we in hoofdstuk 3 zien dat FBC het ook mogelijk maakt om aanvals detectie bij neonaten en volwassenen te combineren. Voorheen werden aanvals detectie bij neonaten en volwassenen gezien als twee aparte entiteiten wat maar gedeeltelijk zo blijkt te zijn. Aanvals detectie bij volwassenen kan succesvol gedaan worden met een algoritme dat getraind is op EEG van neonaten danwel volwassenen. Echter, voor aanvals detectie bij neonaten bleek dat een algoritme getraind met EEG van volwassenen significant slechter presteerde dan een algoritme getraind met neonataal EEG. 
Tot nu was FBC statisch en aanvals detectie met FBC slechts geevalueerd met relatief korte ( $\sim 20$ minuten) EEG registraties waarvan kan worden aangenomen dat de EEG karakteristieken min of meer constant blijven. Dit is echter niet het geval bij EEG registraties die tot enkele dagen lang zijn. Daarom hebben we het aanvals detectie algoritme verbeterd door een methode, gebaseerd op 'novelty detectie', te intoduceren. Met deze methode wordt het algoritme automatisch aanpast aan veranderingen in EEG eigenschappen. In het algemeen resulteerde dit in een reductie van valse alarmen. Echter, in het geval van periodieke ontladingen, die vaak fluctuerend in hevigheid voorkomen maar ook vaak uitgroeien tot een aanval, resulteerde het automatisch aanpassen van FBC erin dat er uberhaupt niets meer gedetecteerd werd.

Het analyseren van het functioneren van de aanvals detectie bij langdurige EEG registraties bij comateuze patienten maakte duidelijk dat de periodieke ontladingen de grootste bron van valse alarmen waren. Om het mogelijk te maken dat een algoritme leert van de in het verleden opgedane ervaring hebben we een situatie gesimuleerd waarbij zowel goede als valse detecties gebruikt werden om het algoritme te updaten. Dit resulteerde ook in verbeterde prestaties, echter, net als bij de automatische FBC, resulteerde dit vaak juist in slechtere prestaties bij EEGs met zowel periodieke ontladingen en aanvallen. Concreet werden dan ofwel alle aanvallen en periodieke ontladingen gedetecteerd of helemaal niets.

Het onderwerp van het laatste hoofdstuk is de Wada test, een ander toepassing van EEG op het gebied van epilepsie. De wada test wordt gebruikt om bij epilepsie patienten die in aanmerking komen voor een operatie, te evalueren aan welke kant van het brein spraak en geheugen zijn gelokaliseerd. EEG kan worden gebruikt om te assisteren bij het bepalen van de duur van hemispherische deactivatie na het injecteren van Amobarbital. We hebben een kwantitatieve EEG maat ontwikkeld 
Samenvatting

die de duur van deactivatie, zoals aangegeven door een klinisch neurphysioloog, zo goed mogelijk reproduceert. Deze maat combineert spatiele asymmetry in de frontale regionen met temporele veranderingen in de centrale brein regionen. 
Dankwoord 
Dankwoord

Dankwoord

Nu mijn proefschrift zo goed als af is moet alleen nog het dankwoord geschreven worden. Over wat ik precies zou moeten schrijven heb ik natuurlijk nog nauwelijks nagedacht. Gelukkig heb ik het proefschrift van Erik als voorbeeld en is de plagiaat controle toch al achter de rug. Terugblikkend op mijn promotietraject kan ook ik concluderen dat afzien een treffende omschrijving is. Niet zozeer datgene wat ik versta onder het echte werk en de zeer prettige samenwerking met mijn collega's, als wel het schrijfwerk en alle administratieve bijkomstigheden. Ik ben dan ook een aantal mensen zeer dankbaar die er voor hebben gezorgd dat ik uberhaupt dit traject heb doorlopen en het uiteindelijk ook succesvol heb kunnen afronden.

Prof. dr. Mess, beste Werner, ik will jou bedanken voor de mogelijkheid om te promoveren binnen jouw afdeling. Het geven van vertrouwen heeft er voor gezorgd dat ik in relatieve rust en vrijheid samen met Jos en Erik onderzoek heb kunnen doen.

Dr. ir. Reulen, beste Jos, na het afronden van mijn master project bij jou en Erik was ik ineens student af en moest ineens het grote mensen leven beginnen, iets waar ik totaal niet op voorbereid was. Wellicht in een poging om de realiteit te ontwijken heb ik me toen volledig gestort op het wielrennen, iets dat achteraf natuurlijk erg onrealistisch was. Gelukkig was jij mij niet vergeten en hebt toen na een paar maanden gevraagd of ik geinteresseerd was in het doen van een PhD. Ondanks de af en toe ongezoute kritiek heb ik onze samenwerking die gebaseerd was op wederzijds respect als erg prettig ervaren.

Dr. ir. Gommer, beste Erik, met jouw ideeen, feedback als ook het fungeren als klankbord hebben hebben een belangrijke bijdrage aan dit proefschrift geleverd. Wat onze samenwerking nog leuker heeft gemaakt was onze gedeelde passie: Wielrennen. Het bespreken van de 
koers van het afgelopen weekend, het delen van de ontdekking van alweer een klein weggetje in de Voerstreek, en het vertellen over onze eigen fiets avonturen is nu helaas gereduceerd tot het geven van kudos op Strava. Eigenlijk is het vreemd dat we nooit samen gefietst hebben dus wil ik je bij deze uitnodigen om in Basel een mooie rit te maken!

Prof.dr. H.W.M. Steinbusch, Prof.dr. ir. S.J.A. van Huffel, Prof.dr. ir. M.J.A.M. van Putten, Dr. W.N.K.A. van Mook, Dr. M.C.G. Vlooswijk, bedankt voor jullie inspanningen bij het beoordelen van mijn proefschrift.

Danny en Vivianne, bedankt dat ik altijd bij jullie heb kunnen binnen lopen met vragen, door jullie heb ik nu ook enigzins ervaring met het 'lezen' van EEGs.

Beste laboranten, hartsikke bedankt voor het annoteren van alle EEGs, niet het leukste werk maar toch essentieel voor mijn onderzoek.

Keir, bedankt voor het verbeteren van grammatica van mijn artikelen. Wanneer plannen we onze fietstoch Basel-Maastricht?

Mijn vrienden, Frank, Daan, Guido, Lodewijk, Martijn, Rene, Vladimir Jorn, Luc, Tom, en Ward. Het contact met jullie is mij erg veel waard! Ik hoop dat ik met iederen in contact blijf en we nog veel mooie dingen kunnen doen.

Lieve Pauke, Roel en Florieke en natuurlijk ook Marc, Jeanine en Timo, nu ik zo ver van jullie vandaan zit hoop ik dat onze hechte band alleen maar sterker wordt. Ik kijk uit naar alle mooie dingen die wij nog samen gaan meemaken! Marc wil ik in het bijzonder bedanken voor het ontwerpen van de kaft zodat mijn boekje er ook uitziet als een echt proefschrift. 
Dankwoord

Mijn ouders: Papa en Mama. In de wetenschap is het willen weten wellicht het belangrijkste. Papa, mijn vroegste herinnering aan het willen weten hoe dingen werken gaan terug naar de tijd dat jij mij naar bed bracht en ik dan mocht vragen hoe iets zoals een cd speler werkt. Mama, vanaf dat ik geboren ben heb jij er alles aan gedaan om er voor te zorgen dat ik mij heb kunnen ontwikkelen tot wie ik nu ben. Van minder goede ideeen zoals dans-expressie tot het kiezen van mijn opleiding Biomedische technologie.

Lieve Milou, mijn promotie periode is grotendeels voor jouw tijd maar ik wil deze mogelijkheid toch aangrijpen om iets te zeggen wat jij eigenlijk toch al weet: $\mathrm{jij}$ bent het mooiste wat mij is overkomen en om samen met jou te zijn is als een droom waaruit ik hopelijk nooit zal ontwaken! 


\section{List of publications}

Gommer ED, Bogaarts G, Martens EG, Mess WH, Reulen JP. Visually evoked blood flow responses and interaction with dynamic cerebral autoregulation: correction for blood pressure variation. Medical engineering \& physics. 2014;36(5):613-9.

Bogaarts JG, Gommer ED, Hilkman DMW, van Kranen-Mastenbroek VHJM, Reulen JPH. EEG Feature Pre-processing for Neonatal Epileptic Seizure Detection. Ann Biomed Eng. 2014:1-9.

Bogaarts JG, Gommer ED, Hilkman DM, van Kranen-Mastenbroek VH, Reulen JP. Optimal training dataset composition for SVM-based, ageindependent, automated epileptic seizure detection. Medical \& biological engineering \& computing. 2016;54(8):1285-93.

Bogaarts JG, Hilkman DM, Gommer ED, van Kranen-Mastenbroek VH, Reulen JP. Improved epileptic seizure detection combining dynamic feature normalization with EEG novelty detection. Medical \& biological engineering \& computing. 2016

Bogaarts G, Gommer E, Hilkman D, van Kranen-Mastenbroek V, Reulen J. An improved qEEG index for asymmetry detection during the Wada test. Epilepsy \& behavior : E\&B. 2016;62:40-6.

Schipper S, Aalbers MW, Rijkers K, Lagiere M, Bogaarts JG, Blokland A, et al. Accelerated cognitive decline in a rodent model for temporal lobe epilepsy. Epilepsy \& behavior : E\&B. 2016;65:33-41. 


\section{Curriculum Vitae}

\section{Curriculum Vitae}

Guy Bogaarts was born on April 2, 1986 in Waalwijk, the Netherlands. He grew up in Geertruidenberg, Waalwijk and Roermond. He finished his secondary education (VWO) in 2005 at the Bisschoppelijk college Broekhin in Roermond. He started his study Biomedical Engineering at Eindhoven University of Technology the where he obtained his Master's degree in Medical Engineering in 2010. In 2011 he started his PhD in epileptic seizure detection at the department of clinical neurophysiology of the Maastricht university hospital. Currently, he is living together with Milou Meeuse in Basel where he works as a postdoctoral researcher at the Universitätsspital. 